INSTITUT NATIONAL DE RECHERCHE EN INFORMATIQUE ET EN AUTOMATIQUE

\title{
Serre's reduction of linear functional systems
}

Mohamed S. Boudellioua and Alban Quadrat

\section{$\mathbf{N}^{\circ} \mathbf{7 2 1 4}$}

February 2010

- Algorithms, Certification, and Cryptography _ 



\title{
Serre's reduction of linear functional systems
}

\author{
Mohamed S. Boudellioua* and Alban Quadrat ${ }^{\dagger}$ \\ Theme : Algorithms, Certification, and Cryptography \\ Équipe-Projet AT-SOP \\ Rapport de recherche $\mathrm{n}^{\circ} 7214$ - February 2010 - 56 pages
}

\begin{abstract}
Serre's reduction aims at reducing the number of unknowns and equations of a linear functional system (e.g., system of partial differential equations, system of differential time-delay equations, system of difference equations). Finding an equivalent representation of a linear functional system containing fewer equations and fewer unknowns generally simplifies the study of its structural properties, its closed-form integration as well as of different numerical analysis issues. The purpose of this paper is to present a constructive approach to Serre's reduction for determined and underdetermined linear functional systems.
\end{abstract}

Key-words: Serre's reduction, linear functional systems, module theory, homological algebra, symbolic computation, mathematical systems theory.

* Mathematics and Statistics Department, Sultan Qaboos University, P. O. Box 36 Al-Khodh, 123, Muscat, Oman, boudell@squ.edu.om.

$\dagger$ INRIA Sophia Antipolis, 2004 Route des Lucioles BP 93, 06902 Sophia Antipolis Cedex, France, Alban.Quadrat@sophia.inria.fr. 


\section{Réduction de Serre des systèmes linéaires fonctionnels}

Résumé : La réduction de Serre a pour but de réduire le nombre d'inconnues et d'équations d'un système linéaire fonctionnel (e.g., système d'équations aux dérivées partielles, système d'équations différentielles à retard, système d'équations aux différences). Trouver une représentation équivalente d'un système linéaire fonctionnel contenant moins d'équations et d'inconnues simplifie généralement l'étude de ses propriétés structurelles, son intégration sous forme fermée ainsi que différents problèmes d'analyse numérique. Le but de ce papier est de présenter une approche constructive de la réduction de Serre des systèmes linéaires fonctionnels déterminés et sous-déterminés.

Mots-clés : Réduction de Serre, systèmes linéaires fonctionnels, théorie des modules, algèbre homologique, calcul formel, théorie mathématique des systèmes. 


\section{Introduction}

Over the years, the Smith normal form has played an important role in the study of linear systems defined over a univariate commutative polynomial ring $k[x]$, where $k$ is a field (e.g., the univariate commutative polynomial ring of ordinary differential operators with coefficients in a differential field of constants). For more details, see, e.g., [17, 37] and the references therein.

The concept of the Smith normal form can be extended to a matrix $R$ with entries in a multivariate commutative polynomial ring $D=k\left[x_{1}, \ldots, x_{n}\right]$ over a field $k$ when defined as being the diagonal matrix $\operatorname{diag}\left(\gamma_{1}, \ldots, \gamma_{r}\right)$ formed by the polynomials $\gamma_{1}, \ldots, \gamma_{r}$ defined as the successive quotients $\gamma_{i}=\alpha_{i} / \alpha_{i-1}$ of the greatest common divisors $\alpha_{i}$ of the $i \times i$-minors of the matrix $R$ ( $\alpha_{0}=1, r$ is the rank of the matrix). Despite its relevance in mathematical systems theory and numerical analysis, the problem of the equivalence of a multivariate polynomial matrix to its Smith normal form by means of unimodular transformations has only been sparsely studied in the mathematical systems theory literature. See a few exceptions [3, 14, 15, 16, 20].

An interesting result in this direction is the following one.

Theorem $1([3,16])$. Let $D=\mathbb{R}\left[x_{1}, \ldots, x_{n}\right]$ be the commutative polynomial ring with coefficients in $\mathbb{R}, R \in D^{p \times p}$ a full row rank matrix, i.e., $\operatorname{det} R \neq 0$, and $\mathbb{R}^{\star}=\mathbb{R} \backslash\{0\}$. Then, there exist two unimodular matrices $V \in D^{p \times p}$ and $W \in D^{p \times p}$, i.e., $\operatorname{det} V \in \mathbb{R}^{\star}$ and $\operatorname{det} W \in \mathbb{R}^{\star}$, such that

$$
V R W=\left(\begin{array}{cc}
I_{p-1} & 0 \\
0 & \operatorname{det} R
\end{array}\right)
$$

iff there exists a column vector $\Lambda \in D^{p}$ admitting a left-inverse over $D$ and such that the matrix $P \triangleq\left(\begin{array}{ll}R & -\Lambda\end{array}\right) \in D^{p \times(p+1)}$ admits a right-inverse over $D$.

Theorem 1 states a necessary and sufficient condition for the linear multidimensional system $R y=0$ defined by a full row rank matrix $R \in D^{p \times p}$ (i.e., $\operatorname{det} R \neq 0$ ) over the commutative polynomial ring $D=\mathbb{R}\left[x_{1}, \ldots, x_{n}\right]$ to be equivalent to the sole equation $(\operatorname{det} R) z=0$, where $z$ is the last component of the column vector $W^{-1} y$. In terms of module theory, Theorem 1 gives a necessary and sufficient condition for the $D$-module $M=D^{1 \times p} /\left(D^{1 \times p} R\right)$ finitely presented by the matrix $R$ to be cyclic, i.e., to be generated by one element (namely, the residue class of the row vector $(0 \ldots 01) \in D^{1 \times p}$ in $\left.M\right)$.

The purpose of this paper is to show that Theorem 1 has deep connections with a result obtained by J.-P. Serre in 39 concerning the number of generators of modules finitely presented by full row rank matrices with entries in the commutative polynomial ring $D=k\left[x_{1}, \ldots, x_{n}\right]$, where $k$ is a field. The main motivation of [39] was the problem called the efficient generation of ideals of $D$ - which aims at finding the minimal number of generators of an ideal $I$ of $D-$ and its applications to algebraic geometry and particularly to the study of the complete intersection of affine algebraic varieties of dimension $n-2$. Moreover, as explained in [10], Serre's reduction problem is also related to the existence of a cyclic vector for linear systems of ordinary differential equations with coefficients in a differential ring or field.

In this paper, we develop a constructive approach to Serre's reduction and we give necessary and sufficient conditions for a full row rank matrix $R \in D^{q \times p}$ (i.e., the rows of $R$ are left $D$ linearly independent) to be equivalent to the diagonal matrix $\operatorname{diag}\left(I_{r}, \bar{R}\right)$ formed by the $r \times r$ identity matrix $I_{r}$ and $\bar{R} \in D^{(q-r) \times(p-r)}$. These conditions generalize Theorem 1 for different classes of linear functional systems (e.g., systems of ordinary or partial differential equations, systems of differential time-delay equations, systems of difference equations) for matrices of 
functional operators which are not necessarily square. Following Serre's approach ([39]), our results use module theory (e.g., Baer extensions ([38, 35])) and homological algebra ([38]).

Finally, based on the resolution of algebraic Riccati equations of the form $\Lambda R \Lambda=\Lambda$, Serre's reduction was also studied in [8, 9] as a particular case of the decomposition problem which aims at studying when there exist two unimodular matrices $V \in \mathrm{GL}_{q}(D)$ and $W \in \mathrm{GL}_{p}(D)$ such that $V R W=\operatorname{diag}\left(\bar{R}_{11}, \bar{R}_{22}\right)\left(\bar{R}_{11}\right.$ being $I_{r}$ in the case of Serre's reduction). However, the constructive methods developed in this paper are dedicated to Serre's reduction, and thus are more efficient than the ones developed in [8] as we shall illustrate it with explicit examples.

\section{A pedestrian approach to Baer extensions}

In what follows, $D$ will denote a noncommutative noetherian domain, namely, a unital ring satisfying that $d d^{\prime}$ is not necessarily equal to $d^{\prime} d$ for $d, d^{\prime} \in D$, containing no nontrivial zerodivisors, i.e., $d d^{\prime}=0$ implies $d=0$ or $d^{\prime}=0$, and every left (resp., right) ideal of $D$ is finitely generated, i.e., can be generated by a finite family of elements of $D$ as a left (resp., right) $D$ module ([24, 38]). Moreover, we shall denote by $D^{1 \times p}$ (resp., $D^{q}$ ) the left (resp., right) $D$-module formed by row (resp., column) vectors of length $p$ (resp., $q$ ) with entries in $D$ and by $R \in D^{q \times p}$ a $q \times p$ matrix $R$ with entries in $D$. Moreover, we shall use the following notations:

$$
\begin{array}{rlrl}
. R: D^{1 \times q} & \longrightarrow D^{1 \times p} & R .: D^{p} & \longrightarrow D^{q} \\
\mu & \longmapsto \mu R, & \eta & \longmapsto R .
\end{array}
$$

In what follows, we shall assume that $p \geq q$ and $R$ has full row rank, namely

$$
\operatorname{ker}_{D}(. R) \triangleq\left\{\mu \in D^{1 \times q} \mid \mu R=0\right\}=0,
$$

i.e., that the rows of $R$ are left $D$-linearly independent, namely, $\mu R=0$ implies $\mu=0$.

Since $\operatorname{im}_{D}(. R) \triangleq\left\{\lambda \in D^{1 \times p} \mid \exists \mu \in D^{1 \times q}: \lambda=\mu R\right\}$, simply denoted by $D^{1 \times q} R$, is a left $D$-submodule of $D^{1 \times p}$, we can introduce the quotient/factor left $D$-module $M=D^{1 \times p} /\left(D^{1 \times q} R\right)$ and the left $D$-homomorphism (i.e., the left $D$-linear application) $\pi: D^{1 \times p} \longrightarrow M$ which sends an element $\lambda \in D^{1 \times p}$ to its residue class $\pi(\lambda)$ in $M$ (i.e., $\pi(\lambda)=\pi\left(\lambda^{\prime}\right)$ iff there exists $\mu \in D^{1 \times q}$ such that $\left.\lambda-\lambda^{\prime}=\mu R\right)$. The left $D$-module $M$ is then said to be finitely presented by $R([38])$. We have the following short exact sequence of left $D$-modules

$$
0 \longrightarrow D^{1 \times q} \stackrel{. R}{\longrightarrow} D^{1 \times p} \stackrel{\pi}{\longrightarrow} M \longrightarrow 0,
$$

namely,.$R$ is an injective left $D$-homomorphism $\left(\right.$ since $\left.\operatorname{ker}_{D}(. R)=0\right), \operatorname{ker}_{D} \pi=D^{1 \times q} R$ and $\pi$ is a surjective left $D$-homomorphism (since, by definition of $M$, every element $m \in M$ has the form $m=\pi(\lambda)$ for a certain $\left.\lambda \in D^{1 \times p}\right)$.

Let us describe the left $D$-module $M=D^{1 \times p} /\left(D^{1 \times q} R\right)$ in terms of generators and relations. Let $\left\{f_{j}\right\}_{j=1, \ldots, p}$ be the standard basis of the left $D$-module $D^{1 \times p}$, namely, $f_{j}$ is the row vector of length $p$ with 1 at the $j^{\text {th }}$ position and 0 elsewhere, and $y_{j} \triangleq \pi\left(f_{j}\right) \in M$ for all $j=1, \ldots, p$. Since every $m \in M$ has the form $m=\pi(\lambda)$ for a certain row vector $\lambda=\left(\lambda_{1} \ldots \lambda_{p}\right) \in D^{1 \times p}$,

$$
m=\pi\left(\sum_{j=1}^{p} \lambda_{j} f_{j}\right)=\sum_{j=1}^{p} \lambda_{j} \pi\left(f_{j}\right)=\sum_{j=1}^{p} \lambda_{j} y_{j},
$$


which shows that every element $m$ of $M$ can be written as a left $D$-linear combination of the $y_{j}$ 's, i.e., $\left\{y_{j}\right\}_{j=1, \ldots, p}$ is a family of generators of $M$. Moreover, if $R_{i}$ denotes the $i^{\text {th }}$ row of the matrix $R \in D^{q \times p}$, then $R_{i} \in D^{1 \times q} R$ which yields $\pi\left(R_{i \bullet}\right)=0$ for all $i=1, \ldots, q$, i.e.,

$$
\forall i=1, \ldots, q, \quad \pi\left(\sum_{j=1}^{p} R_{i j} f_{j}\right)=\sum_{j=1}^{p} R_{i j} \pi\left(f_{j}\right)=\sum_{j=1}^{p} R_{i j} y_{j}=0,
$$

and shows that the generators $y_{j}$ 's of $M$ satisfy the left $D$-linear relations (2) and all their left $D$-linear combinations.

Let us now consider the following two matrices

$$
\Lambda \in D^{q \times(q-r)}, \quad 0 \leq r \leq q-1, \quad P \triangleq(R \quad-\Lambda) \in D^{q \times(p+q-r)},
$$

the left $D$-module $E=D^{1 \times(p+q-r)} /\left(D^{1 \times q} P\right)$ finitely presented by the full row rank matrix $P$ and the following short exact sequence of left $D$-modules

$$
0 \longrightarrow D^{1 \times q} \stackrel{. P}{\longrightarrow} D^{1 \times(p+q-r)} \stackrel{\varrho}{\longrightarrow} E \longrightarrow 0,
$$

where $\varrho: D^{1 \times(p+q-r)} \longrightarrow E$ denotes the canonical projection onto $E$, i.e., the left $D$-homomorphism which sends $\zeta \in D^{1 \times(p+q-r)}$ to its residue class $\varrho(\zeta)$ in $E$.

Let us study the connections between the left $D$-modules $M$ and $E$. If we introduce the matrix $X=\left(\begin{array}{ll}I_{p}^{T} & 0^{T}\end{array}\right)^{T} \in D^{(p+q-r) \times p}$, then the identity $R=P X$ induces the following commutative exact diagram of left $D$-modules

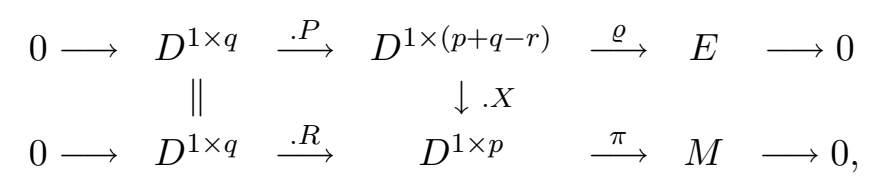

as well as the well-defined left $D$-homomorphism $\beta: E \longrightarrow M$ defined by:

$$
\forall \mu_{1} \in D^{1 \times p}, \quad \forall \mu_{2} \in D^{1 \times(q-r)}, \quad \beta\left(\varrho\left(\left(\begin{array}{ll}
\mu_{1} & \mu_{2}
\end{array}\right)\right)\right)=\pi\left(\left(\mu_{1} \quad \mu_{2}\right) X\right)=\pi\left(\mu_{1}\right) .
$$

For every $m \in M$, there exists $\mu_{1} \in D^{1 \times p}$ such that $m=\pi\left(\mu_{1}\right)$ and thus $m=\beta\left(\varrho\left(\left(\mu_{1} \quad 0\right)\right)\right)$, which proves that $\beta$ is surjective, i.e., $\operatorname{im} \beta=M$.

Let us study $\operatorname{ker} \beta$. An element $\varrho\left(\left(\begin{array}{ll}\mu_{1} & \mu_{2}\end{array}\right)\right) \in \operatorname{ker} \beta$ satisfies $\pi\left(\mu_{1}\right)=0$, i.e., $\mu_{1}=\nu R$ for a certain $\nu \in D^{1 \times q}$. Since $\varrho\left(\left(\begin{array}{ll}\nu R & -\nu \Lambda\end{array}\right)\right)=0$ yields $\varrho\left(\left(\begin{array}{ll}\nu R & 0\end{array}\right)\right)=\varrho\left(\left(\begin{array}{ll}0 & \nu \Lambda\end{array}\right)\right)$, we obtain:

$$
\begin{aligned}
\operatorname{ker} \beta & =\left\{\varrho\left(\left(\begin{array}{ll}
\nu R & \mu_{2}
\end{array}\right)\right)=\varrho\left(\left(\begin{array}{ll}
0 & \mu_{2}+\nu \Lambda
\end{array}\right) \mid \nu \in D^{1 \times q}, \mu_{2} \in D^{1 \times(q-r)}\right\}\right. \\
& =\left\{\varrho\left(\left(\begin{array}{ll}
0 & \xi
\end{array}\right)\right) \mid \xi \in D^{1 \times(q-r)}\right\} .
\end{aligned}
$$

Let $\gamma: D^{1 \times(q-r)} \longrightarrow \operatorname{ker} \beta$ be the left $D$-isomorphism defined by $\gamma(\xi)=\varrho\left(\left(\begin{array}{ll}0 & \xi\end{array}\right)\right)$ for all $\xi \in D^{1 \times(q-r)}$ (i.e., $\alpha$ is injective and surjective). The canonical short exact sequence

$$
0 \longrightarrow \operatorname{ker} \beta \stackrel{i}{\longrightarrow} E \stackrel{\beta}{\longrightarrow} \operatorname{im} \beta \longrightarrow 0
$$

then yields the following one

$$
0 \longrightarrow D^{1 \times(q-r)} \stackrel{\alpha}{\longrightarrow} E \stackrel{\beta}{\longrightarrow} M \longrightarrow 0
$$


where $\alpha=i \circ \gamma$. The short exact sequence (4) is called a Baer extension of $D^{1 \times(q-r)}$ by $M($ (38) and we shall simply say an extension of $D^{1 \times(q-r)}$ by $M$.

Let us now introduce the following matrices

$$
\Theta \in D^{p \times(q-r)}, \quad \bar{\Lambda} \triangleq \Lambda+R \Theta \in D^{q \times(q-r)}, \quad \bar{P} \triangleq(R \quad-\bar{\Lambda}) \in D^{q \times(p+q-r)},
$$

and the left $D$-module $\bar{E}=D^{1 \times(p+q-r)} /\left(D^{1 \times q} \bar{P}\right)$ finitely presented by $\bar{P}$. Let us also denote by $\bar{\varrho}: D^{1 \times(p+q-r)} \longrightarrow \bar{E}$ the canonical projection onto $\bar{E}$. Doing as previously with the left $D$-module $\bar{E}$, we obtain the extension of $D^{1 \times(q-r)}$ by $M$ defined by

$$
0 \longrightarrow D^{1 \times(q-r)} \stackrel{\bar{\alpha}}{\longrightarrow} \bar{E} \stackrel{\bar{\beta}}{\longrightarrow} M \longrightarrow 0
$$

with the following notations:

$$
\begin{aligned}
\forall \xi \in D^{1 \times(q-r)}, \quad \bar{\alpha}(\xi) & =\bar{\varrho}\left(\left(\begin{array}{ll}
0 & \xi
\end{array}\right)\right), \\
\forall \mu_{1} \in D^{1 \times p}, \forall \mu_{2} \in D^{1 \times(q-r)}, \quad \bar{\beta}\left(\bar{\varrho}\left(\left(\begin{array}{ll}
\mu_{1} & \mu_{2}
\end{array}\right)\right)\right) & =\pi\left(\mu_{1}\right) .
\end{aligned}
$$

If we introduce the general linear group $\mathrm{GL}_{r}(D)$ of $D$ of index $r$ defined by

$$
\mathrm{GL}_{r}(D)=\left\{U \in D^{r \times r} \mid \exists V \in D^{r \times r}: U V=V U=I_{r}\right\}
$$

and the unimodular matrix $V$, namely, $V \in \mathrm{GL}_{p+q-r}(D)$, defined by

$$
V=\left(\begin{array}{cc}
I_{p} & \Theta \\
0 & I_{q-r}
\end{array}\right),
$$

then the identity $P=\bar{P} V$ induces the following commutative exact diagram:

$$
\begin{array}{ccccccc}
0 \longrightarrow & D^{1 \times q} & \stackrel{.}{\longrightarrow} & D^{1 \times(p+q-r)} & \stackrel{\bar{\varrho}}{\longrightarrow} & \bar{E} & \longrightarrow 0 \\
\| & & \downarrow . V & & & \\
0 \longrightarrow & D^{1 \times q} & \stackrel{. P}{\longrightarrow} & D^{1 \times(p+q-r)} & \stackrel{\varrho}{\longrightarrow} & E & \longrightarrow 0 .
\end{array}
$$

Since $V \in \operatorname{GL}_{p+q-r}(D)$, we get the left $D$-isomorphism $\psi: \bar{E} \longrightarrow E$ defined by

$$
\psi\left(\bar{\varrho}\left(\left(\begin{array}{ll}
\mu_{1} & \mu_{2}
\end{array}\right)\right)\right)=\varrho\left(\left(\begin{array}{ll}
\mu_{1} & \mu_{2}
\end{array}\right) V\right)=\varrho\left(\left(\begin{array}{ll}
\mu_{1} & \left.\left.\mu_{1} \Theta+\mu_{2}\right)\right),
\end{array}\right.\right.
$$

for all $\mu_{1} \in D^{1 \times p}$ and all $\mu_{2} \in D^{1 \times(q-r)}$. Then, for every $\xi \in D^{1 \times(q-r)}$, we have

$$
(\psi \circ \bar{\alpha})(\xi)=\psi\left(\bar{\varrho}\left(\left(\begin{array}{ll}
0 & \xi
\end{array}\right)\right)\right)=\varrho\left(\left(\begin{array}{ll}
0 & \xi
\end{array}\right)\right)=\alpha(\xi),
$$

which proves $\alpha=\psi \circ \bar{\alpha}$. Now, for every $\mu_{1} \in D^{1 \times p}$ and every $\mu_{2} \in D^{1 \times(q-r)}$,

$$
(\beta \circ \psi)\left(\bar{\varrho}\left(\left(\mu_{1} \quad \mu_{2}\right)\right)\right)=\beta\left(\varrho\left(\left(\mu_{1} \quad \mu_{2}+\mu_{1} \Theta\right)\right)\right)=\pi_{1}\left(\mu_{1}\right)=\bar{\beta}\left(\varrho\left(\left(\mu_{1} \quad \mu_{2}\right)\right)\right),
$$

which proves $\bar{\beta}=\beta \circ \psi$. Therefore, we obtain the commutative exact diagram:

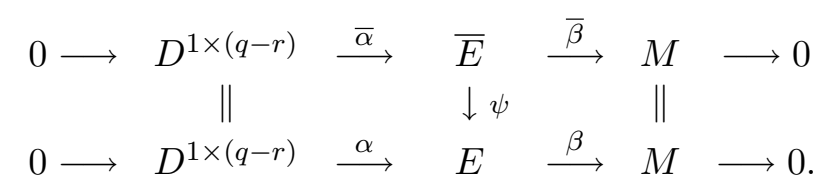

We are then led to the following definition of equivalent extensions.

$\mathrm{RR} \mathrm{n}^{\circ} 7214$ 
Definition 1 ([38]). Two extensions of $D^{1 \times(q-r)}$ by $M$

$$
e: 0 \longrightarrow D^{1 \times(q-r)} \stackrel{\alpha}{\longrightarrow} E \stackrel{\beta}{\longrightarrow} M \longrightarrow 0, \quad \bar{e}: 0 \longrightarrow D^{1 \times(q-r)} \stackrel{\bar{\alpha}}{\longrightarrow} \bar{E} \stackrel{\bar{\beta}}{\longrightarrow} M \longrightarrow 0,
$$

are said to be equivalent if there exists a left $D$-homomorphism $\psi: \bar{E} \longrightarrow E$ satisfying $\alpha=\psi \circ \bar{\alpha}$ and $\bar{\beta}=\beta \circ \psi$, i.e., if $(5)$ is a commutative exact diagram.

If $e$ and $\bar{e}$ are equivalent extensions, then we can easily check that $\psi$ is necessarily a left $D$-isomorphism (e.g., apply the snake lemma ([38]) to (5)). Hence, we can easily check that the equivalence defined in Definition 1 is an equivalence relation $\sim$ on the set of extensions of $D^{1 \times(q-r)}$ by $M([38])$. We denote by $\mathrm{e}_{D}\left(M, D^{1 \times(q-r)}\right)$ the set of all equivalence classes of extensions of $D^{1 \times(q-r)}$ by $M$ and $[e]$ the equivalence class of the extension $e$ in $\mathrm{e}_{D}\left(M, D^{1 \times(q-r)}\right)$.

The previous results show that the extensions of $D^{1 \times(q-r)}$ by $M$ defined by $E$ and $\bar{E}$, i.e., by means of the matrices $\Lambda$ and $\bar{\Lambda}=\Lambda+R \Theta$ for $\Theta \in D^{p \times(q-r)}$, are equivalent, and thus they define the same equivalence class in $\mathrm{e}_{D}\left(M, D^{1 \times(q-r)}\right)$.

Let us now explain another relation between $\mathrm{e}_{D}\left(M, D^{1 \times(q-r)}\right)$ and the matrices $\Lambda$ and $\bar{\Lambda}=\Lambda+R \Theta$. To do that, we first need to introduce the right $D$-module

$$
\operatorname{ext}_{D}^{1}\left(M, D^{1 \times(q-r)}\right) \triangleq D^{q \times(q-r)} /\left(R D^{p \times(q-r)}\right)
$$

called the first extension right $D$-module of $M$ with value in $D^{1 \times(q-r)}$. We use the notation $\operatorname{ext}_{D}^{1}\left(M, D^{1 \times(q-r)}\right)$ since we can prove that $D^{q \times(q-r)} /\left(R D^{p \times(q-r)}\right)$ depends only on $M$ and $D^{1 \times(q-r)}$ and not on the choice of the matrix $R$ which presents the left $D$-module $M$ (see, e.g., [38]). Moreover, since $R$ has full row rank, the higher extension right $D$-modules $\operatorname{ext}_{D}^{i}\left(M, D^{1 \times(q-r)}\right)$ of $M$ with values in $D^{1 \times(q-r)}$ are reduced to 0 for all $i \geq 2$ (see, e.g., [38]).

Remark 1. We recall that the vanishing of the left $D$-modules $\operatorname{ext}_{D}^{i}(N, D)$ for $i \in \mathbb{N}=\{0,1, \ldots\}$, where the right $D$-module $N=D^{q} /\left(R D^{p}\right)$ is called the Auslander transpose of left $D$-module $M=D^{1 \times p} /\left(D^{1 \times q} R\right)$, characterizes the module properties of $M([6,28])$. Moreover, if $\mathcal{F}$ is a left $D$-module, $\operatorname{hom}_{D}(M, \mathcal{F})$ the abelian group of left $D$-homomorphisms from $M$ to $\mathcal{F}$ and $\cong$ the relation of being isomorphic, then $\operatorname{ker}_{\mathcal{F}}(R.) \triangleq\left\{\eta \in \mathcal{F}^{p} \mid R \eta=0\right\} \cong \operatorname{hom}_{D}(M, \mathcal{F})$, which proves that the $\mathcal{F}$-solution space of the linear system $R \eta=0$ is intrinsically defined by $\operatorname{hom}_{D}(M, \mathcal{F})$. This result, classical in homological algebra, was first pointed out by Malgrange ([22]). In particular, the left $D$-isomorphism $\chi: \operatorname{ker}_{\mathcal{F}}(R.) \longrightarrow \operatorname{hom}_{D}(M, \mathcal{F})$ is defined by $\chi(\eta)(\pi(\lambda))=\lambda \eta$ for all $\eta \in \operatorname{ker}_{\mathcal{F}}\left(R\right.$.) and all $\lambda \in D^{1 \times p}$ and its inverse $\chi^{-1}: \operatorname{hom}_{D}(M, \mathcal{F}) \longrightarrow \operatorname{ker}_{\mathcal{F}}(R$. $)$ is $\chi^{-1}(\phi)=\left(\phi\left(y_{1}\right) \ldots \phi\left(y_{p}\right)\right)^{T}$ for all $\phi \in \operatorname{hom}_{D}(M, \mathcal{F})$. Hence, the vanishing of the $\operatorname{ext}_{D}^{i}(N, D)$ 's for $i \in \mathbb{N}$ characterizes structural properties of $\operatorname{ker} \mathcal{F}(R).([6,28])$.

If $\rho: D^{q \times(q-r)} \longrightarrow \operatorname{ext}_{D}^{1}\left(M, D^{1 \times(q-r)}\right)=D^{q \times(q-r)} /\left(R D^{p \times(q-r)}\right)$ is the canonical projection, then we have

$$
\forall \Theta \in D^{p \times(q-r)}, \quad \rho(\bar{\Lambda})=\rho(\Lambda+R \Theta)=\rho(\Lambda),
$$

i.e., $\Lambda$ and $\bar{\Lambda}=\Lambda+R \Theta$ belong to the same residue class in $\operatorname{ext}_{D}^{1}\left(M, D^{1 \times(q-r)}\right)$.

We have just proved that every element $\rho(\Lambda) \in \operatorname{ext}_{D}^{1}\left(M, D^{1 \times(q-r)}\right)$ defines the unique equivalence class $[e]$ of extensions of $D^{1 \times(q-r)}$ by $M$, where

$$
e: 0 \longrightarrow D^{1 \times(q-r)} \stackrel{\alpha}{\longrightarrow} E \stackrel{\beta}{\longrightarrow} M \longrightarrow 0,
$$


and the left $D$-module $E$ is finitely presented by the matrix $P=(R-\Lambda)$, i.e.:

$$
E=D^{1 \times(p+q-r)} /\left(D^{1 \times q} P\right) .
$$

Let us now study the converse of the last result. We first consider the following extension of $D^{1 \times(q-r)}$ by $M$ :

$$
0 \longrightarrow D^{1 \times(q-r)} \stackrel{\varepsilon}{\longrightarrow} F \stackrel{\delta}{\longrightarrow} M \longrightarrow 0 .
$$

Let $\left\{f_{i}\right\}_{i=1, \ldots, p}$ be the standard basis of $D^{1 \times p}$, namely, $f_{i}$ is the row vector with 1 at the $i^{\text {th }}$ position and 0 elsewhere. Since the left $D$-homomorphism $\delta$ is surjective, there exists $\zeta_{i} \in F$ such that $\delta\left(\zeta_{i}\right)=\pi\left(f_{i}\right) \in M$ for all $i=1, \ldots, p$. Then, we get

$$
\delta\left(\sum_{k=1}^{p} R_{j k} \zeta_{k}\right)=\sum_{k=1}^{p} R_{j k} \delta\left(\zeta_{k}\right)=\sum_{k=1}^{p} R_{j k} \pi\left(f_{k}\right)=\pi\left(\sum_{k=1}^{p} R_{j k} f_{k}\right)=\pi\left(R_{j \bullet}\right)=0,
$$

for all $j=1, \ldots, q$, where $R_{j}$ denotes the $j^{\text {th }}$ row of the matrix $R$. Since $\operatorname{ker} \delta=\operatorname{im} \varepsilon$ and $\varepsilon$ is injective, there exists a unique element $\lambda_{j} \in D^{1 \times(q-r)}$ such that $\sum_{k=1}^{p} R_{j k} \zeta_{k}=\varepsilon\left(\lambda_{j}\right)$. If we denote by $\Lambda=\left(\lambda_{1}^{T} \ldots \lambda_{q}^{T}\right)^{T} \in D^{q \times(q-r)}$, then we get $\rho(\Lambda) \in \operatorname{ext}_{D}^{1}\left(M, D^{1 \times(q-r)}\right)$. Let us check that the residue class $\rho(\Lambda)$ of $\Lambda$ in $\operatorname{ext}_{D}^{1}\left(M, D^{1 \times(q-r)}\right)$ is well-defined, i.e., it does not depend on the choice of the pre-images $\zeta_{i}$ 's of the $\pi\left(f_{i}\right)^{\prime}$ 's. Let us consider other pre-images $\bar{\zeta}_{i}$ 's of the $\pi\left(f_{i}\right)$, i.e., $\delta\left(\bar{\zeta}_{i}\right)=\pi\left(f_{i}\right)$ for all $i=1, \ldots, p$. Using the same arguments, there exists $\bar{\lambda}_{j} \in D^{1 \times(q-r)}$ such that $\sum_{k=1}^{p} R_{j k} \bar{\zeta}_{k}=\varepsilon\left(\bar{\lambda}_{j}\right)$ for all $j=1, \ldots, q$. But, $\delta\left(\bar{\zeta}_{i}\right)=\delta\left(\zeta_{i}\right)$ yields $\delta\left(\bar{\zeta}_{i}-\zeta_{i}\right)=0$, i.e., $\bar{\zeta}_{i}-\zeta_{i} \in \operatorname{ker} \delta=\operatorname{im} \varepsilon$ and thus there exists $\theta_{i} \in D^{1 \times(q-r)}$ such that $\bar{\zeta}_{i}=\zeta_{i}+\varepsilon\left(\theta_{i}\right)$ and thus:

$$
\varepsilon\left(\bar{\lambda}_{j}\right)=\sum_{k=1}^{p} R_{j k} \bar{\zeta}_{k}=\varepsilon\left(\lambda_{j}\right)+\sum_{k=1}^{p} R_{j k} \varepsilon\left(\theta_{k}\right)=\varepsilon\left(\lambda_{j}+\sum_{k=1}^{p} R_{j k} \theta_{k}\right) .
$$

If we introduce the following two matrices

$$
\bar{\Lambda}=\left(\begin{array}{c}
\bar{\lambda}_{1} \\
\vdots \\
\bar{\lambda}_{q}
\end{array}\right) \in D^{q \times(q-r)}, \quad \Theta=\left(\begin{array}{c}
\theta_{1} \\
\vdots \\
\theta_{p}
\end{array}\right) \in D^{p \times(q-r)},
$$

then, since $\varepsilon$ is injective, (8) yields $\bar{\lambda}_{j}=\lambda_{j}+\sum_{k=1}^{p} R_{j k} \theta_{k}$ for all $j=1, \ldots, q$, i.e., $\bar{\Lambda}=\Lambda+R \Theta$, and thus $\rho(\bar{\Lambda})=\rho(\Lambda+R \widehat{\Theta})=\rho(\Lambda)$, which finally proves that every extension $(7)$ of $D^{1 \times(q-r)}$ by $M$ defines a unique element $\rho(\Lambda)$ of the right $D$-module $\operatorname{ext}_{D}^{1}\left(M, D^{1 \times(q-r)}\right)$. Finally, let us prove that every extension in the same equivalence class of 7 in $\mathrm{e}_{D}\left(M, D^{1 \times(q-r)}\right)$ defines the same element $\rho(\Lambda) \in \operatorname{ext}_{D}^{1}\left(M, D^{1 \times(q-r)}\right)$. Let us consider an extension of $D^{1 \times(q-r)}$ by $M$ in the same equivalence class of (7). Then, the following commutative exact diagram

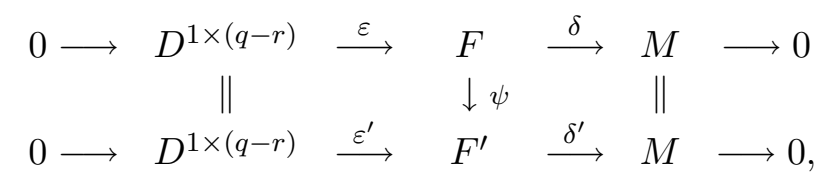

holds for a certain left $D$-isomorphism $\psi$. Using $\delta^{\prime} \circ \psi=\delta$, we obtain that $\delta^{\prime}\left(\psi\left(\zeta_{i}\right)\right)=\pi\left(f_{i}\right)$ for all $i=1, \ldots, p$, and applying $\psi$ to $\sum_{k=1}^{p} R_{j k} \zeta_{k}=\varepsilon\left(\lambda_{j}\right)$ and using $\varepsilon^{\prime}=\psi \circ \varepsilon$, we get $\sum_{k=1}^{p} R_{j k} \psi\left(\zeta_{k}\right)=\varepsilon^{\prime}\left(\lambda_{j}\right)$ for all $j=1, \ldots, q$, which gives the same matrix $\Lambda=\left(\lambda_{1}^{T} \ldots \lambda_{q}^{T}\right)$ as previously and thus the same residue class $\rho(\Lambda)$ in $\operatorname{ext}_{D}^{1}\left(M, D^{1 \times(q-r)}\right)$. 
Hence, there is a one-to-one correspondence between the elements of the right $D$-module $\operatorname{ext}_{D}^{1}\left(M, D^{1 \times(q-r)}\right)$ and the equivalence classes of extensions of $D^{1 \times(q-r)}$ by $M$. This result is attributed to Baer ([1]). An important consequence of this result is that every equivalence class of extensions of $D^{1 \times(q-r)}$ by $M$ contains a representative defined by the short exact sequence

$$
0 \longrightarrow D^{1 \times(q-r)} \stackrel{\alpha}{\longrightarrow} E_{\rho(\Lambda)} \stackrel{\beta}{\longrightarrow} M \longrightarrow 0
$$

where $E_{\rho(\Lambda)}=D^{1 \times(p+q-r)} /\left(D^{1 \times q}(R-\Lambda)\right)$ for a certain $\Lambda \in D^{q \times(q-r)}$. The Baer sum $\left[e_{1}\right]+\left[e_{2}\right]$ of two equivalence classes $\left[e_{1}\right]$ and $\left[e_{2}\right]$ of extensions of $D^{1 \times(q-r)}$ by $M$, respectively defined by representatives formed by $E_{\rho\left(\Lambda_{1}\right)}$ and $E_{\rho\left(\Lambda_{2}\right)}$, is the equivalence class of the extension defined by $E_{\rho\left(\Lambda_{1}+\Lambda_{2}\right)}$. See [34, 38] for proofs. Endowed with the Baer sum and the neutral element defined by the equivalence class of the extension of $D^{1 \times(q-r)}$ by $M$ defined by the central left $D$-module

$$
E_{\rho(0)}=D^{1 \times(p+q-r)} /\left(D^{1 \times q}(R \quad 0)\right)=D^{1 \times(q-r)} \oplus M,
$$

i.e., the equivalence class of the following split short exact sequence

$$
0 \longrightarrow D^{1 \times(q-r)} \stackrel{\alpha}{\longrightarrow} D^{1 \times(q-r)} \oplus M \stackrel{\beta}{\longrightarrow} M \longrightarrow 0,
$$

we can prove that $\mathrm{e}_{D}\left(M, D^{1 \times(q-r)}\right)$ becomes an abelian group which is isomorphic to the abelian group $\operatorname{ext}_{D}^{1}\left(M, D^{1 \times(q-r)}\right)$ (see, e.g., [38]).

We obtain the following important result in homological algebra.

Theorem $2([38]) \cdot \operatorname{ext}_{D}^{1}\left(M, D^{1 \times(q-r)}\right) \cong \mathrm{e}_{D}\left(M, D^{1 \times(q-r)}\right)$.

A classical result in homological algebra asserts that

$$
\operatorname{ext}_{D}^{1}\left(M, D^{1 \times(q-r)}\right) \cong \operatorname{ext}_{D}^{1}(M, D) \otimes_{D} D^{1 \times(q-r)} \cong \operatorname{ext}_{D}^{1}(M, D)^{1 \times(q-r)},
$$

for all left $D$-modules $M$, where $\otimes_{D}$ denotes the tensor product (see, e.g., [38]). Substituting $r=q-1$ in $(6)$, we get $\operatorname{ext}_{D}^{1}(M, D)=D^{q} /\left(R D^{p}\right)$ (i.e., $\operatorname{ext}_{D}^{1}(M, D)$ is the Auslander transpose $N$ of $M$ by Remark 1). If $\tau: D^{q} \longrightarrow \operatorname{ext}_{D}^{1}(M, D)$ is the canonical projection onto $\operatorname{ext}_{D}^{1}(M, D)$, then $\rho=\tau \otimes \operatorname{id}_{(q-r)}$, i.e., an element $\rho(\Lambda)$ can be interpreted as a row vector of length $q-r$ formed by the elements $\tau\left(\Lambda_{\bullet}\right) \in \operatorname{ext}_{D}^{1}(M, D)$, where $\Lambda_{\bullet} i$ denotes the $i^{\text {th }}$ column of the matrix $\Lambda \in D^{q \times(q-r)}$, i.e., $\rho(\Lambda)=\left(\tau\left(\Lambda_{\bullet}\right) \ldots \tau\left(\Lambda_{\bullet}(q-r)\right)\right) \in \operatorname{ext}_{D}^{1}(M, D)^{1 \times(q-r)}$.

For more details on the applications of Baer extensions to mathematical systems theory, see [31, 32, 34, 35].

To finish with this section, we give more examples of extensions which will be used later on. To do that, we first recall two useful lemmas. The first one gives a finite presentation of a left $D$-module of the form $\left(D^{1 \times q^{\prime}} R^{\prime}\right) /\left(D^{1 \times q} R\right)$, whenever $D^{1 \times q} R \subseteq D^{1 \times q^{\prime}} R^{\prime} \subseteq D^{1 \times p}$. This result was first obtained in [8]. For the sake of completeness, we recall its proof.

Lemma 1 (Lemma 3.1 of [8]). Let $R \in D^{q \times p}$ and $R^{\prime} \in D^{q^{\prime} \times p}$ be two matrices which satisfy $D^{1 \times q} R \subseteq D^{1 \times q^{\prime}} R^{\prime}$ and $R^{\prime \prime} \in D^{q \times q^{\prime}}$ and $T^{\prime} \in D^{r^{\prime} \times q^{\prime}}$ such that $R=R^{\prime \prime} R^{\prime}$ and $\operatorname{ker}_{D}\left(. R^{\prime}\right)=$ $D^{1 \times r^{\prime}} T^{\prime}$. Let us also consider the following canonical projections:

$$
\begin{aligned}
& \pi_{1}: D^{1 \times q^{\prime}} R^{\prime} \longrightarrow M_{1}=\left(D^{1 \times q^{\prime}} R^{\prime}\right) /\left(D^{1 \times q} R\right), \\
& \pi_{2}: D^{1 \times q^{\prime}} \longrightarrow M_{2}=D^{1 \times q^{\prime}} /\left(D^{1 \times q} R^{\prime \prime}+D^{1 \times r^{\prime}} T^{\prime}\right) .
\end{aligned}
$$


Then, the left D-homomorphism $\psi$ defined by

$$
\begin{aligned}
\psi: M_{2} & \longrightarrow M_{1} \\
m_{2}=\pi_{2}(\lambda) & \longmapsto \psi\left(m_{2}\right)=\pi_{1}\left(\lambda R^{\prime}\right),
\end{aligned}
$$

is an isomorphism and its inverse $\phi=\psi^{-1}$ is defined by:

$$
\begin{aligned}
\phi: M_{1} & \longrightarrow M_{2} \\
m_{1}=\pi_{1}\left(\lambda R^{\prime}\right) & \longmapsto \phi\left(m_{1}\right)=\pi_{2}(\lambda) .
\end{aligned}
$$

In other words, we have the following isomorphism of left D-modules:

$$
\left(D^{1 \times q^{\prime}} R^{\prime}\right) /\left(D^{1 \times q} R\right) \cong D^{1 \times q^{\prime}} /\left(D^{1 \times q} R^{\prime \prime}+D^{1 \times r^{\prime}} T^{\prime}\right) .
$$

Proof. Let us first prove that $\psi$ is a well-defined left $D$-homomorphism. Let us assume that $m_{2}=\pi_{2}(\lambda)=\pi_{2}\left(\lambda^{\prime}\right)$, where $\lambda, \lambda^{\prime} \in D^{1 \times q^{\prime}}$. Then, $\pi_{2}\left(\lambda-\lambda^{\prime}\right)=0$, i.e., $\lambda-\lambda^{\prime} \in D^{1 \times q} R^{\prime \prime}+D^{1 \times r^{\prime}} T^{\prime}$ so that there exist $\mu \in D^{1 \times q}$ and $\nu \in D^{1 \times r^{\prime}}$ such that $\lambda-\lambda^{\prime}=\mu R^{\prime \prime}+\nu T^{\prime}$. We then have:

$$
\begin{gathered}
\left(\lambda-\lambda^{\prime}\right) R^{\prime}=\left(\mu R^{\prime \prime}+\nu T^{\prime}\right) R^{\prime}=\mu R \Rightarrow \pi_{1}\left(\left(\lambda-\lambda^{\prime}\right) R^{\prime}\right)=\pi_{1}(\mu R)=0 \\
\Rightarrow \pi_{1}\left(\lambda^{\prime} R^{\prime}\right)=\pi_{1}\left(\lambda R^{\prime}\right)=\psi\left(m_{2}\right) .
\end{gathered}
$$

Let us now prove that $\phi$ is also well-defined. Let us suppose that $m_{1}=\pi_{1}\left(\lambda R^{\prime}\right)=\pi_{1}\left(\lambda^{\prime} R^{\prime}\right)$, where $\lambda, \lambda^{\prime} \in D^{1 \times q^{\prime}}$. Then, $\pi_{1}\left(\lambda R^{\prime}\right)-\pi_{1}\left(\lambda^{\prime} R^{\prime}\right)=\pi_{1}\left(\left(\lambda-\lambda^{\prime}\right) R^{\prime}\right)=0$, i.e., $\left(\lambda-\lambda^{\prime}\right) R^{\prime} \in D^{1 \times q} R$, and thus, there exists $\mu \in D^{1 \times q}$ such that $\left(\lambda-\lambda^{\prime}\right) R^{\prime}=\mu R$. Now, using $R=R^{\prime \prime} R^{\prime}$, we get $\left(\lambda-\lambda^{\prime}-\mu R^{\prime \prime}\right) R^{\prime}=0$ so that $\lambda-\lambda^{\prime}-\mu R^{\prime \prime} \in \operatorname{ker}_{D}\left(. R^{\prime}\right)=D^{1 \times r^{\prime}} T^{\prime}$. Therefore, there exists $\nu \in D^{1 \times r^{\prime}}$ such that $\lambda-\lambda^{\prime}=\mu R^{\prime \prime}+\nu T^{\prime}$ and thus:

$$
\pi_{2}(\lambda)-\pi_{2}\left(\lambda^{\prime}\right)=\pi_{2}\left(\lambda-\lambda^{\prime}\right)=\pi_{2}\left(\mu R^{\prime \prime}+\nu T^{\prime}\right)=0 .
$$

For every $m_{1}=\pi_{1}\left(\lambda R^{\prime}\right) \in M_{1}$ and every $m_{2}=\pi_{2}(\lambda) \in M_{2}$, where $\lambda \in D^{1 \times q^{\prime}}$, we have

$$
\left\{\begin{array}{l}
(\psi \circ \phi)\left(m_{1}\right)=\psi\left(\pi_{2}(\lambda)\right)=\pi_{1}\left(\lambda R^{\prime}\right)=m_{1}, \\
(\phi \circ \psi)\left(m_{2}\right)=\phi\left(\pi_{1}\left(\lambda R^{\prime}\right)\right)=\pi_{2}(\lambda)=m_{2},
\end{array}\right.
$$

which finally proves that $\psi \circ \phi=\operatorname{id}_{M_{1}}, \phi \circ \psi=\operatorname{id}_{M_{2}}, \psi=\phi^{-1}$ and $(9$ ).

The second one is the classical third isomorphism theorem of module theory.

Lemma 2 ([38]). If $L^{\prime \prime} \subseteq L^{\prime} \subseteq L$ are three left (resp., right) D-modules, then we have the following isomorphism of left (resp., right) D-modules:

$$
L / L^{\prime} \cong\left[L / L^{\prime \prime}\right] /\left[L^{\prime} / L^{\prime \prime}\right] .
$$

Example 1. Let us split the matrix $R \in D^{q \times p}$ as follows:

$$
R=\left(\begin{array}{c}
R_{1} \\
R_{2}
\end{array}\right), \quad R_{1} \in D^{r \times p}, \quad R_{2} \in D^{(q-r) \times p} .
$$

Using the inclusion $D^{1 \times r} R_{1} \subseteq D^{1 \times q} R=D^{1 \times r} R_{1}+D^{1 \times(q-r)} R_{2} \subseteq D^{1 \times p}$ of left $D$-modules, Lemma 2 applied to $L=D^{1 \times p}, L^{\prime}=D^{1 \times q} R$ and $L^{\prime \prime}=D^{1 \times r} R_{1}$ yields the following short exact sequence of left $D$-modules

$$
0 \longrightarrow\left(D^{1 \times q} R\right) /\left(D^{1 \times r} R_{1}\right) \stackrel{\alpha}{\longrightarrow} M_{1}=D^{1 \times p} /\left(D^{1 \times r} R_{1}\right) \stackrel{\beta}{\longrightarrow} M \longrightarrow 0,
$$


where $M_{1}$ is the left $D$-module finitely presented by $R_{1}$ and the left $D$-homomorphisms $\alpha$ and $\beta$ are defined by

$$
\begin{aligned}
& \alpha=\mathrm{id}:\left(D^{1 \times q} R\right) /\left(D^{1 \times r} R_{1}\right) \longrightarrow M_{1} \quad \beta: M_{1} \longrightarrow M \\
& \pi_{1}(\mu R) \longmapsto \pi_{1}(\mu R), \quad \pi_{1}(\lambda) \longmapsto \pi(\lambda),
\end{aligned}
$$

where $\pi_{1}: D^{1 \times p} \longrightarrow M_{1}=D^{1 \times p} /\left(D^{1 \times r} R_{1}\right)$ is the canonical projection onto $M_{1}$. Let $S=$ $\left(\begin{array}{ll}S_{1} & S_{2}\end{array}\right) \in D^{s \times q}$, where $S_{1} \in D^{s \times r}$ and $S_{2} \in D^{s \times(q-r)}$, be such that $\operatorname{ker}_{D}(. R)=D^{1 \times s} S$ and let us introduce the matrix $T=\left(\begin{array}{ll}L^{T} & S^{T}\end{array}\right)^{T} \in D^{(r+s) \times q}$, where $L=\left(\begin{array}{ll}I_{r} & 0\end{array}\right) \in D^{r \times q}$, and $\vartheta: D^{1 \times q} \longrightarrow D^{1 \times q} /\left(D^{1 \times(r+s)} T\right)$ the canonical projection. Then, Lemma 1 yields:

$$
\begin{aligned}
\left(D^{1 \times q} R\right) /\left(D^{1 \times r} R_{1}\right) & \cong D^{1 \times q} /\left(D^{1 \times(r+s)}\left(\begin{array}{cc}
I_{r} & 0 \\
S_{1} & S_{2}
\end{array}\right)\right) \\
& \cong D^{1 \times q} /\left(D^{1 \times(r+s)}\left(\begin{array}{cc}
I_{r} & 0 \\
0 & S_{2}
\end{array}\right)\right) \\
& \cong D^{1 \times(q-r)} /\left(D^{1 \times s} S_{2}\right) .
\end{aligned}
$$

If $\psi: Q \triangleq D^{1 \times(q-r)} /\left(D^{1 \times r} S_{2}\right) \longrightarrow\left(D^{1 \times q} R\right) /\left(D^{1 \times r} R_{1}\right)$ denotes the previous left $D$-isomorphism, then we obtain the following extension of $Q$ by $M$ :

$$
0 \longrightarrow Q \stackrel{\alpha \circ \psi}{\longrightarrow} M_{1} \stackrel{\beta}{\longrightarrow} M \longrightarrow 0 .
$$

Finally, if $R$ has full row rank, i.e., $\operatorname{ker}_{D}(. R)=0$, then $T=L=\left(\begin{array}{ll}I_{r} & 0\end{array}\right) \in D^{r \times q}$ and thus $Q=D^{1 \times(q-r)}$, which yields the following extension of $D^{1 \times(q-r)}$ by $M$ :

$$
0 \longrightarrow D^{1 \times(q-r)} \stackrel{\alpha \circ \psi}{\longrightarrow} M_{1} \stackrel{\beta}{\longrightarrow} M \longrightarrow 0 .
$$

Finally, let us state a "dual version" of Example 1 .

Example 2. Let us split $R \in D^{q \times p}$ as $R=\left(\begin{array}{ll}R_{1} & R_{2}\end{array}\right)$, where $R_{1} \in D^{q \times r}$ and $R_{2} \in D^{q \times(p-r)}$, and let $Q_{1} \in D^{t \times q}$ be such that $\operatorname{ker}_{D}\left(. R_{1}\right)=D^{1 \times t} Q_{1}$. Repeating what we did previously for the left $D$-module module $N_{1}=D^{1 \times r} /\left(D^{1 \times q} R_{1}\right)$ (resp., $M=D^{1 \times p} /\left(D^{1 \times q} R\right)$ ) instead of $M$ (resp., $E)$, we get the short exact sequence

$$
0 \longrightarrow \operatorname{ker} \beta \stackrel{i}{\longrightarrow} M \stackrel{\beta}{\longrightarrow} N_{1} \longrightarrow 0,
$$

where $\beta\left(\pi\left(\lambda_{1} \quad \lambda_{2}\right)\right)=\kappa_{1}\left(\lambda_{1}\right)$ for all $\lambda_{1} \in D^{1 \times r}$ and all $\lambda_{2} \in D^{1 \times(p-r)}$ and $\kappa_{1}$ is the canonical projection onto $N_{1}$. Now, $m=\pi\left(\left(\begin{array}{ll}\lambda_{1} & \lambda_{2}\end{array}\right)\right) \in \operatorname{ker} \beta$ iff $\kappa_{1}\left(\lambda_{1}\right)=0$, i.e., iff there exists $\mu \in D^{1 \times q}$ such that $\lambda_{1}=\mu R_{1}$, i.e., $m=\pi\left(\left(\begin{array}{ll}\mu R_{1} & \lambda_{2}\end{array}\right)\right)$, which, using (9) of Lemma 11, shows that:

$$
\begin{aligned}
\operatorname{ker} \beta & =\left(D^{1 \times(q+p-r)}\left(\begin{array}{cc}
R_{1} & 0 \\
0 & I_{p-r}
\end{array}\right)\right) /\left(D^{1 \times q}\left(R_{1} \quad R_{2}\right)\right) \\
& \cong D^{1 \times(q+p-r)} /\left(D^{1 \times(q+t)}\left(\begin{array}{cc}
I_{q} & R_{2} \\
Q_{1} & 0
\end{array}\right)\right) \\
& \cong D^{1 \times(q+p-r)} /\left(D^{1 \times(q+t)}\left(\begin{array}{cc}
I_{q} & 0 \\
0 & Q_{1} R_{2}
\end{array}\right)\right) \\
& \cong D^{1 \times(p-r)} /\left(D^{1 \times t}\left(Q_{1} R_{2}\right)\right) .
\end{aligned}
$$

$\mathrm{RR} \mathrm{n}^{\circ} 7214$ 
If $L=D^{1 \times(p-r)} /\left(D^{1 \times t}\left(Q_{1} R_{2}\right)\right)$ is the left $D$-module finitely presented by $Q_{1} R_{2}$, then we get the extension $0 \longrightarrow L \stackrel{\alpha}{\longrightarrow} M \stackrel{\beta}{\longrightarrow} N_{1} \longrightarrow 0$ of $L$ by $N_{1}$, where $\alpha=i \circ \psi$ and $\psi: L \longrightarrow \operatorname{ker} \beta$ denotes the previous left $D$-isomorphism. Moreover, if $R_{1}$ has full row rank, i.e., $\operatorname{ker}_{D}\left(. R_{1}\right)=0$, then $L=D^{1 \times(p-r)}$ and $M$ defines an extension of $D^{1 \times(p-r)}$ by $N_{1}$.

\section{Serre's theorem}

Let us recall a few well-known definitions of module theory.

Definition $2\left([24,38)\right.$. Let $D$ be a left noetherian domain and $M=D^{1 \times p} /\left(D^{1 \times q} R\right)$ the left $D$-module finitely presented by the matrix $R \in D^{q \times p}$.

1. $M$ is free of rank $p \in \mathbb{N}=\{0,1, \ldots\}$ if $M \cong D^{1 \times p}$.

2. $M$ is stably free of rank $p-q$ if there exist two non-negative integers $p$ and $q$ such that $M \oplus D^{1 \times q} \cong D^{1 \times p}$, where $\oplus$ denotes the direct sum of left $D$-modules.

3. $M$ is projective if there exist a non-negative integer $p$ and a left $D$-module $P$ such that $M \oplus P \cong D^{1 \times p}$.

4. $M$ is torsion-free if the torsion left $D$-submodule

$$
t(M)=\{m \in M \mid \exists d \in D \backslash\{0\}: d m=0\}
$$

of $M$ is reduced to 0 , i.e., $t(M)=0$.

5. $M$ is torsion if $t(M)=M$, i.e., every $m \in M$ is a torsion element of $M$, namely, $m \in t(M)$.

6. $M$ is cyclic if $M$ is generated by one element $m \in M$, i.e., $M=D m \triangleq\{d m \mid d \in D\}$.

A free module is clearly stably free (take $q=0$ in 2 of Definition 2 ) and a stably free module is projective (take $P=D^{1 \times q}$ in 3 of Definition 2) and a projective module is torsion-free (since it can be embedded into a free, and thus, into a torsion-free module) but the converse of these results are generally not true for a general left noetherian domain $D$.

The next proposition characterizes stably free and free modules.

Proposition 1 ([6, 13, 33]). Let $D$ be a noetherian domain, $R \in D^{q \times p}$ a full row rank matrix, i.e., $\operatorname{ker}_{D}(. R)=0$, and the left $D$-module $M=D^{1 \times p} /\left(D^{1 \times q} R\right)$ finitely presented by $R$.

1. $M$ is a projective left $D$-module iff $M$ is a stably free left $D$-module.

2. $M$ is a stably free left $D$-module of rank $p-q$ iff $R$ admits a right-inverse over $D$, namely, iff there exists a matrix $S \in D^{p \times q}$ satisfying $R S=I_{q}$.

3. $M$ is a free left $D$-module of rank $p-q$ iff there exists $U \in \mathrm{GL}_{p}(D)$ such that:

$$
R U=\left(\begin{array}{ll}
I_{q} & 0
\end{array}\right) .
$$

If we write $U=\left(\begin{array}{ll}S \quad Q\end{array}\right)$, where $S \in D^{p \times q}$ and $Q \in D^{p \times(p-q)}$, then

$$
\begin{aligned}
\psi: M & \longrightarrow D^{1 \times(p-q)} \\
\pi(\lambda) & \longmapsto \lambda Q
\end{aligned}
$$


is a left D-isomorphism and its inverse $\psi^{-1}: D^{1 \times(p-q)} \longrightarrow M$ is defined by $\psi^{-1}(\mu)=$ $\pi(\mu T)$ for all $\mu \in D^{1 \times(p-q)}$, where the matrix $T \in D^{(p-q) \times p}$ is defined by:

$$
U^{-1}=\left(\begin{array}{c}
R \\
T
\end{array}\right) \in D^{p \times p} .
$$

Then, $M \cong D^{1 \times p} Q=D^{1 \times(p-q)}$ and the matrix $Q$ is called an injective parametrization of $M$. Finally, $\left\{\pi\left(T_{i} \bullet\right)\right\}_{i=1, \ldots, p-q}$ defines a basis of the free left $D$-module $M$ of rank $p-q$, where $T_{i}$ denotes the $i^{\text {th }}$ row of the matrix $T$.

When $D$ is a noncommutative polynomial ring over which Gröbner bases exist for any term order (e.g., certain classes of Ore algebras ([5])), constructive algorithms which check whether or not a finitely presented left $D$-module $M$ is torsion, torsion-free, projective or stably free are obtained in [6, 13, 33] by means of the computation of the left $D$-modules $\operatorname{ext}_{D}^{i}(N, D)$ for $i \in \mathbb{N}$ (see Remark 1). If $R$ has full row rank, then a right Gröbner basis computation can check whether or not $\operatorname{ext}_{D}^{1}(E, D)$ is reduced to 0 or equivalently check the existence of a right-inverse of $P=\left(\begin{array}{ll}R-\Lambda & -1\end{array}\right)$ over $D$. In [6, 33], we show how to compute the obstructions for a finitely presented left $D$-module to be stably free. These obstructions generate a two-sided ideal defined by the so-called $\pi$-polynomials. They can be used to characterize the obstructions for $\Lambda$ to define a stably free left $D$-module $E=D^{1 \times(p+q-r)} /\left(D^{1 \times q} P\right)$.

Checking whether or not $M$ is free is known to be a difficult problem in algebra. Let us state a few important results.

Theorem 3 ([19, 24, 36, 38]). We have the following results:

1. If $D$ is a principal left ideal domain, namely, every left ideal of $D$ can be generated by one element of $D$ (e.g., the ring of ordinary differential operators with coefficients in a differential field such that $K=\mathbb{R}$ or $\mathbb{Q}(t))$, then every torsion-free left D-module is free.

2. If $D=k\left[x_{1}, \ldots, x_{n}\right]$ is a commutative polynomial ring over a field $k$, then every projective $D$-module is free (Quillen-Suslin theorem).

3. If $k$ is a field of characteristic 0 (e.g., $\mathbb{Q}, \mathbb{R}, \mathbb{C}$ ) and $D=A_{n}(k)$ (resp., $B_{n}(k)$ ) is the first (resp., second) Weyl algebra of partial differential operators in $\partial_{1}=\frac{\partial}{\partial x_{1}}, \ldots, \partial_{n}=\frac{\partial}{\partial x_{n}}$ with coefficients in $k\left[x_{1}, \ldots, x_{n}\right]$ (resp., $k\left(x_{1}, \ldots, x_{n}\right)$ ), then every projective left $D$-module of rank at least 2 is free (Stafford's theorem).

4. If $D$ is the ring of ordinary differential operators with coefficients in the ring of formal power series $k \llbracket t \rrbracket$, where $k$ is a field of characteristic 0, or in the ring of convergent power series $k\{t\}$ with coefficients in $k=\mathbb{R}$ or $\mathbb{C}$, then every projective left D-module of rank at least 2 is free.

A natural question is whether or not there exists $\rho(\Lambda) \in \operatorname{ext}_{D}^{1}\left(M, D^{1 \times(q-r)}\right)$ such that the left $D$-module $E_{\rho(\Lambda)}=D^{1 \times(p+q-r)} /\left(D^{1 \times q} P\right)$ - finitely presented by the matrix $P=\left(\begin{array}{ll}R & -\Lambda\end{array}\right)$ and defining an extension of $D^{1 \times(q-r)}$ by $M$ - is respectively torsion-free, projective, stably free or free. In [39], J.-P. Serre studied when $E_{\rho(\Lambda)}$ is a projective or a free $D$-module when $M$ has a projective dimension equal to 1 (see, e.g., [38]) over a commutative polynomial ring $D=k\left[x_{1}, \ldots, x_{n}\right]$, where $k$ is a field. If the ring $D$ is strongly regular in the sense that every finitely generated left $D$-module admits a finite free resolution of finite length (see, e.g., [38]), then Serre's hypothesis is equivalent to the existence of a full row rank matrix $R \in D^{q \times p}$ 
satisfying $M \cong D^{1 \times p} /\left(D^{1 \times q} R\right)([33])$. See [33] for a constructive algorithm which computes such a matrix $R$ and [7] for its implementation in the package OreModules ([7]). In what follows, without loss of generality for the applications we have in mind (e.g., mathematical systems theory, control theory, mathematical physics), we shall assume that $M$ is finitely presented by a full row rank matrix $R \in D^{q \times p}$, i.e., $M=D^{1 \times p} /\left(D^{1 \times q} R\right)$ and $\operatorname{ker}_{D}(. R)=0$.

We recall that, by definition of the extension right $D$-module, we have:

$$
\operatorname{ext}_{D}^{1}(M, D)=D^{q} /\left(R D^{p}\right), \quad \operatorname{ext}_{D}^{1}(E, D)=D^{q} /\left(P D^{(p+q-r)}\right) .
$$

Now, using the following inclusions of right $D$-modules

$$
R D^{p} \subseteq P D^{(p+q-r)}=R D^{p}+\Lambda D^{(q-r)} \subseteq D^{q},
$$

Lemma 2 yields the following short exact sequence of right $D$-modules

$$
0 \longrightarrow\left(P D^{(p+q-r)}\right) /\left(R D^{p}\right) \stackrel{j}{\longrightarrow} \operatorname{ext}_{D}^{1}(M, D) \stackrel{\sigma}{\longrightarrow} \operatorname{ext}_{D}^{1}(E, D) \longrightarrow 0,
$$

where $j$ is the canonical injection. Hence, 12 shows that

$$
\begin{aligned}
\operatorname{ext}_{D}^{1}(E, D)=0 & \Leftrightarrow \operatorname{ext}_{D}^{1}(M, D)=\left(R D^{p}+\Lambda D^{(q-r)}\right) /\left(R D^{p}\right) \\
& \Leftrightarrow \operatorname{ext}_{D}^{1}(M, D)=\left(R D^{p}+\sum_{i=1}^{q-r} \Lambda_{\bullet} D\right) /\left(R D^{p}\right) \\
& \Leftrightarrow \quad \operatorname{ext}_{D}^{1}(M, D)=\sum_{i=1}^{q-r} \tau\left(\Lambda_{\bullet i}\right) D
\end{aligned}
$$

where $\tau: D^{p} \longrightarrow \operatorname{ext}_{D}^{1}(M, D)=D^{p} /\left(R D^{q}\right)$ is the canonical projection. Hence, $\operatorname{ext}_{D}^{1}(E, D)=0$ iff the right $D$-module $\operatorname{ext}_{D}^{1}(M, D)$ is generated by the family $\left\{\tau\left(\Lambda_{\bullet}\right)\right\}_{i=1, \ldots, q-r}$ of $q-r$ elements.

Lemma 3. With the previous notations, $\operatorname{ext}_{D}^{1}(E, D)=0$ iff the right $D$-module $\operatorname{ext}_{D}^{1}(M, D)$ is generated by $\left\{\tau\left(\Lambda_{\bullet}\right)\right\}_{i=1, \ldots, q-r}$, i.e., iff $\operatorname{ext}_{D}^{1}(M, D)$ can be generated by $q-r$ elements.

Let us now study the condition $\operatorname{ext}_{D}^{1}(E, D)=0$. By definition, $\operatorname{ext}_{D}^{1}(E, D)=0$ is equivalent to $D^{q}=P D^{(p+q-r)}$. If we denote by $\left\{g_{k}\right\}_{k=1, \ldots, q}$ the standard basis of $D^{q}$, then the last module equality is equivalent to the existence of a matrix $S_{k} \in D^{(p+q-r)}$ satisfying $g_{k}=P S_{k}$ for all $k=1, \ldots, q$, i.e., to the existence of the matrix $S=\left(S_{1} \ldots S_{q}\right) \in D^{(p+q-r) \times q}$ satisfying $P S=I_{q}$, i.e., a right-inverse of $P$ over $D$, which, by 2 of Proposition 1 , is equivalent to $E$ is a stably free left $D$-module.

Lemma 4. $\operatorname{ext}_{D}^{1}(E, D)=0$ iff the left $D$-module $E$ is stably free of rank $p-r$.

Similarly, $\operatorname{ext}_{D}^{1}(M, D)=0$ is equivalent to the existence of a right-inverse of the matrix $R$ over $D$, i.e., to $M$ is a stably free left $D$-module of rank $p-q$.

Combining Lemmas 3 and 4 , we obtain the following important result.

Theorem 4. Let $D$ be a noetherian domain, $R \in D^{q \times p}$ a full row rank matrix, namely, $\operatorname{ker}_{D}(. R)=0, \Lambda \in D^{q \times(q-r)}, P=\left(\begin{array}{ll}R & -\Lambda\end{array}\right) \in D^{q \times(p+q-r)}$ and $M=D^{1 \times p} /\left(D^{1 \times q} R\right)$ (resp., $\left.E=D^{1 \times(p+q-r)} /\left(D^{1 \times q} P\right)\right)$ the left $D$-module finitely presented by $R$ (resp., $\left.P\right)$ which defines the following extension of $D^{1 \times(q-r)}$ by $M$ :

$$
0 \longrightarrow D^{1 \times(q-r)} \stackrel{\alpha}{\longrightarrow} E \stackrel{\beta}{\longrightarrow} M \longrightarrow 0 .
$$

Then, the following results are equivalent: 
1. The left $D$-module $E$ is stably free of rank $p-r$.

2. The matrix $P=\left(\begin{array}{ll}R & -\Lambda\end{array}\right) \in D^{q \times(p+q-r)}$ admits a right-inverse over $D$.

3. $\operatorname{ext}_{D}^{1}(E, D)=D^{q} /\left(P D^{(p+q-r)}\right)=0$.

4. The right $D$-module $\operatorname{ext}_{D}^{1}(M, D)=D^{q} /\left(R D^{p}\right)$ is generated by $\left\{\tau\left(\Lambda_{\bullet}\right)\right\}_{i=1, \ldots, q-r}$, where $\tau: D^{q} \longrightarrow \operatorname{ext}_{D}^{1}(M, D)$ is the canonical projection onto $\operatorname{ext}_{D}^{1}(M, D)$.

Finally, the previous equivalences depend only on the residue class $\rho(\Lambda)$ of $\Lambda \in D^{q \times(q-r)}$ in the right D-module

$$
\operatorname{ext}_{D}^{1}\left(M, D^{1 \times(q-r)}\right)=D^{q \times(q-r)} /\left(R D^{p \times(q-r)}\right) \cong \operatorname{ext}_{D}^{1}(M, D)^{1 \times(q-r)},
$$

i.e., they depend only on the row vector $\left(\tau\left(\Lambda_{\bullet 1}\right) \ldots \tau\left(\Lambda_{\bullet}(q-r)\right)\right) \in \operatorname{ext}_{D}^{1}(M, D)^{1 \times(q-r)}$.

Remark 2. Theorem 4 was first obtained by J.-P. Serre in [39] for a commutative ring $D$ and $r=q-1$. In this case, $\operatorname{ext}_{D}^{1}(M, D)$ is the right $D$-module generated by $\tau(\Lambda)$, i.e., $\operatorname{ext}_{D}^{1}(M, D)$ is the cyclic right $D$-module generated by $\tau(\Lambda)$.

Example 3. Theorem 4 is fulfilled if $\operatorname{ext}_{D}^{1}(M, D)=0$, i.e., if $M$ is a stably free left $D$-module or, equivalently, if $R$ admits a right-inverse over $D$ since we can take $\Lambda=0$. Another explanation of this last result is that $\operatorname{ext}_{D}^{1}(M, D)$ is then the trivial cyclic left $D$-module or, equivalently, the short exact sequence 12 yields $\operatorname{ext}_{D}^{1}(E, D)=0$.

On simple examples over a commutative polynomial ring $D=k\left[x_{1}, \ldots, x_{n}\right]$ with coefficients in a computable field $k$ (e.g., $k=\mathbb{Q}$ or $\mathbb{F}_{p}$ where $p$ is a prime number), we can take a generic matrix $\Lambda \in D^{q \times(q-r)}$ with a fixed total degree in the $x_{i}$ 's and compute the $D$-module $\operatorname{ext}_{D}^{1}(E, D)=D^{1 \times q} /\left(D^{1 \times(p+q-r)} P^{T}\right)$ by means of a Gröbner basis computation and check whether or not the $D$-module $\operatorname{ext}_{D}^{1}(E, D)$ vanishes on certain branches of the corresponding tree of integrability conditions ([29]) or on certain parts of the underlying constellation of semialgebraic sets in the $k$-parameters of $\Lambda([21])$. See [21] for a survey explaining the constellation techniques and their implementations in Singular. In particular, we can test whether or not a non-zero constant belongs to the annihilator of the $D$-module $\operatorname{ext}_{D}^{1}(E, D)$, namely,

$$
\operatorname{ann}_{D}\left(\operatorname{ext}_{D}^{1}(E, D)\right)=\left\{d \in D \mid \forall n \in \operatorname{ext}_{D}^{1}(E, D), d n=0\right\}
$$

i.e., whether or not $\operatorname{ann}_{D}\left(\operatorname{ext}_{D}^{1}(E, D)\right)=D$. Since, $\operatorname{hom}_{D}\left(\operatorname{ext}_{D}^{1}(E, D), D\right) \cong \operatorname{ker}_{D}(. R)=0$ by Remark 1, $\operatorname{ext}_{D}^{1}(E, D)$ is a torsion right $D$-module (see Corollary 1 of [6]), and thus we obtain $\operatorname{ext}_{D}^{1}(E, D)=0$ iff $\operatorname{ann}_{D}\left(\operatorname{ext}_{D}^{1}(E, D)\right)=D$.

The constellation technique is particularly interesting when the finitely presented $D=$ $k\left[x_{1}, \ldots, x_{n}\right]$-module $\operatorname{ext}_{D}^{1}(M, D)$ is 0 -dimensional, i.e., when the ring $A=D / I$ is a finite $k$-vector space, where $I=\operatorname{ann}_{D}\left(\operatorname{ext}_{D}^{1}(M, D)\right)$. Indeed, a Gröbner basis computation of the $D$ module $R D^{p}$ then gives a finite set of row vectors $\left\{\lambda_{k}\right\}_{k=1, \ldots, s}$, where $\lambda_{k} \in D^{q}$ and $s=\operatorname{dim}_{k}(A)$, such that $\operatorname{ext}_{D}^{1}(M, D)=\bigoplus_{k=1}^{s} k \tau\left(\lambda_{k}\right)$. Then, we can consider a generic matrix of the form

$$
\Lambda=\left(\begin{array}{lll}
\sum_{k=1}^{s} a_{1 k} \lambda_{k} & \ldots & \sum_{k=1}^{s} a_{(q-r) k} \lambda_{k}
\end{array}\right) \in D^{q \times(q-r)},
$$

where the $a_{l k}$ 's are arbitrary elements of $k$ for $l=1, \ldots,(q-r)$ and $k=1, \ldots, s$, and compute the constellation of semi-algebraic sets corresponding to the possible vanishing of $\operatorname{ext}_{D}^{1}(E, D)$. 
Example 4. We consider the model of a string with an interior mass defined by

$$
\left\{\begin{array}{l}
\phi_{1}(t)+\psi_{1}(t)-\phi_{2}(t)-\psi_{2}(t)=0 \\
\dot{\phi}_{1}(t)+\dot{\psi}_{1}(t)+\eta_{1} \phi_{1}(t)-\eta_{1} \psi_{1}(t)-\eta_{2} \phi_{2}(t)+\eta_{2} \psi_{2}(t)=0 \\
\phi_{1}\left(t-2 h_{1}\right)+\psi_{1}(t)-u\left(t-h_{1}\right)=0 \\
\phi_{2}(t)+\psi_{2}\left(t-2 h_{2}\right)-v\left(t-h_{2}\right)=0
\end{array}\right.
$$

introduced and studied in [25], where $h_{1}, h_{2} \in \mathbb{R}_{+}$are such that $\mathbb{Q} h_{1}+\mathbb{Q} h_{2}$ is a 2-dimensional $\mathbb{Q}$ vector space and $\eta_{1}$ and $\eta_{2}$ are two constant parameters. Let us denote by $D=\mathbb{Q}\left(\eta_{1}, \eta_{2}\right)\left[\partial, \sigma_{1}, \sigma_{2}\right]$ the commutative polynomial algebra of differential incommensurable time-delay operators in $\partial$, $\sigma_{1}$ and $\sigma_{2}$, where:

$$
\partial f(t)=\dot{f}(t), \quad \sigma_{1} f(t)=f\left(t-h_{1}\right), \quad \sigma_{2} f(t)=f\left(t-h_{2}\right) .
$$

Let $M=D^{1 \times 6} /\left(D^{1 \times 4} R\right)$ be the $D$-module finitely presented by the matrix

$$
R=\left(\begin{array}{cccccc}
1 & 1 & -1 & -1 & 0 & 0 \\
\partial+\eta_{1} & \partial-\eta_{1} & -\eta_{2} & \eta_{2} & 0 & 0 \\
\sigma_{1}^{2} & 1 & 0 & 0 & -\sigma_{1} & 0 \\
0 & 0 & 1 & \sigma_{2}^{2} & 0 & -\sigma_{2}
\end{array}\right) \in D^{4 \times 6}
$$

which is associated with $(13)$. Then, we have $\operatorname{ext}_{D}^{1}(M, D)=D^{4} /\left(R D^{6}\right)$. Computing a Gröbner basis of the $D$-module $\operatorname{ext}_{D}^{1}(M, D)$, we obtain that $\operatorname{ext}_{D}^{1}(M, D)$ is a 1-dimensional $\mathbb{Q}\left(\eta_{1}, \eta_{2}\right)$ vector space and $\tau\left(\left(\begin{array}{llll}0 & 0 & 0 & 1\end{array}\right)^{T}\right)$ is a basis, where $\tau: D^{4} \longrightarrow \operatorname{ext}_{D}^{1}(M, D)$ is the canonical projection onto $\operatorname{ext}_{D}^{1}(M, D)$. Hence, the only possible $\Lambda$ 's for which $P=(R-\Lambda)$ admits a rightinverse over $D$ belong to $\left.V=\left\{\begin{array}{llll}a(0 & 0 & 0 & 1\end{array}\right)^{T} \mid a \in \mathbb{Q}\left(\eta_{1}, \eta_{2}\right)\right\}$. In particular, if we consider the column vector $\Lambda=\left(\begin{array}{llll}0 & 0 & 0 & 1\end{array}\right)^{T}$, then we can easily check that $P=\left(\begin{array}{ll}R & -\Lambda\end{array}\right) \in D^{4 \times 7}$ admits the following right-inverse over $D$ :

$$
S=\left(\begin{array}{ccccccc}
0 & 0 & -\frac{1}{2} & -\frac{1}{2} & 0 & -\frac{1}{2} \sigma_{2} & -\frac{1}{2} \\
0 & 0 & -\frac{1}{2 \eta_{2}} & \frac{1}{2 \eta_{2}} & 0 & \frac{1}{2 \eta_{2}} \sigma_{2} & -\frac{1}{2 \eta_{2}} \\
-1 & 1 & -\frac{\eta_{1}}{\eta_{2}} & \frac{\eta_{1}}{\eta_{2}} & -\sigma_{1} & \frac{\eta_{1}}{\eta_{2}} \sigma_{2} & -\frac{\eta_{1}}{\eta_{2}} \\
0 & 0 & 0 & 0 & 0 & 0 & -1
\end{array}\right)^{T}
$$

Hence, the $D$-module $\operatorname{ext}_{D}^{1}(M, D)=D^{4} /\left(R D^{6}\right)$ is cyclic and is generated by $\tau(\Lambda)$.

Remark 3. If $D=k\left[x_{1}, x_{2}\right]$ is a commutative polynomial ring over a field $k, R \in D^{q \times p}$ and $M=D^{1 \times p} /\left(D^{1 \times q} R\right)$, then $M$ can either admit a non-trivial torsion submodule $t(M)$, be torsion-free or projective, i.e., free by the Quillen-Suslin. For more details, see, e.g., [6, 27, 28]. Hence, if $p>q$ and $R$ has full row rank, then the generic situation is that $M$ is a torsion-free $D$-module, which implies that $\operatorname{ext}_{D}^{1}(M, D)$ is generically 0 -dimensional since $D$ is a so-called regular Cohen-Macaulay ring (see, e.g., [12]). Indeed, we then have that the codimension of $\operatorname{ext}_{D}^{1}(M, D)$, namely, $2-\operatorname{dim}_{D}\left(\operatorname{ext}_{D}^{1}(M, D)\right)$, where $\operatorname{dim}_{D}\left(\operatorname{ext}_{D}^{1}(M, D)\right)$ denotes the so-called Krull dimension of the ring $A=D / I$, where $I$ is the annihilator of $\operatorname{ext}_{D}^{1}(M, D)$ (see, e.g., [38]), is equal to the grade $j_{D}\left(\operatorname{ext}_{D}^{1}(M, D)\right)$ of $\operatorname{ext}_{D}^{1}(M, D)$, namely, 2 if $M$ is torsion-free but not free and 3 if $M$ is projective $\left(\operatorname{dim}\left(\operatorname{ext}_{D}^{1}(M, D)\right)=-1\right.$ means that $\left.\operatorname{ext}_{D}^{1}(M, D)=0\right)$. For more 
details, see [28] and the references therein. Hence, using constellation techniques, we can check whether or not there exists a matrix $\Lambda \in D^{q \times(q-r)}$ such that $P=\left(\begin{array}{ll}R & -\Lambda\end{array}\right)$ admits a rightinverse over $D=k\left[x_{1}, x_{2}\right]$, whenever $R$ is a generic full row rank matrix with $p>q$ and the columns of the matrix $\Lambda$ are generic $k$-linear combinations of the basis of the finite-dimensional $k$-vector $\operatorname{ext}_{D}^{1}(M, D)$. This situation particularly holds in the study of control linear differential time-delay systems defined over $D=k[\partial, \delta]$. For more details, see [6, 7, $\underline{8}, \mathbf{9}$.

Apart from the previous 0-dimensional case, we do not know yet how to recognize the existence of $\Lambda \in D^{q \times(q-r)}$ satisfying 2 of Theorem 4. However, using an ansatz, we can give the sketch of an algorithm in the case of the second Weyl algebra $B_{n}(k)$. This case encapsulates the cases of a commutative polynomial ring and the first Weyl algebra $A_{n}(k)$ since we have:

$$
k\left[x_{1}, \ldots, x_{n}\right] \subset A_{n}(k) \subset B_{n}(k) .
$$

Algorithm 1. - Input: Let $k$ be an algebraically closed computational field, $D=B_{n}(k)$, $R \in D^{q \times p}$ a full row rank matrix and three non-negative integers $\alpha, \beta$ and $\gamma$.

- Output: A set (possibly empty) of $\left\{\Lambda_{i}\right\}_{i \in I}$ such that the matrix $\left(R-\Lambda_{i}\right)$ admits a right-inverse over $D$.

1. Consider an ansatz $\Lambda \in D^{q \times(q-r)}$ whose entries have a fixed total order $\alpha$ in the $\partial_{i}$ 's and a fixed total degree $\beta$ (resp., $\gamma$ ) for the polynomial numerators (resp., denominators) in the $x_{j}$ 's of the arbitrary coefficients of the ansatz $\Lambda$.

2. Compute a Gröbner basis of the right $D$-module $R D^{p}$.

3. Compute the normal form $\bar{\Lambda}_{\bullet} i \in D^{q}$ of the $i^{\text {th }}$ column $\Lambda_{\bullet}$ of $\Lambda$ in the right $D$-module $\operatorname{ext}_{D}^{1}(M, D)=D^{q} /\left(R D^{p}\right)$ for all $i=1, \ldots, q-r$.

4. Compute the obstructions for projectivity of the left $D$-module

$$
\bar{E}=D^{1 \times(p+q-r)} /\left(D^{1 \times q}(R-\bar{\Lambda})\right)
$$

(e.g., computation of a Gröbner basis of the right $D$-module $(R-\bar{\Lambda}) D^{(p+q-r)}$ or computation of the $\pi$-polynomials of the left $D$-module $\bar{E}$ ).

5. Solve the systems in the arbitrary coefficients of the ansatz $\Lambda$ obtained by making the obstructions vanish.

6. Return the set of solutions for $\Lambda$.

Let us illustrate Algorithm 1 with explicit examples.

Example 5. Let us consider a general transmission line defined by

$$
\left\{\begin{array}{l}
\frac{\partial V}{\partial x}+L \frac{\partial I}{\partial t}+R I=0 \\
C \frac{\partial V}{\partial t}+G V+\frac{\partial I}{\partial x}=0
\end{array}\right.
$$

where $I$ denotes the current, $V$ the voltage, $L$ the self-inductance, $R$ the resistance, $C$ the capacitor and $G$ the conductance. Let $D=\mathbb{Q}(L, R, C, G)\left[\partial_{t}, \partial_{x}\right]$ be the commutative polynomial 
ring of partial differential operators in $\partial_{t}$ and $\partial_{x}$ with coefficients in the field $\mathbb{Q}(L, R, C, G)$, the presentation matrix $J \in D^{2 \times 2}$ of $(14)$ defined by

$$
J=\left(\begin{array}{cc}
\partial_{x} & L \partial_{t}+R \\
C \partial_{t}+G & \partial_{x}
\end{array}\right)
$$

and the $D$-module $M=D^{1 \times 2} /\left(D^{1 \times 2} J\right)$. In this example, we have slightly changed the previous notations since the standard notation for a resistance is also $R$. Let us consider $\Lambda=\left(\begin{array}{ll}\alpha & \beta\end{array}\right)^{T}$, where $\alpha$ and $\beta$ are two new variables, $A=D[\alpha, \beta], P=\left(\begin{array}{ll}J & -\Lambda\end{array}\right) \in A^{2 \times 3}$ and the $A$-module $E=A^{1 \times 3} /\left(A^{1 \times 2} P\right)$ finitely presented by $P$. Using the results developed in [6] and the package OreModules ([7]), the obstructions for $E$ to be a stably free $A$-module are defined by $A /\left(\pi_{1}, \pi_{2}\right)$, where the $\pi$-polynomials $\pi_{1}$ and $\pi_{2}$ are respectively:

$$
\left\{\begin{array}{l}
\pi_{1}=\left(C \alpha^{2}-L \beta^{2}\right) \partial_{t}+G \alpha^{2}-R \beta^{2} \\
\pi_{2}=\left(C \alpha^{2}-L \beta^{2}\right) \partial_{x}+(L G-R C) \alpha \beta
\end{array}\right.
$$

Hence, if $C \alpha^{2}=L \beta^{2}$ and $G \alpha^{2}-R \beta^{2} \neq 0$ (resp., $\left.(L G-R C) \alpha \beta \neq 0\right)$, then $\pi_{1}$ (resp., $\pi_{2}$ ) is a non-zero constant. In particular, if we consider the following values

$$
\beta=C \neq 0, \quad \alpha^{2}=L C \neq 0, \quad L G-R C \neq 0,
$$

the ring $B=\left(\mathbb{Q}(L, R, C, G)[\alpha] /\left(\alpha^{2}-L C\right)\right)\left[\partial_{t}, \partial_{x}\right]$ and $\Lambda=\left(\begin{array}{ll}\alpha & C\end{array}\right)^{T} \in B^{2}$, then the matrix $P=\left(\begin{array}{ll}J & -\Lambda\end{array}\right) \in B^{2 \times 3}$ admits the following right-inverse over $B$ :

$$
S=\frac{1}{(R C-L G)}\left(\begin{array}{cc}
-\alpha & L \\
-C & \alpha \\
-\left(C \partial_{x}+\alpha C \partial_{t}+\alpha G\right) / C & \left(\alpha \partial_{x}+L C \partial_{t}+R C\right) / C
\end{array}\right) .
$$

Therefore, the $B$-module $\operatorname{ext}_{B}^{1}(M, B)=B^{2} /\left(J B^{3}\right)$ is cyclic and is generated by $\tau(\Lambda)$, where $\tau: B^{2} \longrightarrow \operatorname{ext}_{B}^{1}(M, B)$ is the canonical projection onto $\operatorname{ext}_{B}^{1}(M, B)$.

Remark 4. If $D$ admits an involution $\theta$, namely, an anti-isomorphism of $D$ of order two or, equivalently, $\theta: D \longrightarrow D$ satisfies that $\theta^{2}=\operatorname{id}_{D}, \theta\left(d+d^{\prime}\right)=\theta(d)+\theta\left(d^{\prime}\right)$ and $\theta\left(d d^{\prime}\right)=\theta\left(d^{\prime}\right) \theta(d)$, for all $d$ and $d^{\prime} \in D$, and if we introduce $\theta(P)=\left(\theta\left(P_{i j}\right)\right)^{T} \in D^{(p+q-r) \times q}$, then the assertions of Theorem 4 are equivalent to $D^{1 \times q} /\left(D^{1 \times(p+q-r)} \theta(R)\right)=0$, which is also equivalent to the existence of a left-inverse of $\theta(R)$ over $D$. For more details, see [6].

Example 6. Let us consider the conjugate Beltrami equations with $\sigma=x^{-1}$ :

$$
\left\{\begin{array}{l}
\frac{\partial u}{\partial x}-x \frac{\partial v}{\partial y}=0 \\
\frac{\partial u}{\partial y}+x \frac{\partial v}{\partial x}=0
\end{array}\right.
$$

Let $D=A_{2}(\mathbb{Q}(a, b))=\mathbb{Q}(a, b)[x, y]\left\langle\partial_{x}, \partial_{y}\right\rangle$ be the ring of noncommutative polynomials in $\partial_{x}$, $\partial_{y}, x$ and $y$ with coefficients in the field $\mathbb{Q}(a, b)$ which satisfy

$$
x y=y x, \quad \partial_{x} \partial_{y}=\partial_{y} \partial_{x}, \quad \partial_{x} x=x \partial_{x}+1, \quad \partial_{y} y=y \partial_{y}+1,
$$

the presentation matrix $R \in D^{2 \times 2}$ of 16 defined by

$$
R=\left(\begin{array}{cc}
\partial_{x} & -x \partial_{y} \\
\partial_{y} & x \partial_{x}
\end{array}\right)
$$

RR $\mathrm{n}^{\circ} 7214$ 
and the left $D$-module $M=D^{1 \times 2} /\left(D^{1 \times 2} R\right)$ finitely presented by $R$. If we consider the column vector $\Lambda=\left(\begin{array}{ll}a & b\end{array}\right)^{T}$ and the left $D$-module $E=D^{1 \times 3} /\left(D^{1 \times 2} P\right)$ finitely presented by the matrix $P=\left(\begin{array}{ll}R & -\Lambda\end{array}\right) \in D^{2 \times 3}$, then we can check that $P$ admits the following right-inverse over $D$ whenever both $a$ and $b$ are non-zero:

$$
S=\left(\begin{array}{cc}
x\left(a x \partial_{x}+b x \partial_{y}+a\right) / a & -x\left(a x \partial_{x}+b x \partial_{y}+a\right) / b \\
-\left(a x \partial_{y}-b x \partial_{x}-2 b\right) / a & \left(a x \partial_{y}-b x \partial_{x}-2 b\right) / b \\
x\left(x \partial_{x}^{2}+x \partial_{y}^{2}+3 \partial_{x}\right) / a & -\left(x^{2} \partial_{x}^{2}+x^{2} \partial_{y}^{2}+3 x \partial_{x}+1\right) / b
\end{array}\right) \in D^{3 \times 2}
$$

Hence, the right $D$-module $\operatorname{ext}_{D}^{1}(M, D)=D^{2} /\left(R D^{3}\right)$ is cyclic and is generated by $\tau(\Lambda)$, where $\tau: D^{2} \longrightarrow \operatorname{ext}_{D}^{1}(M, D)$ is the canonical projection onto $\operatorname{ext}_{D}^{1}(M, D)$.

We have the following corollary of Theorem 4 .

Corollary 1. Let $A$ be a commutative ring, $D=A[x], F \in A^{p \times p}, R=x I_{p}-F \in D^{p \times p}$ and $M=D^{1 \times p} /\left(D^{1 \times p} R\right)$. Then, the following assertions are equivalent:

1. There exists $\Lambda \in D^{p \times(p-r)}$ such that the $D$-module $E=D^{1 \times(2 p-r)} /\left(D^{1 \times p}(R-\Lambda)\right)$ is stably free of rank $p-r$.

2. $\left\{\tau\left(\Lambda_{\bullet}\right)\right\}_{i=1, \ldots, p-r}$ generates the following finitely presented $D$-module:

$$
\operatorname{ext}_{D}^{1}(M, D)=D^{p} /\left(R D^{p}\right) \cong D^{1 \times p} /\left(D^{1 \times p} R^{T}\right) .
$$

3. There exists a matrix $\bar{\Lambda} \in A^{p \times(p-r)}$ such that the matrix

$$
\Omega \triangleq\left(\begin{array}{llll}
\bar{\Lambda} & F \bar{\Lambda} & F^{2} \bar{\Lambda} & \ldots
\end{array} F^{p-1} \bar{\Lambda}\right) \in A^{p \times p(p-r)}
$$

admits a right-inverse over $A$.

In particular, if $\bar{\Lambda} \in A^{p \times(p-r)}$ satisfies 2 then $\Lambda=\bar{\Lambda}$ satisfies 1 .

Proof. 1 and 2 are equivalent by Theorem 4. Moreover, Theorem 4 shows that the property of $E$ of being a stably free $D$-module depends only on the residue classes $\left\{\tau\left(\Lambda_{\bullet i}\right)\right\}_{i=1, \ldots, p-r}$ of the columns $\left\{\Lambda_{\bullet}\right\}_{i=1, \ldots, p-r}$ of $\Lambda$ in the $D$-module $\operatorname{ext}_{D}^{1}(M, D)=D^{p} /\left(R D^{p}\right)$. Since $R=x I_{p}-F$ and $F \in A^{p \times p}$, we can assume without loss of generality that $\Lambda_{\bullet} \in A^{p}$ for all $i=1, \ldots, p-r$, i.e., $\Lambda \in A^{p \times(p-r)}$. If $P=(R-\Lambda)$ then $\operatorname{ext}_{D}^{1}(E, D)=D^{p} /\left(P D^{2 p-r}\right) \cong D^{1 \times p} /\left(D^{1 \times(2 p-r)} P^{T}\right)$ is defined by the generators $\left\{y_{j}\right\}_{j=1, \ldots, p}$ which satisfy the following $D$-linear relations

$$
\left\{\begin{array}{l}
x y-F^{T} y=0 \\
\Lambda^{T} y=0
\end{array}\right.
$$

where $y=\left(\begin{array}{lll}y_{1} & \ldots & y_{p}\end{array}\right)^{T}$. Multiplying the last equation by $x$ and using the first one, we obtain the new equation $\Lambda^{T} F^{T} y=0$. Repeating inductively the same operations, we get $\Lambda^{T} F^{T^{k}} y=0$ for all $k \in \mathbb{N}$. Using the Caley-Hamilton theorem stating that $F^{p}=\sum_{k=0}^{p-1} a_{k} F^{k}$ for certain $a_{k}$ 's in $A$ (see, e.g., [12]), we obtain:




By Theorem 4, $E$ is a stably free of rank $p-r$ iff $\operatorname{ext}_{D}^{1}(E, D)=0$, i.e., iff 119 is equivalent to $y=0$, i.e., iff $\left(\begin{array}{llll}\Lambda & F \Lambda & \ldots & F^{p-1} \Lambda\end{array}\right)^{T}$ admits a left-inverse over $A$, i.e., iff the matrix $\Omega=\left(\begin{array}{llll}\Lambda & F \Lambda & \ldots & F^{p-1} \Lambda\end{array}\right)$ admits a right-inverse over $A$.

Corollary 1 is a generalization of a result of [20]. See [10] for an extension.

Example 7. We note that (15) has the form of $J=\partial_{x} I_{2}-F \in D^{2 \times 2}$, where

$$
F=-\left(\begin{array}{cc}
0 & L \partial_{t}+R \\
C \partial_{t}+G & 0
\end{array}\right) \in \mathbb{Q}(L, R, C, G)\left[\partial_{t}\right]^{2 \times 2},
$$

and $D=A\left[\partial_{x}\right]$. If $\bar{\Lambda} \in A^{2}$, then the matrix $\Omega$ defined by $(18)$ is

$$
\Omega=\left(\begin{array}{ll}
\bar{\Lambda}_{1} & -\left(L \partial_{t}+R\right) \bar{\Lambda}_{2} \\
\bar{\Lambda}_{2} & -\left(C \partial_{t}+G\right) \bar{\Lambda}_{1}
\end{array}\right)
$$

and thus $\operatorname{det} \Omega=-\left(C \partial_{t}+G\right) \bar{\Lambda}_{1}^{2}+\left(L \partial_{t}+R\right) \bar{\Lambda}_{2}^{2}=\left(L \bar{\Lambda}_{2}^{2}-C \bar{\Lambda}_{1}^{2}\right) \partial_{t}+\left(R \bar{\Lambda}_{2}^{2}-G \bar{\Lambda}_{1}^{2}\right)$. Thus, $\operatorname{det} \Omega \in k \backslash\{0\}$ iff $L \bar{\Lambda}_{2}^{2}-C \bar{\Lambda}_{1}^{2}=0$ and $R \bar{\Lambda}_{2}^{2}-G \bar{\Lambda}_{1}^{2} \in k \backslash\{0\}$. In particular, if $\bar{\Lambda}_{1}, \bar{\Lambda}_{2} \in k$, then $\operatorname{det} \Omega \in k \backslash\{0\}$ iff $L \bar{\Lambda}_{2}^{2}-C \bar{\Lambda}_{1}^{2}=0$ and $R \bar{\Lambda}_{2}^{2}-G \bar{\Lambda}_{1}^{2} \neq 0$ and we find again the result obtained in Example 5 .

Example 8. Let $k$ be a field, $A=k[y], D=A[x]=k[x, y]$ and the matrices

$$
F=\left(\begin{array}{cc}
0 & y+1 \\
y^{2} & 0
\end{array}\right) \in A^{2 \times 2}, \quad R=x I_{2}-F=\left(\begin{array}{cc}
x & -y-1 \\
-y^{2} & x
\end{array}\right) \in D^{2 \times 2}
$$

considered in [20]. Let $M=D^{1 \times 2} /\left(D^{1 \times 2} R\right)$ be the $D$-module finitely presented by $R$ and $\bar{\Lambda} \in A^{2}$ a column vector. Then, using $(18)$, the matrix $\Omega$ is defined by:

$$
\Omega=\left(\begin{array}{cc}
\bar{\Lambda}_{1} & (y+1) \bar{\Lambda}_{2} \\
\bar{\Lambda}_{2} & y^{2} \bar{\Lambda}_{1}
\end{array}\right) .
$$

Since $\operatorname{det} \Omega=\left(y \bar{\Lambda}_{1}\right)^{2}-(y+1) \bar{\Lambda}_{2}^{2}$ has degree at least 1 in $y$, we get $\operatorname{det} \Omega \notin k \backslash\{0\}$ for all $\bar{\Lambda} \in A^{2}$, and thus, by Corollary 1 , there exists no column vector $\Lambda \in D^{2}$ such that the $D$-module $E=D^{1 \times 3} /\left(D^{1 \times 2}(R-\Lambda)\right)$ is stably free.

Let us now consider the following matrices

$$
F^{\prime}=\left(\begin{array}{cc}
0 & 1 \\
y^{2}(y+1) & 0
\end{array}\right) \in A^{2 \times 2}, \quad R^{\prime}=x I_{2}-F^{\prime}=\left(\begin{array}{cc}
x & -1 \\
-y^{2}(y+1) & x
\end{array}\right),
$$

also considered in [20], the $D$-module $M^{\prime}=D^{1 \times 2} /\left(D^{1 \times 2} R^{\prime}\right)$ finitely presented by $R^{\prime}$ and $\bar{\Lambda} \in A^{2}$. The matrix $\Omega^{\prime}$ defined by $(18)$ is then

$$
\Omega^{\prime}=\left(\begin{array}{cc}
\bar{\Lambda}_{1} & \bar{\Lambda}_{2} \\
\bar{\Lambda}_{2} & y^{2}(y+1) \bar{\Lambda}_{1}
\end{array}\right)
$$

and $\operatorname{det} \Omega^{\prime}=y^{2}(y+1) \bar{\Lambda}_{1}-\bar{\Lambda}_{2}^{2}$. Hence, if we consider $\bar{\Lambda}_{1}=0$ and $\bar{\Lambda}_{2}=1$, then $\operatorname{det} \Omega^{\prime}=$ $-1 \in k \backslash\{0\}$, which, by Corollary 1 , proves that the $D$-module $E^{\prime}=D^{1 \times 3} /\left(D^{1 \times 2}\left(R^{\prime}-\bar{\Lambda}\right)\right)$ is a stably free, and thus free of rank 1 by the Quillen-Suslin theorem (see 2 of Theorem 3 ). Finally, 4 of Theorem 4 shows that $\operatorname{ext}_{D}^{1}(M, D)$ is not a cyclic $D$-module whereas the $D$-module $\operatorname{ext}_{D}^{1}\left(M^{\prime}, D\right)$ is cyclic, a fact implying that the $D$-modules $M$ and $M^{\prime}$ are not isomorphic. This result is not trivial since the main basic invariants of $M$ and $M^{\prime}$ are the same. 


\section{Serre's reduction problem}

We can now use Theorem 4 to study Serre's reduction.

Theorem 5. Let $D$ be a noetherian domain, $R \in D^{q \times p}$ a full row rank matrix, $0 \leq r \leq q-1$ and a matrix $\Lambda \in D^{q \times(q-r)}$ such that there exists $U \in \mathrm{GL}_{p+q-r}(D)$ satisfying:

$$
\left(\begin{array}{ll}
R & -\Lambda
\end{array}\right) U=\left(\begin{array}{ll}
I_{q} & 0
\end{array}\right) .
$$

If we decompose the unimodular matrix $U$ as follows

$$
U=\left(\begin{array}{ll}
S_{1} & Q_{1} \\
S_{2} & Q_{2}
\end{array}\right),
$$

where $S_{1} \in D^{p \times q}, S_{2} \in D^{(q-r) \times q}, Q_{1} \in D^{p \times(p-r)}, Q_{2} \in D^{(q-r) \times(p-r)}$, and if we introduce the left $D$-module $L=D^{1 \times(p-r)} /\left(D^{1 \times(q-r)} Q_{2}\right)$ finitely presented by the full row rank matrix $Q_{2}$, i.e., defined by the following short exact sequence

$$
0 \longrightarrow D^{1 \times(q-r)} \stackrel{\cdot Q_{2}}{\longrightarrow} D^{1 \times(p-r)} \stackrel{\kappa}{\longrightarrow} L \longrightarrow 0,
$$

then we have:

$$
M=D^{1 \times p} /\left(D^{1 \times q} R\right) \cong L=D^{1 \times(p-r)} /\left(D^{1 \times(q-r)} Q_{2}\right) .
$$

Conversely, if $M$ is isomorphic to a left $D$-module $L$ defined by the short exact sequence (22) for a certain matrix $Q_{2} \in D^{(q-r) \times(p-r)}$, then there exist $\Lambda \in D^{q \times(q-r)}$ and $U \in \mathrm{GL}_{p+q-r}(D)$ such that $(R-\Lambda) U=\left(\begin{array}{ll}I_{q} & 0\end{array}\right)$.

Proof. $\Rightarrow$ By hypothesis, we have $\left(\begin{array}{ll}R & -\Lambda\end{array}\right) S=I_{q}$, where $S=\left(\begin{array}{ll}S_{1}^{T} & S_{2}^{T}\end{array}\right)^{T}$, which shows that $P=\left(\begin{array}{ll}R & -\Lambda\end{array}\right)$ admits a right-inverse over $D$. By Theorem 4 , the extension $(4)$ of $D^{1 \times(q-r)}$ by $M$ is then defined by a stably free left $D$-module $E$, and thus, free of rank $p-r$ by 3 of Proposition 1 applied to $E$. Moreover, by 3 of Proposition 1, the left $D$-homomorphism $\psi: E \longrightarrow D^{1 \times(p-r)}$ defined by $\psi\left(\varrho\left(\left(\mu_{1} \quad \mu_{2}\right)\right)\right)=\mu_{1} Q_{1}+\mu_{2} Q_{2}$ for all $\mu_{1} \in D^{1 \times p}$ and all $\mu_{2} \in D^{1 \times(q-r)}$, is a left $D$-isomorphism, which yields the equivalence of extensions of $D^{1 \times(q-r)}$ by $M$ :

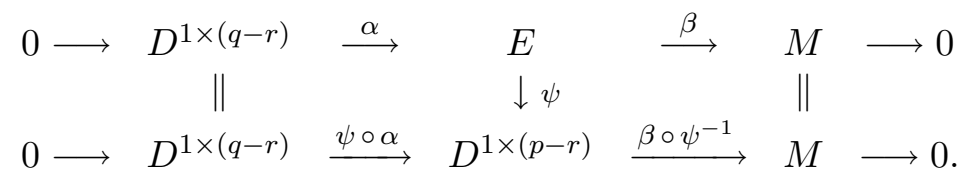

Using the standard basis $\left\{e_{i}\right\}_{i=1, \ldots, q-r}$ of $D^{1 \times(q-r)}$, we obtain

$$
\forall i=1, \ldots, q-r, \quad(\psi \circ \alpha)\left(e_{i}\right)=\psi\left(\alpha\left(e_{i}\right)\right)=\psi\left(\varrho\left(\left(\begin{array}{ll}
0 & e_{i}
\end{array}\right)\right)=e_{i} Q_{2},\right.
$$

which implies that $\psi \circ \alpha: D^{1 \times(q-r)} \longrightarrow D^{1 \times(p-r)}$ is defined by $(\psi \circ \alpha)(\nu)=\nu Q_{2}$ for all $\nu \in D^{1 \times(q-r)}$. The matrix $Q_{2}$ has full row rank since $\psi \circ \alpha$ is injective as the composition of two injective left $D$-homomorphisms. If $L=D^{1 \times(p-r)} /\left(D^{1 \times(q-r)} Q_{2}\right)$ is the left $D$-module finitely presented by the matrix $Q_{2} \in D^{(q-r) \times(p-r)}$ and $\kappa: D^{1 \times(p-r)} \longrightarrow L$ the canonical projection onto $L$, then we get 22$)$ and $L=\operatorname{coker}_{D}\left(. Q_{2}\right) \cong \operatorname{im}\left(\beta \circ \psi^{-1}\right)=M$.

$\Leftarrow$ Let us suppose that there exists a left $D$-isomorphism $\gamma: L \longrightarrow M$, where $L$ is defined by 22 . Then, we have the following extension of $D^{1 \times(q-r)}$ by $M$ :

$$
0 \longrightarrow D^{1 \times(q-r)} \stackrel{. Q_{2}}{\longrightarrow} D^{1 \times(p-r)} \stackrel{\gamma \circ \kappa}{\longrightarrow} M \longrightarrow 0 .
$$


By Theorem 2, the equivalence class of extension (24) defines a unique element $\rho(\Lambda)$ of the right $D$-module $\operatorname{ext}_{D}\left(M, D^{1 \times(q-r)}\right)$ for a certain matrix $\Lambda \in D^{q \times(q-r)}$. Then, the finitely presented left $D$-module $E=D^{1 \times(p+q-r)} /\left(D^{1 \times q}(R \quad-\Lambda)\right)$ defines the extension (4) of $D^{1 \times(q-r)}$ by $M$ which belongs to the same equivalence class as $(24)$. Since extensions of $D^{1 \times(q-r)}$ by $M$ belonging to the same equivalence class are defined by isomorphic central left $D$-modules (see the comment after Definition 1), we obtain $E \cong D^{1 \times(p-r)}$. Hence, $E$ is a free left $D$-module of rank $p-r$, which, by 2 of Proposition 1 , implies the existence a matrix $U \in \mathrm{GL}_{p+q-r}(D)$ such that:

$$
\left(\begin{array}{ll}
R & -\Lambda
\end{array}\right) U=\left(\begin{array}{ll}
I_{q} & 0
\end{array}\right) .
$$

We now can give an explicit description of the isomorphism obtained in Theorem 5 .

Corollary 2. With the notations of Theorem 5, the left D-isomorphism (23) obtained in Theorem 5 is explicitly defined by:

$$
\begin{aligned}
\varphi: M=D^{1 \times p} /\left(D^{1 \times q} R\right) & \longrightarrow L=D^{1 \times(p-r)} /\left(D^{1 \times(q-r)} Q_{2}\right) \\
\pi(\lambda) & \longmapsto \kappa\left(\lambda Q_{1}\right) .
\end{aligned}
$$

Moreover, its inverse $\varphi^{-1}: L \longrightarrow M$ is defined by $\varphi^{-1}(\kappa(\mu))=\pi\left(\mu T_{1}\right)$, where:

$$
U^{-1}=\left(\begin{array}{cc}
R & -\Lambda \\
T_{1} & -T_{2}
\end{array}\right) \in \mathrm{GL}_{p+q-r}(D), \quad T_{1} \in D^{(p-r) \times p}, \quad T_{2} \in D^{(p-r) \times(q-r)} .
$$

These results depend only on the residue class $\rho(\Lambda)$ of $\Lambda \in D^{q \times(q-r)}$ in the right $D$-module:

$$
\operatorname{ext}_{D}^{1}\left(M, D^{1 \times(q-r)}\right)=D^{q \times(q-r)} /\left(R D^{p \times(q-r)}\right) .
$$

Proof. Let us first check that $\varphi$ is well-defined: if $\lambda, \lambda^{\prime} \in D^{1 \times p}$ satisfy $\pi(\lambda)=\pi\left(\lambda^{\prime}\right)$, then there exists $\nu \in D^{1 \times q}$ such that $\lambda=\lambda^{\prime}+\nu R$ and using $\left(\begin{array}{ll}R & -\Lambda\end{array}\right) U=\left(\begin{array}{ll}I_{q} & 0\end{array}\right)$, where $U \in \operatorname{GL}_{p+q-r}(D)$ is defined by (21), we get $R Q_{1}=\Lambda Q_{2}$, and thus:

$$
\varphi(\pi(\lambda))=\kappa\left(\lambda Q_{1}\right)=\kappa\left(\lambda^{\prime} Q_{1}\right)+\kappa\left((\nu \Lambda) Q_{2}\right)=\kappa\left(\lambda^{\prime} Q_{1}\right)=\varphi\left(\pi\left(\lambda^{\prime}\right)\right) .
$$

Similarly, let us now prove that the following left $D$-homomorphism

$$
\begin{aligned}
& \phi: L \quad \longrightarrow M \\
& \kappa(\mu) \longmapsto \pi\left(\mu T_{1}\right),
\end{aligned}
$$

is also well-defined: if $\mu, \mu^{\prime} \in D^{1 \times(p-r)}$ satisfy $\kappa(\mu)=\kappa\left(\mu^{\prime}\right)$, then there exists $\theta \in D^{1 \times(q-r)}$ such that $\mu=\mu^{\prime}+\theta Q_{2}$ and using the identity $U U^{-1}=I_{p+q-r}$, we get $Q_{2} T_{1}=-S_{2} R$ which yields:

$$
\left.\phi(\kappa(\mu))=\pi\left(\mu T_{1}\right)\right)=\pi\left(\mu^{\prime} T_{1}\right)-\pi\left(\left(\theta S_{2}\right) R\right)=\pi\left(\mu^{\prime} T_{1}\right)=\phi\left(\kappa\left(\mu^{\prime}\right)\right) .
$$

Now, using the identity $U^{-1} U=I_{p+q-r}$, we obtain $S_{1} R+Q_{1} T_{1}=I_{p}$ and thus

$$
(\phi \circ \varphi)(\pi(\lambda))=\phi\left(\kappa\left(\lambda Q_{1}\right)\right)=\pi\left(\lambda Q_{1} T_{1}\right)=\pi(\lambda)-\pi\left(\left(\lambda S_{1}\right) R\right)=\pi(\lambda),
$$

for all $\lambda \in D^{1 \times p}$, i.e., $\phi \circ \varphi=\operatorname{id}_{M}$. Using $U U^{-1}=I_{p+q-r}$, we get $T_{1} Q_{1}-T_{2} Q_{2}=I_{p-r}$ and

$$
(\varphi \circ \phi)(\kappa(\mu))=\varphi\left(\pi\left(\mu T_{1}\right)\right)=\kappa\left(\mu T_{1} Q_{1}\right)=\kappa(\mu)+\kappa\left(\left(\mu T_{2}\right) Q_{2}\right)=\kappa(\mu),
$$


for all $\mu \in D^{1 \times(p-r)}$, i.e., $\varphi \circ \phi=\mathrm{id}_{L}$, which finally proves that $\varphi$ is an isomorphism and $\varphi^{-1}=\phi$.

Finally, the fact that the previous results depend only on the residue class $\rho(\Lambda)$ of the matrix $\Lambda \in D^{q \times(q-r)}$ in $\operatorname{ext}_{D}^{1}\left(M, D^{1 \times(q-r)}\right)=D^{q \times(q-r)} /\left(R D^{p \times(q-r)}\right)$ is a direct consequence of the equivalence of extensions defined in Section 2. This result can also be easily understood as follows: the following equivalence of identities

$$
\begin{gathered}
\left(\begin{array}{cc}
R & -\Lambda \\
T_{1} & -T_{2}
\end{array}\right)\left(\begin{array}{cc}
S_{1} & Q_{1} \\
S_{2} & Q_{2}
\end{array}\right)=I_{p+q-r} \\
\Leftrightarrow\left(\begin{array}{cc}
R & -(\Lambda+R \Theta) \\
T_{1} & -\left(T_{2}+T_{1} \Theta\right)
\end{array}\right)\left(\begin{array}{cc}
S_{1}+\Theta S_{2} & Q_{1}+\Theta Q_{2} \\
S_{2} & Q_{2}
\end{array}\right)=I_{p+q-r},
\end{gathered}
$$

for all $\Theta \in D^{q \times(q-r)}$, shows that the left $D$-homomorphisms $\varphi$ and $\varphi^{-1}=\phi$ do not depend on the choice of the representative $\Lambda$ of the equivalence class $\rho(\Lambda)$ in $\operatorname{ext}_{D}^{1}\left(M, D^{1 \times(q-r)}\right)$.

Let us illustrate Theorem 5 and Corollary 2 in a situation encounters in many explicit examples coming from applications (e.g., mathematical systems theory, control theory).

Example 9. We consider again Example 1 where we suppose that $R \in D^{q \times p}$ has full row rank and the left $D$-module $M_{1}=D^{1 \times p} /\left(D^{1 \times r} R_{1}\right)$, finitely presented by the submatrix $R_{1} \in D^{r \times p}$ of $R$, is free of rank $p-r$. If $\chi: M_{1} \longrightarrow D^{1 \times(p-r)}$ is a left $D$-isomorphism, then the extension (10) of

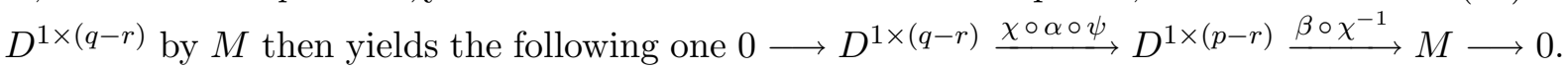
Within the standard bases of $D^{1 \times(q-r)}$ and $D^{1 \times(p-r)}$, there exists a matrix $F \in D^{(q-r) \times(p-r)}$ such that $(\chi \circ \alpha \circ \psi)(\nu)=\nu F$ for all $\nu \in D^{1 \times(q-r)}$, i.e., $M$ admits the following finite presentation:

$$
0 \longrightarrow D^{1 \times(q-r)} \stackrel{. F}{\longrightarrow} D^{1 \times(p-r)} \stackrel{\pi^{\prime}}{\longrightarrow} M \longrightarrow 0 .
$$

We find again a useful result explained in [3, 13] and implemented in the package QuILLENSusLIN ([13]) where the presentation matrix $R$ of the left $D$-module $M$ can be easily reduced. Let us give a precise description of the matrix $F$. According to 3 of Proposition 1 , there exists $U_{1} \in \mathrm{GL}_{p}(D)$ such that $R_{1} U_{1}=\left(\begin{array}{ll}I_{r} & 0\end{array}\right)$. If we write $U_{1}=\left(\begin{array}{ll}S_{1} & Q_{1}\end{array}\right)$, where $S_{1} \in D^{p \times r}$ and $Q_{1} \in D^{p \times(p-r)}$, then we have

$$
R U_{1}=\left(\begin{array}{c}
R_{1} U_{1} \\
R_{2} U_{1}
\end{array}\right)=\left(\begin{array}{cc}
I_{r} & 0 \\
R_{2} S_{1} & R_{2} Q_{1}
\end{array}\right),
$$

and the last matrix is equivalent to $\operatorname{diag}\left(I_{r}, R_{2} Q_{1}\right)$, which shows that $F=R_{2} Q_{1}$.

Let us show that this result is a also consequence of Theorem 5. If we take the matrix $\Lambda=\left(\begin{array}{ll}0^{T} & I_{q-r}^{T}\end{array}\right)^{T} \in D^{q \times(q-r)}$ and write $U^{-1}=\left(\begin{array}{ll}R_{1}^{T} & T_{1}^{T}\end{array}\right)^{T}, T_{1} \in D^{(p-r) \times p}$, then

$$
\left(\begin{array}{cc}
R_{1} & 0 \\
R_{2} & -I_{q-r} \\
T_{1} & 0
\end{array}\right)\left(\begin{array}{ccc}
S_{1} & 0 & Q_{1} \\
R_{2} S_{1} & -I_{q-r} & R_{2} Q_{1}
\end{array}\right)=I_{p+q-r}
$$

and Theorem 5 then yields $M \cong D^{1 \times(p-r)} /\left(D^{1 \times(q-r)}\left(R_{2} Q_{1}\right)\right)$.

Similarly, if $R=\left(\begin{array}{ll}R_{1} & R_{2}\end{array}\right)$, where $R_{1} \in D^{q \times r}$ and $R_{2} \in D^{q \times(p-r)}$, and if there exists $V_{1}=\left(S_{1}^{T} \quad Q_{1}^{T}\right)^{T} \in \mathrm{GL}_{q}(D)$, where $S_{1} \in D^{r \times q}$ and $Q_{1} \in D^{(q-r) \times q}$, such that

$$
V_{1} R_{1}=\left(\begin{array}{c}
S_{1} R_{1} \\
Q_{1} R_{1}
\end{array}\right)=\left(\begin{array}{c}
I_{r} \\
0
\end{array}\right),
$$


then we have

$$
V_{1} R=\left(\begin{array}{ll}
V_{1} R_{1} & V_{1} R_{2}
\end{array}\right)=\left(\begin{array}{cc}
I_{r} & S_{1} R_{2} \\
0 & Q_{1} R_{2}
\end{array}\right)
$$

and the last matrix is clearly equivalent to $\operatorname{diag}\left(I_{r}, Q_{1} R_{2}\right)$, which proves that the left $D$-module $M$ admits a finite presentation of the form (25) where $F=Q_{1} R_{2}$. Equivalently, using Example2, the extension (11) of $L=D^{1 \times(p-r)} /\left(D^{1 \times r}\left(Q_{1} R_{2}\right)\right)$ by $N_{1}=D^{1 \times r} /\left(D^{1 \times q} R_{1}\right)$ holds. Since we have $S_{1} R_{1}=I_{r}$, we get $N_{1}=0$ and thus $M \cong D^{1 \times(p-r)} /\left(D^{1 \times r}\left(Q_{1} R_{2}\right)\right)$. These two results were already obtained in [3, 13].

A straightforward consequence of Corollary 2 is the following result.

Corollary 3. Let $\mathcal{F}$ be a left $D$-module and the following two linear systems:

$$
\operatorname{ker}_{\mathcal{F}}(R .)=\left\{\eta \in \mathcal{F}^{p} \mid R \eta=0\right\}, \quad \operatorname{ker}_{\mathcal{F}}\left(Q_{2} .\right)=\left\{\zeta \in \mathcal{F}^{(p-r)} \mid Q_{2} \zeta=0\right\} .
$$

Then, we have the abelian group isomorphism $\operatorname{ker}_{\mathcal{F}}(R.) \cong \operatorname{ker}_{\mathcal{F}}\left(Q_{2}.\right)$ and:

$$
\operatorname{ker}_{\mathcal{F}}(R .)=Q_{1} \operatorname{ker}_{\mathcal{F}}\left(Q_{2} .\right), \quad \operatorname{ker}_{\mathcal{F}}\left(Q_{2} .\right)=T_{1} \operatorname{ker}_{\mathcal{F}}(R .) .
$$

Proof. Corollary 2 yields the following two commutative exact diagrams:



If $\mathcal{F}$ is a left $D$-module, then applying the contravariant left exact functor $\operatorname{hom}_{D}(\cdot, \mathcal{F})$ to the two previous commutative exact diagrams, we obtain the following ones

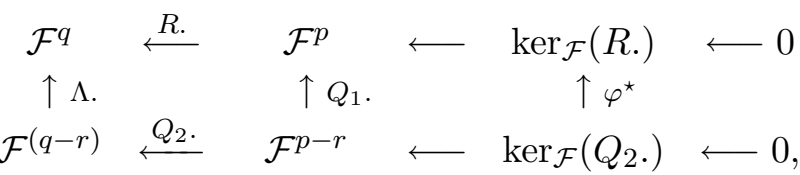



$$
\begin{aligned}
& \mathcal{F}^{(q-r)} \stackrel{Q_{2} .}{\longleftarrow} \mathcal{F}^{(p-r)} \longleftarrow \operatorname{ker}_{\mathcal{F}}\left(Q_{2} .\right) \quad \longleftarrow 0
\end{aligned}
$$

(see, e.g., [38]), which shows that $\varphi^{\star}$ is the following abelian group isomorphism:

$$
\begin{aligned}
& \varphi^{\star}: \operatorname{ker}_{\mathcal{F}}\left(Q_{2} .\right) \longrightarrow \operatorname{ker}_{\mathcal{F}}(R .) \quad\left(\varphi^{-1}\right)^{\star}: \operatorname{ker}_{\mathcal{F}}(R .) \longrightarrow \operatorname{ker}_{\mathcal{F}}\left(Q_{2} .\right) \\
& \zeta \longmapsto Q_{1} \zeta, \quad \eta \longmapsto T_{1} \eta \text {. }
\end{aligned}
$$

Corollary 4. Let $R \in D^{q \times p}$ be a full row rank matrix and $\Lambda \in D^{q \times(q-r)}$ a matrix such that $P=\left(\begin{array}{ll}R & -\Lambda\end{array}\right) \in D^{q \times(p+q-r)}$ admits a right-inverse over $D$. Then, Theorem 5 holds when $D$ satisfies one of the following properties: 
1. $D$ is a left principal ideal domain (e.g., the ring of ordinary differential operators with coefficients in a differential field such that $K=\mathbb{R}$ or $\mathbb{Q}(t)$ ),

2. $D=k\left[x_{1}, \ldots, x_{n}\right]$ is a commutative polynomial ring over a field $k$,

3. $D$ is one of the two Weyl algebras $A_{n}(k)$ or $B_{n}(k)$, where $k$ a field of characteristic 0 (e.g., $k=\mathbb{Q}, \mathbb{R}, \mathbb{C})$, and $p-r \geq 2$.

4. $D$ is the ring of ordinary differential operators with coefficients in the ring of formal power series $k \llbracket t \rrbracket$, where $k$ is a field of characteristic 0 , or in the ring of convergent power series $k\{t\}$ with coefficients in $k=\mathbb{R}$ or $\mathbb{C}$, and $p-r \geq 2$.

Proof. If $D$ satisfy one of the four conditions, then the stably free left $D$-module $E$ finitely presented by $P=\left(\begin{array}{ll}R & -\Lambda\end{array}\right) \in D^{q \times(p+q-r)}$, is free of rank $p-r$ by Theorem 3 . The result is then a consequence of Theorem 5 .

Remark 5. Corollary 4 can also be understood as follows: if the noetherian domain $D$ is a so-called Hermite ring, namely, if every finitely generated stably free left $D$-module is free, and $M=D^{1 \times p} /\left(D^{1 \times q} R\right)$ is the left $D$-module finitely presented by the full row rank matrix $R$, then $M$ can be generated by $p-r$ elements iff its Auslander transpose right $D$-module $\operatorname{ext}_{D}^{1}(M, D)=D^{q} /\left(R D^{p}\right)$ can be generated by $q-r$ elements (see Lemma 3 and Theorem 5 .

If $D$ satisfy one of the four conditions of Corollary 4 , then it is enough to search for a matrix $\Lambda \in D^{q \times(q-r)}$ such that $P=\left(\begin{array}{ll}R & -\Lambda\end{array}\right)$ admits a right-inverse over $D$ by Proposition 1 .

Let us illustrate Theorem 5 and Corollary 4 .

Example 10. We consider again Example 4 where the $D=\mathbb{Q}\left(\eta_{1}, \eta_{2}\right)\left[\partial, \sigma_{1}, \sigma_{2}\right]$-module $E=$ $D^{1 \times 7} /\left(D^{1 \times 4} P\right)$ was proved to be a stably free and thus free by Quillen-Suslin theorem (see also 2 of Corollary [4). Using constructive versions of the Quillen-Suslin theorem ([13]) and their implementation in the package QuillenSuslin ([13]) and OreModules ([6]), we obtain that

$$
U=\left(\begin{array}{ccccccc}
0 & 0 & -1 & 0 & -1 & -\sigma_{1} & 0 \\
0 & 0 & 1 & 0 & 0 & \sigma_{1} & 0 \\
0 & 0 & 0 & 1 & 0 & 0 & \sigma_{2} \\
-1 & 0 & 0 & -1 & -1 & 0 & -\sigma_{2} \\
0 & 0 & -\sigma_{1} & 0 & -\sigma_{1} & 1-\sigma_{1}^{2} & 0 \\
-\sigma_{2} & 0 & 0 & -\sigma_{2} & -\sigma_{2} & 0 & 1-\sigma_{2}^{2} \\
\eta_{2} & 1 & 2 \eta_{1} & 2 \eta_{2} & \partial+\eta_{1}+\eta_{2} & 2 \eta_{1} \sigma_{1} & 2 \eta_{2} \sigma_{2}
\end{array}\right) \in \operatorname{GL}_{7}(D)
$$

satisfies $\left(\begin{array}{ll}R & -\Lambda\end{array}\right) U=\left(\begin{array}{ll}I_{4} & 0\end{array}\right)$, and thus $Q_{2}=\left(\begin{array}{lll}\partial+\eta_{1}+\eta_{2} & 2 \eta_{1} \sigma_{1} & 2 \eta_{2} \sigma_{2}\end{array}\right)$. We then have $M=D^{1 \times 6} /\left(D^{1 \times 4} R\right) \cong L=D^{1 \times 3} /\left(D Q_{2}\right)$, i.e., using Corollary 3, 13 is equivalent to the following sole differential time-delay equation:

$$
\dot{x}_{1}(t)+\left(\eta_{1}+\eta_{2}\right) x_{1}(t)+2 \eta_{1} x_{2}\left(t-h_{1}\right)+2 \eta_{2} x_{3}\left(t-h_{2}\right)=0 .
$$

This result was already obtained in [9] after the resolutions of algebraic Riccati equations of the form $X R X=X$ (see [8, 9]). But, Serre's reduction allows us to obtain this result in a more direct way. Finally, we point out that the study of the algebraic properties of $(13)$ is now highly simplified since we can easily checked that $M \cong L$ is torsion-free and $\sigma_{1}$ and $\sigma_{2}$-free (see [25]). 
Example 11. We consider again the general transmission line (14) studied in Example 5 . If $B=K\left[\partial_{t}, \partial_{x}\right]$ is the commutative polynomial ring of differential operators in $\partial_{t}$ and $\partial_{x}$ with coefficients in the field $K=\mathbb{Q}(L, R, C, G)[\alpha] /\left(\alpha^{2}-L C\right)$ and $P=(J \quad-\Lambda) \in B^{2 \times 3}$ is the matrix formed by the matrix $J$ defined by 15$)$ and $\Lambda=\left(\begin{array}{ll}\alpha & C\end{array}\right)^{T}$, then the stably free $B$ module $E=B^{1 \times 3} /\left(B^{1 \times 2} P\right)$ is free by the Quillen-Suslin theorem. Computing a basis of $E$ using the constructive versions of the Quillen-Suslin theorem explained in [13] and implemented in QuillenSuslin ([13]) and OreModules ([6]), the matrix $U=\left(S^{T} Q^{T}\right)^{T} \in \mathrm{GL}_{3}(B)$ defined by the matrix $S \in B^{3 \times 2}$ given in Example 5 and $Q=\left(\begin{array}{ll}Q_{1}^{T} & Q_{2}^{T}\end{array}\right)^{T}$, where

$$
\left\{\begin{array}{l}
Q_{1}=\left(\alpha \partial_{x}-L C \partial_{t}-R C \quad C \partial_{x}-\alpha C \partial_{t}-\alpha G\right)^{T} \\
Q_{2}=\partial_{x}^{2}-L C \partial_{t}^{2}-(L C+R C) \partial_{t}-R G
\end{array}\right.
$$

satisfies $(J-\Lambda) U=\left(\begin{array}{ll}I_{2} & 0\end{array}\right)$. Hence, if $C \neq 0, L \neq 0$ and $L G-R C \neq 0$, then (14) is equivalent to the following sole partial differential equation:

$$
\left(\partial_{x}^{2}-L C \partial_{t}^{2}-(L C+R C) \partial_{t}-R G\right) Z(t, x)=0 .
$$

Example 12. We consider again Example 6 where we proved that the left $D=A_{2}(\mathbb{Q}(a, b))$ module $E=D^{1 \times 3} /\left(D^{1 \times 2} P\right)$ was stably free, where $P=(R-\Lambda)$ is formed by the matrix $R$ defined by $(17)$ and by $\Lambda=\left(\begin{array}{ll}a & b\end{array}\right)^{T}$. Since the rank of $E$ is $3-2=1$, we cannot use Stafford's theorem (see 3 of Theorem 3) to conclude that $E$ is a free left $D$-module of rank 1 . We need to investigate whenever or not $E$ is a free left $D$-module of rank 1 for particular values of $a$ and $b$. Using a constructive algorithm explained in [6] and implemented in OreModules ([7]), the stably free left $D$-module $E$ admits the following minimal parametrization ([6])

$$
\begin{gathered}
Q= \\
\left(\begin{array}{c}
Q= \\
-a^{2} b+b a^{2} x \partial_{x}-a^{3} x \partial_{y}-a\left(a^{2}+b^{2}\right) x^{2} \partial_{x} \partial_{y}-b\left(a^{2}+b^{2}\right) x^{2} \partial_{y}^{2} \\
a b^{2} \partial_{x}-b\left(2 b^{2}+3 a^{2}\right) \partial_{y}-b\left(a^{2}+b^{2}\right) x \partial_{x} \partial_{y}+a\left(a^{2}+b^{2}\right) x \partial_{y}^{2} \\
-a^{2} \partial_{y}-\left(a^{2}+b^{2}\right) x^{2} \partial_{y} \partial_{x}^{2}+a b x \partial_{x}^{2}-3\left(a^{2}+b^{2}\right) x \partial_{x} \partial_{y}+a b x \partial_{y}^{2}-\left(a^{2}+b^{2}\right) x^{2} \partial_{y}^{3}
\end{array}\right),
\end{gathered}
$$

namely, $E \cong D^{1 \times 3} Q=\sum_{i=1}^{3} D Q_{i 1}$, i.e., $E$ is isomorphic to the left ideal of $D$ generated by the three entries of $Q$. Therefore, the following long exact sequence

$$
0 \longrightarrow D^{1 \times 2} \stackrel{. P}{\longrightarrow} D^{1 \times 3} \stackrel{\cdot Q}{\longrightarrow} D \stackrel{\sigma}{\longrightarrow} N \longrightarrow 0,
$$

holds, where $\sigma: D \longrightarrow N$ is the canonical projection onto $N=D /\left(D^{1 \times 3} Q\right)$. If there exists a set of values for the arbitrary constant parameters $a$ and $b$ such that the left $D$-module $N$ vanishes, then the last long exact sequence shows that $D^{1 \times 3} Q=D$, and thus $E \cong D^{1 \times 3} Q=D$ is a free left $D$-module of rank 1 . Computing a Gröbner basis of the left $D$-module $D^{1 \times 3} Q$, we obtain that the generator $z=\sigma(1)$ of $N$ satisfies the equation $d z=0$, where:

$$
d=-\left(a^{2}+b^{2}\right)^{2} x^{2} \partial_{y}^{2}+2 a b\left(a^{2}+b^{2}\right) x \partial_{y}-a^{2} b^{2} \in D .
$$

Therefore, if we consider a solution of the following polynomial system

$$
\left\{\begin{array} { l } 
{ ( a ^ { 2 } + b ^ { 2 } ) ^ { 2 } = 0 , } \\
{ a b ( a ^ { 2 } + b ^ { 2 } ) = 0 , } \\
{ a ^ { 2 } b ^ { 2 } = - 1 , }
\end{array} \Leftrightarrow \left\{\begin{array} { l } 
{ a ^ { 2 } + b ^ { 2 } = 0 , } \\
{ a ^ { 2 } b ^ { 2 } = - 1 , }
\end{array} \Leftrightarrow \left\{\begin{array} { l } 
{ b ^ { 2 } = - a ^ { 2 } , } \\
{ a ^ { 4 } = 1 , }
\end{array} \Leftrightarrow \left\{\begin{array}{l}
b= \pm i a, \\
a \in\{ \pm 1, \pm i\}
\end{array}\right.\right.\right.\right.
$$


such as, e.g., $a=1$ and $b=i$, then $d$ is then reduced to 1 . If we consider the new ring

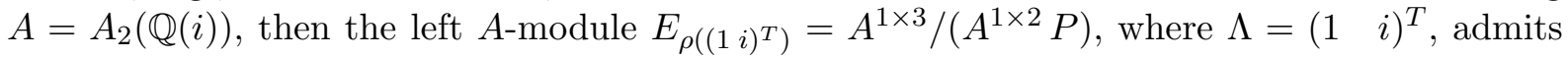
the following injective parametrization

$$
Q=\left(\begin{array}{c}
x\left(i \partial_{x}-\partial_{y}\right)-i \\
-\left(\partial_{x}+i \partial_{y}\right) \\
i x\left(\partial_{x}^{2}+\partial_{y}^{2}\right)-\partial_{y}
\end{array}\right)
$$

and $T=\left(\begin{array}{lll}i & -x & 0\end{array}\right)$ is a left-inverse of $Q$ over $A$. Using Theorem 5 and Corollary 3 , we finally obtain $M \cong A /\left(A\left(i x\left(\partial_{x}^{2}+\partial_{y}^{2}\right)-\partial_{y}\right)\right)$ and:

$$
\left.6 \quad \Leftrightarrow \quad\left(i x\left(\partial_{x}^{2}+\partial_{y}^{2}\right)-\partial_{y}\right)\right) u=0 \quad \Leftrightarrow \quad\left(x\left(\partial_{x}^{2}+\partial_{y}^{2}\right)+i \partial_{y}\right) u=0 .
$$

Let us now study when the matrix $R$ is equivalent to $\operatorname{diag}\left(I_{r}, Q_{2}\right)$, where $\operatorname{diag}\left(I_{r}, Q_{2}\right)$ denotes the block-diagonal matrix formed by the blocks $I_{r}$ and $Q_{2}$.

Corollary 5. With the notations of Theorem 5 and Corollary 2, if the matrix $\Lambda \in D^{q \times(q-r)}$ admits a left-inverse $\Gamma \in D^{(q-r) \times q}$, i.e., $\Gamma \Lambda=I_{q-r}$, then the matrix $Q_{1}$ admits the left-inverse $T_{1}-T_{2} \Gamma R \in D^{(p-r) \times p}$ and the left $D$-module $\operatorname{ker}_{D}\left(. Q_{1}\right)$ is stably free of rank $r$.

Moreover, if the left $D$-module $\operatorname{ker}_{D}\left(. Q_{1}\right)$ is free of rank $r$, then there exists $Q_{3} \in D^{p \times r}$ such that $W \triangleq\left(\begin{array}{ll}Q_{3} & Q_{1}\end{array}\right) \in \mathrm{GL}_{p}(D)$. If we write $W^{-1}=\left(\begin{array}{ll}Y_{3}^{T} & Y_{1}^{T}\end{array}\right)^{T}$, where $Y_{3} \in D^{r \times p}$ and $Y_{1} \in D^{(p-r) \times p}$, then the matrix $X \triangleq\left(\begin{array}{ll}R Q_{3} & \Lambda\end{array}\right)$ is unimodular, i.e., $X \in \mathrm{GL}_{q}(D)$ and:

$$
V \triangleq X^{-1}=\left(\begin{array}{c}
Y_{3} S_{1} \\
Q_{2} Y_{1} S_{1}-S_{2}
\end{array}\right) .
$$

The matrix $R$ is then equivalent to the matrix $X \operatorname{diag}\left(I_{r}, Q_{2}\right) W^{-1}$ or equivalently:

$$
V R W=\left(\begin{array}{cc}
I_{r} & 0 \\
0 & Q_{2}
\end{array}\right) .
$$

Finally, the left D-module $\operatorname{ker}_{D}\left(. Q_{1}\right)$ is free when D satisfies 1 or 2 of Corollary 4 or if $D$ is $A_{n}(k)$ or $B_{n}(k)$ over a field $k$ of characteristic 0 and $r \geq 2$ or if $D$ is the ring of ordinary differential operators with coefficients in $k \llbracket t \rrbracket$, where $k$ a field of characteristic 0 , or with coefficients in $k\{t\}$, where $k=\mathbb{R}$ or $\mathbb{C}$, and $r \geq 2$.

Proof. By hypothesis, the following Bézout identity holds:

$$
\left(\begin{array}{cc}
R & -\Lambda \\
T_{1} & -T_{2}
\end{array}\right)\left(\begin{array}{ll}
S_{1} & Q_{1} \\
S_{2} & Q_{2}
\end{array}\right)=I_{p+q-r} \Leftrightarrow\left\{\begin{array}{l}
R S_{1}-\Lambda S_{2}=I_{q} \\
R Q_{1}=\Lambda Q_{2} \\
T_{1} S_{1}=T_{2} S_{2} \\
T_{1} Q_{1}-T_{2} Q_{2}=I_{p-r}
\end{array}\right.
$$

Moreover, we know that there exists $\Gamma \in D^{(q-r) \times q}$ such that $\Gamma \Lambda=I_{q-r}$. Pre-multiplying the second equation of $(29)$ by $\Gamma$, we get $Q_{2}=\Gamma R Q_{1}$, which, combined with the last equation of (29), yields $\left(T_{1}-T_{2} \Gamma R\right) Q_{1}=I_{p-r}$ and proves that $Q_{1}$ admits a left-inverse over $D$. Therefore, the following short exact sequence

$$
0 \longrightarrow \operatorname{ker}_{D}\left(. Q_{1}\right) \stackrel{i}{\longrightarrow} D^{1 \times p} \stackrel{\cdot Q_{1}}{\longrightarrow} D^{1 \times(p-r)} \longrightarrow 0
$$


splits, namely, we have $D^{1 \times p} \cong \operatorname{ker}_{D}\left(. Q_{1}\right) \oplus D^{1 \times(p-r)}$ (see, e.g., [38]), which proves that $\operatorname{ker}_{D}\left(. Q_{1}\right)$ is a stably free left $D$-module of rank $p-(p-r)=r$.

Now, let us suppose that $\operatorname{ker}_{D}\left(. Q_{1}\right)$ is a free left $D$-module of rank $r$ and let denote by $\psi: D^{1 \times r} \longrightarrow \operatorname{ker}_{D}\left(. Q_{1}\right)$ a left $D$-isomorphism. The split short exact sequence 30 then yields

$$
0 \longrightarrow D^{1 \times r} \underset{\stackrel{Q_{3}}{\rightleftarrows}}{\stackrel{. Y_{3}}{\longrightarrow}} D^{1 \times p} \underset{\stackrel{. Y_{1}}{\stackrel{. Q_{1}}{\longrightarrow}}}{\stackrel{.}{\leftrightarrows}} D^{1 \times(p-r)} \longrightarrow 0,
$$

where $Y_{3} \in D^{r \times p}$ is a matrix such that $(i \circ \psi)(\nu)=\nu Y_{3}$ for all $\nu \in D^{1 \times r}$. Hence, if we write $W=\left(\begin{array}{ll}Q_{3} & Q_{1}\end{array}\right) \in D^{p \times p}$, then the previous split short exact sequence yields

$$
\left(\begin{array}{ll}
Q_{3} & Q_{1}
\end{array}\right)\left(\begin{array}{c}
Y_{3} \\
Y_{1}
\end{array}\right)=Q_{3} Y_{3}+Q_{1} Y_{1}=I_{p}, \quad\left(\begin{array}{c}
Y_{3} \\
Y_{1}
\end{array}\right)\left(\begin{array}{ll}
Q_{3} & Q_{1}
\end{array}\right)=\left(\begin{array}{cc}
I_{r} & 0 \\
0 & I_{p-r}
\end{array}\right)=I_{p},
$$

i.e., $W \in \mathrm{GL}_{p}(D)$ and $W^{-1}=\left(\begin{array}{ll}Y_{3}^{T} & Y_{1}^{T}\end{array}\right)^{T}$. The second identity of 29 yields:

$$
R W=R\left(\begin{array}{ll}
Q_{3} & Q_{1}
\end{array}\right)=\left(\begin{array}{ll}
R Q_{3} & R Q_{1}
\end{array}\right)=\left(\begin{array}{ll}
R Q_{3} & \Lambda Q_{2}
\end{array}\right)=\left(\begin{array}{ll}
R Q_{3} & \Lambda
\end{array}\right)\left(\begin{array}{cc}
I_{r} & 0 \\
0 & Q_{2}
\end{array}\right) .
$$

The first identity of (32) and the first and the second identities of $(29)$ imply

$$
\begin{aligned}
& \left(\begin{array}{ll}
R Q_{3} & \Lambda
\end{array}\right)\left(\begin{array}{c}
Y_{3} S_{1} \\
Q_{2} Y_{1} S_{1}-S_{2}
\end{array}\right)=R Q_{3} Y_{3} S_{1}+\Lambda Q_{2} Y_{1} S_{1}-\Lambda S_{2} \\
& =R S_{1}-R Q_{1} Y_{1} S_{1}+\Lambda Q_{2} Y_{1} S_{1}-\Lambda S_{2} \\
& =I_{q}-\left(R Q_{1}-\Lambda Q_{2}\right) Y_{1} S_{1}=I_{q} \text {, }
\end{aligned}
$$

which implies $X \triangleq\left(\begin{array}{ll}R Q_{3} & \Lambda\end{array}\right) \in \mathrm{GL}_{q}(D)$ since $D$ is a noetherian ring and thus a stably finite ring (i.e., a ring over which every left or right invertible matrix is invertible ([19])) and:

$$
V \triangleq X^{-1}=\left(\begin{array}{c}
Y_{3} S_{1} \\
Q_{2} Y_{1} S_{1}-S_{2}
\end{array}\right)
$$

Using (33), we finally obtain $V R W=\operatorname{diag}\left(I_{r}, Q_{2}\right)$, which proves the corollary.

Let us illustrate Corollary 5 with explicit examples.

Example 13. We consider again Examples 4 and 10 . Since $\Lambda$ clearly admits a left-inverse over $D$, we can check that the matrix $Q_{1} \in D^{6 \times 3}$ defined by the first 6 rows of $Q$ also admits a right-inverse over $D$. Using constructive versions of the Quillen-Suslin theorem and their implementations in QUILLENSUSLIN and OREModules, we can complete the matrix $Q_{1}$ to the following unimodular matrix:

$$
W=\left(\begin{array}{ll}
Q_{3} & Q_{1}
\end{array}\right)=\left(\begin{array}{cccccc}
1 & 0 & 0 & -1 & -\sigma_{1} & 0 \\
0 & -1 & 0 & 0 & \sigma_{1} & 0 \\
0 & 0 & 1 & 0 & 0 & \sigma_{2} \\
0 & -1 & -1 & -1 & 0 & -\sigma_{2} \\
0 & 0 & 0 & -\sigma_{1} & 1-\sigma_{1}^{2} & 0 \\
0 & -\sigma_{2} & -\sigma_{2} & -\sigma_{2} & 0 & 1-\sigma_{2}^{2}
\end{array}\right)^{T} \in \operatorname{GL}_{6}(D)
$$


We can now check that the following matrix

$$
X=\left(\begin{array}{ll}
R Q_{3} & \Lambda
\end{array}\right)=\left(\begin{array}{cccc}
1 & 0 & 0 & 0 \\
\partial+\eta_{1} & -\partial+\eta_{1}-\eta_{2} & -2 \eta_{2} & -1 \\
\sigma_{1}^{2} & -1 & 0 & 0 \\
0 & 0 & 1 & 0
\end{array}\right) \in D^{4 \times 4}
$$

is unimodular over $D$, i.e., $X \in \mathrm{GL}_{4}(D)$, and satisfies

$$
R W=X \operatorname{diag}\left(I_{3}, Q_{2}\right) \quad \Leftrightarrow \quad \operatorname{diag}\left(I_{3}, Q_{2}\right)=X^{-1} R W,
$$

which finally proves that $R$ is equivalent to $\operatorname{diag}\left(I_{3}, Q_{2}\right)$.

Example 14. We consider again Examples 5 and 11. We can easily check that $\Lambda$ admits a left-inverse over $B$. Using Corollary 5 , the matrix $Q_{1} \in B^{2}$ defined by the first 2 entries of $Q$ admits a right-inverse over $B$. Then, using constructive versions of the Quillen-Suslin theorem and their implementations in QuillenSusLin and OreModules, we can complete $Q_{1}$ to the following unimodular matrix:

$$
W=\left(\begin{array}{ll}
Q_{3} & Q_{1}
\end{array}\right)=\left(\begin{array}{cc}
\frac{\alpha}{C(R C-L G)} & -C\left(L \partial_{t}+R\right)+\alpha \partial_{x} \\
\frac{1}{R C-L G} & C\left(\partial_{x}-\alpha \partial_{t}\right)-\alpha G
\end{array}\right) \in \mathrm{GL}_{2}(A) .
$$

Moreover, we can check that the following matrix

$$
X=\left(\begin{array}{ll}
J Q_{3} & \Lambda
\end{array}\right)=\left(\begin{array}{cc}
\frac{\alpha \partial_{x}+C\left(L \partial_{t}+R\right)}{C(R C-L G)} & \alpha \\
\frac{C\left(\partial_{x}+\alpha \partial_{t}\right)+\alpha G}{C(R C-L G)} & C
\end{array}\right) \in B^{2 \times 2}
$$

is unimodular over $B$, i.e., $X \in \mathrm{GL}_{2}(B)$, and satisfies

$$
J W=X \operatorname{diag}\left(1, Q_{2}\right) \quad \Leftrightarrow \quad X^{-1} J W=\operatorname{diag}\left(1, Q_{2}\right),
$$

which proves that the matrix $R$ is equivalent to $\operatorname{diag}\left(1, Q_{2}\right)$.

Example 15. We consider again Examples 6 and 12 . Since $\Lambda=\left(\begin{array}{ll}1 & i\end{array}\right)^{T}$ admits the leftinverse $\Gamma=\left(\begin{array}{ll}1 & 0\end{array}\right)$ over $A$, Corollary 5 shows that the matrix $R$ defined by (17) is equivalent to $\left.\operatorname{diag}\left(1, i x\left(\partial_{x}^{2}+\partial_{y}^{2}\right)-\partial_{y}\right)\right)$. If $Q_{1}$ denotes the column vector formed by the first two entries of (28), then $\operatorname{ker}_{A}\left(. Q_{1}\right)=A\left(-i \partial_{x}+\partial_{y} \quad x\left(\partial_{x}+i \partial_{y}\right)\right) \cong A$, i.e., $\operatorname{ker}_{A}\left(. Q_{1}\right)$ is a free left $A$-module of rank 1. Since $Q_{3}=\left(\begin{array}{ll}i x & -1\end{array}\right)^{T}$ is a right-inverse of $\left(-i \partial_{x}+\partial_{y} \quad x\left(\partial_{x}+i \partial_{y}\right)\right)$ over $A$, we obtain the unimodular matrix:

$$
W=\left(\begin{array}{cc}
i x & x\left(i \partial_{x}-\partial_{y}\right)-i \\
-1 & -\partial_{x}-i \partial_{y}
\end{array}\right), \quad W^{-1}=\left(\begin{array}{cc}
-i \partial_{x}+\partial_{y} & x\left(\partial_{x}+i \partial_{y}\right) \\
i & -x
\end{array}\right)
$$

Moreover, using Corollary 5, we can also introduce the unimodular matrices:

$$
\begin{aligned}
& X=\left(\begin{array}{ll}
R Q_{3} & \Lambda
\end{array}\right)=\left(\begin{array}{cc}
x\left(i \partial_{x}+\partial_{y}\right)+i & 1 \\
-x\left(\partial_{x}-i \partial_{y}\right) & i
\end{array}\right) \\
& V=X^{-1}=\left(\begin{array}{cc}
-i & 1 \\
-x\left(\partial_{x}-i \partial_{y}\right) & -x\left(i \partial_{x}+\partial_{y}\right)-i
\end{array}\right) .
\end{aligned}
$$

Finally, we can easily check that $\left.V R W=\operatorname{diag}\left(1, i x\left(\partial_{x}^{2}+\partial_{y}^{2}\right)-\partial_{y}\right)\right)$. 
Example 16. Let us consider a model of a two reflector antenna studied in [18, 26] which is defined by the linear differential time-delay $\operatorname{system} \operatorname{ker}_{\mathcal{F}}(R$.), where

$$
R=\left(\begin{array}{ccccccccc}
\partial & -K_{1} & 0 & 0 & 0 & 0 & 0 & 0 & 0 \\
0 & \partial+\frac{K_{2}}{T_{e}} & 0 & 0 & 0 & 0 & -\frac{K_{p}}{T_{e}} \delta & -\frac{K_{c}}{T_{e}} \delta & -\frac{K_{c}}{T_{e}} \delta \\
0 & 0 & \partial & -K_{1} & 0 & 0 & 0 & 0 & 0 \\
0 & 0 & 0 & \partial+\frac{K_{2}}{T_{e}} & 0 & 0 & -\frac{K_{c}}{T_{e}} \delta & -\frac{K_{p}}{T_{e}} \delta & -\frac{K_{c}}{T_{e}} \delta \\
0 & 0 & 0 & 0 & \partial & -K_{1} & 0 & 0 & 0 \\
0 & 0 & 0 & 0 & 0 & \partial+\frac{K_{2}}{T_{e}} & -\frac{K_{c}}{T_{e}} \delta & -\frac{K_{c}}{T_{e}} \delta & -\frac{K_{p}}{T_{e}} \delta
\end{array}\right),
$$

$\partial y(t)=\dot{y}(t), \delta y(t)=y(t-1)$ for all $y \in \mathcal{F}=C^{\infty}(\mathbb{R})$, and $K_{1}, K_{2}, K_{c}, K_{e}, K_{p}$ and $T_{e}$ are constant parameters. Let $D=\mathbb{Q}\left(K_{1}, K_{2}, K_{c}, K_{e}, T_{e}\right)[\partial, \delta]$ be the commutative polynomial ring of differential time-delay operators and $M=D^{1 \times 9} /\left(D^{1 \times 6} R\right)$ the $D$-module finitely presented by $R$. If we introduce the following matrix

$$
\Lambda=\left(\begin{array}{ccc}
0 & 0 & 0 \\
1 & 0 & 0 \\
0 & 0 & 0 \\
0 & 1 & 0 \\
0 & 0 & 0 \\
0 & 0 & 1
\end{array}\right) \in D^{6 \times 3},
$$

then the matrix $S \in D^{12 \times 6}$ defined in Figure 1 is a right-inverse of $P=(R-\Lambda) \in D^{6 \times 12}$. Hence, the $D$-module $E=D^{1 \times 12} /\left(D^{1 \times 6} P\right)$ is projective, and thus free by the Quillen-Suslin theorem. Using the packages QuiLlenSUSLIN or OREModules, we can compute a basis and an injective parametrization of $E$. We get that the matrix $Q \in D^{12 \times 6}$ given in Figure 1 defines an injective parametrization of $E$, i.e., $\operatorname{ker}_{D}(. Q)=D^{1 \times 6} P \cong D^{1 \times 6}$. Using Theorem 5 and Corollary 3 , we obtain that $M \cong L=D^{1 \times 6} /\left(D^{1 \times 3} Q_{2}\right)$, where $Q_{2}$ is the matrix defined by the last three rows of $Q$, and thus $\operatorname{ker}_{\mathcal{F}}(R$. $) \cong \operatorname{ker}_{\mathcal{F}}\left(Q_{2}\right.$. $)$, i.e.:

$$
\left\{\begin{array}{l}
T_{e} \ddot{\zeta}_{1}(t)+K_{2} \dot{\zeta}_{1}(t)+\left(K_{p}+2 K_{c}\right)\left(K_{c}-K_{p}\right) \zeta_{2}(t-1)=0, \\
T_{e} \ddot{\zeta}_{3}(t)+K_{2} \dot{\zeta}_{3}(t)+\left(K_{p}+2 K_{c}\right)\left(K_{c}-K_{p}\right) \zeta_{4}(t-1)=0, \\
T_{e} \ddot{\zeta}_{5}(t)+K_{2} \dot{\zeta}_{5}(t)+\left(K_{p}+2 K_{c}\right)\left(K_{c}-K_{p}\right) \zeta_{6}(t-1)=0 .
\end{array}\right.
$$

We note the equations of the previous system are uncoupled, i.e.:

$$
M \cong\left[D^{1 \times 2} /\left(D\left(\left(T_{e} \partial+K_{2}\right) \partial \quad\left(K_{p}+2 K_{c}\right)\left(K_{c}-K_{p}\right) \delta\right)\right]^{3} .\right.
$$

We note that $\Lambda$ admits a left-inverse $\Gamma$ over $D$ defined by:

$$
\Gamma=\left(\begin{array}{llllll}
0 & 1 & 0 & 0 & 0 & 0 \\
0 & 0 & 0 & 1 & 0 & 0 \\
0 & 0 & 0 & 0 & 0 & 1
\end{array}\right)
$$

Hence, let us compute $V \in \mathrm{GL}_{6}(D)$ and $W \in \mathrm{GL}_{9}(D)$ such that $V R W=\operatorname{diag}\left(I_{3}, Q_{2}\right)$. The $D$-module $\operatorname{ker}_{D}\left(. Q_{1}\right)$ is a stably free and thus a free $D$-module of rank 3 by the Quillen-Suslin 


$$
\begin{aligned}
& S=\left(\begin{array}{cccccc}
0 & 0 & 0 & 0 & 0 & 0 \\
-\frac{1}{K_{1}} & 0 & 0 & 0 & 0 & 0 \\
0 & 0 & 0 & 0 & 0 & 0 \\
0 & 0 & -\frac{1}{K_{1}} & 0 & 0 & 0 \\
0 & 0 & 0 & 0 & 0 & 0 \\
0 & 0 & 0 & 0 & -\frac{1}{K_{1}} & 0 \\
0 & 0 & 0 & 0 & 0 & 0 \\
0 & 0 & 0 & 0 & 0 & 0 \\
0 & 0 & 0 & 0 & 0 & 0 \\
-\frac{T_{e}+K_{2}}{K_{1} T_{e}} \partial & -1 & 0 & 0 & 0 & 0 \\
0 & 0 & -\frac{T_{e}+K_{2}}{K_{1} T_{e}} \partial & -1 & 0 & 0 \\
0 & 0 & 0 & 0 & -\frac{T_{e}+K_{2}}{K_{1} T_{e}} \partial & -1
\end{array}\right) \\
& Q=\left(\begin{array}{ccc}
K_{1} T_{e} & 0 & 0 \\
T_{e} \partial & 0 & 0 \\
0 & 0 & K_{1} T_{e} \\
0 & 0 & T_{e} \partial \\
0 & 0 & 0 \\
0 & T_{e}\left(K_{p}+K_{c}\right) & 0 \\
0 & -K_{c} T_{e} & 0 \\
0 & -K_{c} T_{e} & 0 \\
0 & 0 & 0 \\
\left(T_{e} \partial+K_{2}\right) \partial & \left(K_{p}+2 K_{c}\right)\left(K_{c}-K_{p}\right) \delta & \left(T_{e} \partial+K_{2}\right) \partial \\
0 & 0 & 0 \\
0 & &
\end{array}\right. \\
& \left.\begin{array}{ccc}
0 & 0 & 0 \\
0 & 0 & 0 \\
0 & 0 & 0 \\
0 & 0 & 0 \\
0 & K_{1} T_{e} & 0 \\
0 & T_{e} \partial & 0 \\
-K_{c} T_{e} & 0 & -K_{c} T_{e} \\
T_{e}\left(K_{p}+K_{c}\right) & 0 & -K_{c} T_{e} \\
-K_{c} T_{e} & 0 & T_{e}\left(K_{p}+K_{c}\right) \\
0 & 0 & 0 \\
\left(K_{p}+2 K_{c}\right)\left(K_{c}-K_{p}\right) \delta & 0 & 0 \\
0 & \left(T_{e} \partial+K_{2}\right) \partial & \left(2 K_{c}+K_{p}\right)\left(K_{c}-K_{p}\right) \delta
\end{array}\right)
\end{aligned}
$$

Figure 1: Matrices $S$ and $Q$ 
theorem. This last result can be checked again by computing $\operatorname{ker}_{D}\left(. Q_{1}\right)$ : we have $\operatorname{ker}_{D}\left(. Q_{1}\right)=$ $D^{1 \times 3} F \cong D^{1 \times 3}$, where the full row rank matrix $F \in D^{3 \times 9}$ is defined by:

$$
F=\left(\begin{array}{ccccccccc}
\partial & -K_{1} & 0 & 0 & 0 & 0 & 0 & 0 & 0 \\
0 & 0 & \partial & -K_{1} & 0 & 0 & 0 & 0 & 0 \\
0 & 0 & 0 & 0 & \partial & -K_{1} & 0 & 0 & 0
\end{array}\right)
$$

Computing a right-inverse of $F$, we obtain that the matrix $Q_{3} \in D^{9 \times 3}$ defined by

$$
Q_{3}=-\frac{1}{K_{1}}\left(\begin{array}{ccc}
0 & 0 & 0 \\
1 & 0 & 0 \\
0 & 0 & 0 \\
0 & 1 & 0 \\
0 & 0 & 0 \\
0 & 0 & 1 \\
0 & 0 & 0 \\
0 & 0 & 0 \\
0 & 0 & 0
\end{array}\right)
$$

is such that the matrix $W$ defined by

$$
\begin{aligned}
& W=\left(\begin{array}{ll}
Q_{3} & Q_{1}
\end{array}\right)= \\
& \left(\begin{array}{cccccc}
0 & 0 & 0 & K_{1} T_{e} & 0 & 0 \\
-K_{1}^{-1} & 0 & 0 & T_{e} \partial & 0 & 0 \\
0 & 0 & 0 & 0 & 0 & K_{1} T_{e} \\
0 & -K_{1}^{-1} & 0 & 0 & 0 & T_{e} \partial \\
0 & 0 & 0 & 0 & 0 & 0 \\
0 & 0 & -K_{1}^{-1} & 0 & 0 & 0 \\
0 & 0 & 0 & 0 & T_{e}\left(K_{p}+K_{c}\right) & 0 \\
0 & 0 & 0 & 0 & -K_{c} T_{e} & 0 \\
0 & 0 & 0 & 0 & -K_{c} T_{e} & 0
\end{array}\right. \\
& \left.\begin{array}{ccc}
0 & 0 & 0 \\
0 & 0 & 0 \\
0 & 0 & 0 \\
0 & 0 & 0 \\
0 & K_{1} T_{e} & 0 \\
0 & T_{e} \partial & 0 \\
-K_{c} T_{e} & 0 & -K_{c} T_{e} \\
T_{e}\left(K_{p}+K_{c}\right) & 0 & -K_{c} T_{e} \\
-K_{c} T_{e} & 0 & T_{e}\left(K_{p}+K_{c}\right)
\end{array}\right)
\end{aligned}
$$


is unimodular, i.e., $W \in \mathrm{GL}_{9}(D)$. Forming the matrix $X=\left(\begin{array}{ll}R Q_{3} \quad \Lambda & \Lambda\end{array} D^{6 \times 6}\right.$, namely,

$$
X=\left(\begin{array}{cccccc}
1 & 0 & 0 & 0 & 0 & 0 \\
-\frac{T_{e} \partial+K_{2}}{K_{1} T_{e}} & 0 & 0 & 1 & 0 & 0 \\
0 & 1 & 0 & 0 & 0 & 0 \\
0 & -\frac{T_{e} \partial+K_{2}}{K_{1} T_{e}} & 0 & 0 & 1 & 0 \\
0 & 0 & 1 & 0 & 0 & 0 \\
0 & 0 & -\frac{T_{e} \partial+K_{2}}{K_{1} T_{e}} & 0 & 0 & 1
\end{array}\right),
$$

then $X \in \mathrm{GL}_{6}(D)$. Its inverse is defined by

$$
V=X^{-1}=\left(\begin{array}{cccccc}
1 & 0 & 0 & 0 & 0 & 0 \\
0 & 0 & 1 & 0 & 0 & 0 \\
0 & 0 & 0 & 0 & 1 & 0 \\
\frac{T_{e} \partial+K_{2}}{K_{1} T_{e}} & 1 & 0 & 0 & 0 & 0 \\
0 & 0 & \frac{T_{e} \partial+K_{2}}{K_{1} T_{e}} & 1 & 0 & 0 \\
0 & 0 & 0 & 0 & \frac{T_{e} \partial+K_{2}}{K_{1} T_{e}} & 1
\end{array}\right)
$$

and $\bar{R}=V R W$ has finally the form $\operatorname{diag}\left(I_{3}, Q_{2}\right)$ :

$$
\begin{aligned}
& \bar{R}=V R W=\left(\begin{array}{cccccc}
1 & 0 & 0 & 0 & 0 & 0 \\
0 & 1 & 0 & 0 & 0 & 0 \\
0 & 0 & 1 & 0 & 0 & 0 \\
0 & 0 & 0 & \left(T_{e} \partial+K_{2}\right) \partial & \left(K_{p}+2 K_{c}\right)\left(K_{c}-K_{p}\right) \delta & 0 \\
0 & 0 & 0 & 0 & 0 & 0 \\
0 & 0 & 0 & 0 & 0 &
\end{array}\right. \\
& \left.\begin{array}{lccc}
0 & 0 & 0 & 0 \\
0 & 0 & 0 & 0 \\
0 & 0 & 0 & 0 \\
0 & 0 & 0 & 0 \\
\left.+K_{2}\right) \partial & \left(K_{p}+2 K_{c}\right)\left(K_{c}-K_{p}\right) \delta & 0 & 0 \\
0 & 0 & \left(T_{e} \partial+K_{2}\right) \partial & \left(K_{p}+2 K_{c}\right)\left(K_{c}-K_{p}\right) \delta
\end{array}\right) .
\end{aligned}
$$

Finally, the $D$-module $L=D^{1 \times 2} /\left(D\left(\left(T_{e} \partial+K_{2}\right) \partial \quad\left(K_{p}+2 K_{c}\right)\left(K_{c}-K_{p}\right) \delta\right)\right.$ is clearly torsionfree and $\delta$-free ([26]) and, using (34), and thus so is $M \cong N^{3}$ (see also [26]).

The next example shows that the previous results can also be used to compute the minimal number of generators of certain finitely presented modules. 
Example 17. Let us consider the commutative polynomial ring $D=\mathbb{Q}\left[\partial_{x}, \partial_{y}\right]$ and the $D$ module $M=D^{1 \times 3} /\left(D^{1 \times 2} R\right)$ finitely presented by $R$ defined by:

$$
R=\left(\begin{array}{ccc}
\partial_{x} & \partial_{y} & 0 \\
0 & \partial_{x} & \partial_{y}
\end{array}\right) \in D^{2 \times 3}
$$

The matrix $R$ defines the equation of equilibrium of the stress tensor in $\mathbb{R}^{2}$, namely:

$$
\left\{\begin{array}{l}
\partial_{x} \sigma^{11}+\partial_{y} \sigma^{12}=0 \\
\partial_{x} \sigma^{12}+\partial_{y} \sigma^{22}=0
\end{array}\right.
$$

We can easily check that the $D$-module $\operatorname{ext}_{D}^{1}(M, D) \cong D^{1 \times 2} /\left(D^{1 \times 3} R^{T}\right)$ is a $\mathbb{Q}$-vector space of dimension 3 and a basis of $\operatorname{ext}_{D}^{1}(M, D)$ is defined by the vectors $\tau\left(\left(\begin{array}{ll}1 & 0\end{array}\right)^{T}\right), \tau\left(\left(\begin{array}{ll}0 & 1\end{array}\right)^{T}\right)$ and $\tau\left(\left(\begin{array}{ll}0 & \partial_{x}\end{array}\right)^{T}\right)$, where $\tau: D^{2} \longrightarrow \operatorname{ext}_{D}^{1}(M, N)=D^{2} /\left(R D^{3}\right)$ is the canonical projection. Hence, without loss of generality, we can assume that $\Lambda$ has the form of $\Lambda=\left(a b+c \partial_{x}\right)^{T}$, where $a, b$ and $c$ are three arbitrary constants. Considering the ring $A=\mathbb{Q}[a, b, c]\left[\partial_{x}, \partial_{y}\right]$,


$\operatorname{ext}_{A}^{1}(E, A) \cong N=A^{1 \times 2} /\left(A^{1 \times 4} P^{T}\right)$ and using the algorithms developed in [6] and implemented in OreModules, we can check that $t(E) \cong \operatorname{ext}_{A}^{1}(N, A)=0$ and $\operatorname{ext}_{A}^{2}(N, A)=A /\left(\partial_{x}, \partial_{y}\right) \neq 0$. Hence, using ([6]), we obtain that the $A$-module $E$ is a torsion-free but not projective whatever the values of the parameters $a, b$ and $c$ are, which finally proves that (36) cannot be defined by sole partial differential equation with constant coefficients and the minimal number of generators $\mu(M)$ of the $D$-module $M$ is 3 .

A similar comment holds for Cosserat's system also studied in linear elasticity:

$$
\left\{\begin{array}{l}
\partial_{x} \sigma^{11}+\partial_{y} \sigma^{12}=0, \\
\partial_{x} \mu^{1}+\partial_{y} \mu^{2}+\sigma^{21}-\sigma^{12}=0, \\
\partial_{x} \sigma^{21}+\partial_{y} \sigma^{22}=0 .
\end{array}\right.
$$

Example 18. Using Example 8 and 2 of Corollary 4 , the matrix $R$ defined by 20 is not equivalent to its Smith normal form $\operatorname{diag}\left(1, x^{2}-y^{2}(y+1)\right)$. This result was first obtained in [20] (see also [3]). However, the stably free $D=k[x, y]$-module $E^{\prime}$ of rank 1 defined in Example 8 is free by the Quillen-Suslin theorem. Therefore, $R^{\prime}$ is equivalent to its Smith normal defined by $\operatorname{diag}\left(1, x^{2}-y^{2}(y+1)\right)([20])$ and we have $V R W=\operatorname{diag}\left(1, x^{2}-y^{2}(y+1)\right)$ where:

$$
V=\left(\begin{array}{cc}
1 & 0 \\
x & 1
\end{array}\right) \in \mathrm{GL}_{2}(D), \quad W=\left(\begin{array}{cc}
0 & 1 \\
-1 & x
\end{array}\right) \in \mathrm{GL}_{2}(D) .
$$

In particular, the matrices $R$ and $R^{\prime}$ are not equivalent ([15]). This example shows that even if a matrix with entries in $D$ is generally no more equivalent to its Smith normal form as it is the case for a principal ideal domain (e.g., $k[x]$ ), Serre's reduction can sometimes be useful to check whether or not two finitely presented modules are isomorphic, i.e., to check non-equivalence of linear systems.

Finally, let us study the converse of Corollary 5 .

Corollary 6. Let $R \in D^{q \times p}$ be a full row rank matrix and $V \in \mathrm{GL}_{q}(D)$ and $W \in \mathrm{GL}_{p}(D)$ two matrices such that $V R W=\operatorname{diag}\left(I_{r}, Q_{2}\right)$, where $0 \leq r \leq q-1$ and $Q_{2} \in D^{(q-r) \times(p-r)}$. Then, there exist $U \in \mathrm{GL}_{p+q-r}(D)$ and $\Lambda \in D^{q \times(q-r)}$ which admits a left inverse $\Gamma \in D^{(q-r) \times q}$ such that $\left(\begin{array}{ll}R & -\Lambda\end{array}\right) U=\left(\begin{array}{ll}I_{q} & 0\end{array}\right)$ and $\operatorname{ker}_{D}\left(. Q_{1}\right)$ is a free left $D$-module of rank $r$, where $W=\left(\begin{array}{ll}Q_{3} & Q_{1}\end{array}\right)$, $Q_{3} \in D^{p \times r}$ and $Q_{1} \in D^{p \times(p-r)}$. In particular, $\Lambda \in D^{q \times(q-r)}$ can be chosen to be the submatrix of $V^{-1}=\left(\begin{array}{ll}\Phi & \Lambda\end{array}\right)$, where $\Phi \in D^{q \times r}$. 
Proof. If $W=\left(\begin{array}{ll}Q_{3} & Q_{1}\end{array}\right)$, where $Q_{3} \in D^{p \times r}$ and $Q_{1} \in D^{p \times(p-r)}$, and $V^{-1}=\left(\begin{array}{ll}X_{1} & \Lambda\end{array}\right)$, where $X_{1} \in D^{q \times r}$ and $\Lambda \in D^{q \times(q-r)}$, then $V R W=\operatorname{diag}\left(I_{r}, Q_{2}\right)$ yields:

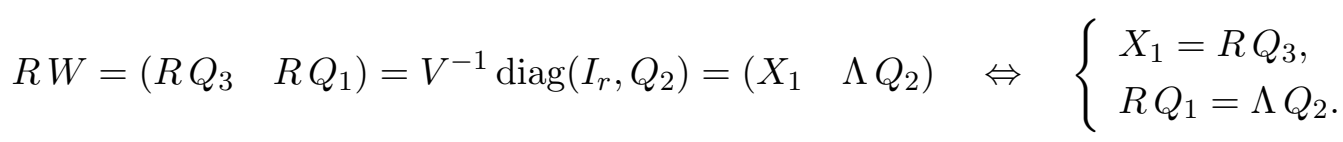

Now, if we write $V=\left(\begin{array}{ll}V_{1}^{T} & V_{2}^{T}\end{array}\right)^{T}$, where $V_{1} \in D^{r \times q}$ and $V_{2} \in D^{(q-r) \times q}$, then the relation $V^{-1} V=I_{q}$ yields $R Q_{3} V_{1}+\Lambda V_{2}=I_{q}$, i.e., $R\left(Q_{3} V_{1}\right)-\Lambda\left(-V_{2}\right)=I_{q}$, which shows that the matrix $P=\left(\begin{array}{ll}R & -\Lambda\end{array}\right) \in D^{q \times(p+q-r)}$ admits the right-inverse $S=\left(\left(Q_{3} V_{1}\right)^{T}-V_{2}^{T}\right)^{T}$ and the left $D$-module $E=D^{1 \times(p+q-r)} /\left(D^{1 \times q} P\right)$ is stably free of rank $r$. The relation $V V^{-1}=I_{q}$ implies that $V_{2} \Lambda=I_{q-r}$, which shows that the matrix $\Lambda$ admits the left-inverse $V_{2}$ over $D$. Now if $W^{-1}=\left(\begin{array}{ll}Y_{3}^{T} & Y_{1}^{T}\end{array}\right)^{T}$, where $Y_{3} \in D^{r \times p}$ and $Y_{1} \in D^{(p-r) \times p}$, then the identity $W^{-1} W=I_{p}$ particularly gives $Y_{1} Q_{3}=0$ and $Y_{1} Q_{1}=I_{p-r}$, and thus

$$
\left(\begin{array}{cc}
R & -\Lambda \\
Y_{1} & 0
\end{array}\right)\left(\begin{array}{cc}
Q_{3} V_{1} & Q_{1} \\
-V_{2} & Q_{2}
\end{array}\right)=\left(\begin{array}{cc}
I_{q} & 0 \\
0 & I_{p-r}
\end{array}\right)=I_{p+q-r}
$$

which proves the existence of $U \in \mathrm{GL}_{p+q-r}(D)$ satisfying $\left(\begin{array}{ll}R & -\Lambda\end{array}\right) U=\left(\begin{array}{ll}I_{q} & 0\end{array}\right)$. Finally, the identities $W W^{-1}=I_{p}$ and $W^{-1} W=I_{p}$ are equivalent to the split short exact sequence (31), which shows that $\operatorname{ker}_{D}\left(. Q_{1}\right)=D^{1 \times r} Y_{3} \cong D^{1 \times r}$ and proves that $\operatorname{ker}_{D}\left(. Q_{1}\right)$ is a free left $D$-module of rank $r$.

Corollaries 5 and 6 generalize Theorem 1 to matrices which are not necessarily square over a more general class of rings $D$.

We finally state a straightforward application of Theorem 5 to the study of the so-called doubly coprime factorizations of a transfer matrix $P$ (see, e.g., [2, 13, 30]). We slightly change the notation of the base ring from $D$ to $A$ in order to use the standard notation $P=D^{-1} N$ chosen in control theory. We recall that an integral domain is a commutative domain.

Corollary 7. Let $A$ be an integral domain, $K=\{d / n \mid d \in A \backslash\{0\}, n \in A\}$ its quotient field and $P=D^{-1} N=\widetilde{N} \widetilde{D}^{-1}$ a doubly coprime factorization of the transfer matrix $P \in K^{q \times r}$, where $D \in A^{q \times q}, N \in A^{q \times r}, \widetilde{D} \in A^{r \times r}$ and $\widetilde{N} \in A^{q \times r}$, namely, there exist $X \in A^{q \times q}, Y \in A^{r \times q}$, $\widetilde{X} \in A^{r \times r}$ and $\widetilde{Y} \in A^{r \times q}$ such that:

$$
\left(\begin{array}{cc}
D & -N \\
-\widetilde{Y} & \tilde{X}
\end{array}\right)\left(\begin{array}{cc}
X & \widetilde{N} \\
Y & \widetilde{D}
\end{array}\right)=I_{p}
$$

Then, we have the following A-isomorphism:

$$
M=A^{1 \times q} /\left(A^{1 \times q} D\right) \cong L=A^{1 \times r} /\left(A^{1 \times r} \widetilde{D}\right) .
$$

Moreover, if $N$ admits a left-inverse over $A$ and $q \geq r$, then $\operatorname{ker}_{A}(. \tilde{N})$ is a stably free A-module of rank $q-r$. If $\operatorname{ker}_{A}(. \widetilde{N})$ is a free of rank $q-r$, then there exists a matrix $Z \in D^{q \times(q-r)}$ such

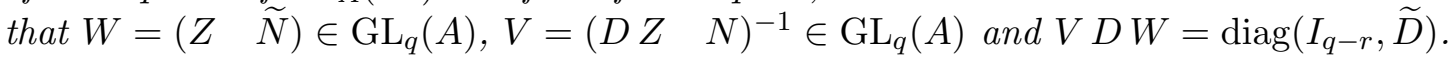

More generally, if we set

$\mathrm{RR} \mathrm{n}^{\circ} 7214$

$$
1 \leq s \leq r, \quad\left\{\begin{array}{lll}
N=\left(\begin{array}{ll}
N_{1} & N_{2}
\end{array}\right), & N_{1} \in A^{q \times(r-s)}, & N_{2} \in A^{q \times s}, \\
\widetilde{D}=\left(\begin{array}{c}
\widetilde{D}_{1} \\
\widetilde{D}_{2}
\end{array}\right), & \widetilde{D}_{1} \in A^{(r-s) \times r} & \widetilde{D}_{2} \in A^{s \times r},
\end{array}\right.
$$


then we have the following A-isomorphism:

$$
M_{s}=A^{1 \times(q+r-s)} /\left(A^{1 \times q}\left(D-N_{1}\right)\right) \cong L_{s}=A^{1 \times r} /\left(A^{1 \times s} \widetilde{D}_{2}\right) .
$$

Moreover, if $N_{2}$ admits a left-inverse over $A$ and $q \geq s$, then $\operatorname{ker}_{A}\left(.\left(\widetilde{N}^{T} \quad \widetilde{D}_{1}^{T}\right)^{T}\right)$ is a stably free $A$-module of rank $q-s$. If $\operatorname{ker}_{A}\left(.\left(\widetilde{N}^{T} \widetilde{D}_{1}^{T}\right)^{T}\right)$ is a free A-module of rank $q-s$, then there exists

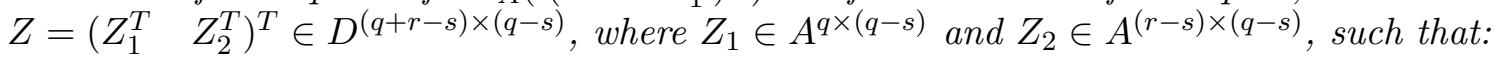

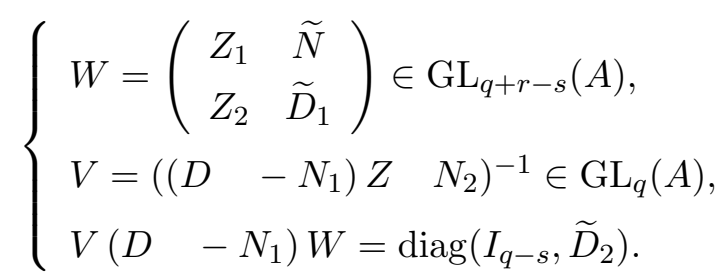

Example 19. Let us consider the following rational transfer ([40])

$$
P=\frac{1}{\left(x_{1}-x_{2}-1\right)}\left(\begin{array}{cc}
-x_{2}^{2}-x_{1} x_{2}+x_{1}+x_{2}-1 & -2 x_{2} \\
x_{2}^{2}+x_{1} x_{2}-x_{1}+x_{2} & x_{1}+x_{2}+1
\end{array}\right) \in K^{2 \times 2},
$$

where $K=\mathbb{R}\left(x_{1}, x_{2}, x_{3}\right)$ is the quotient field of the integral domain $A=\mathbb{R}\left[x_{1}, x_{2}, x_{3}\right]$. Using the algorithms for the computation of doubly coprime factorizations of rational transfer matrices (when they exist) described in [13] and implemented in the package QuilenSusLin ([13]), we obtain that $P$ admits the doubly coprime factorization $P=D^{-1} N=\widetilde{N} \widetilde{D}^{-1}$, where:

$$
\begin{aligned}
& \left\{\begin{aligned}
D & =\left(\begin{array}{cc}
x_{1}+x_{2}+1 & x_{1}+x_{2}-1 \\
x_{2}^{2}+x_{1} x_{2}-x_{1}+x_{2} & x_{2}^{2}+x_{1} x_{2}-x_{1}-x_{2}+1
\end{array}\right), \\
N & =\left(\begin{array}{cc}
1 & x_{1}+x_{2}+1 \\
0 & x_{2}^{2}+x_{1} x_{2}-x_{1}+x_{2}-1
\end{array}\right), \\
X & =\left(\begin{array}{ll}
0 & 1 \\
0 & 0
\end{array}\right), \\
Y & =\left(\begin{array}{cc}
-1 & 0 \\
0 & 1
\end{array}\right), \\
\widetilde{D} & =\left(\begin{array}{cc}
-2 \\
-2 x_{2}+1 & -2 x_{1}+2 x_{2}+2
\end{array}\right) \\
\widetilde{N} & =\left(\begin{array}{cc}
-2 x_{2}+2 & 4 x_{2} \\
-1
\end{array}\right) \\
\widetilde{X} & =\frac{1}{4}\left(\begin{array}{cc}
2 & 0 \\
0 & 1
\end{array}\right), \\
\widetilde{Y} & =\frac{1}{4}\left(\begin{array}{cc}
0 & 0 \\
1 & 1
\end{array}\right) .
\end{aligned}\right.
\end{aligned}
$$

For more details, see [13. Hence, we obtain the following two $A$-isomorphisms

$$
\begin{aligned}
M=A^{1 \times 2} /\left(A^{1 \times 2} D\right) & \cong L=A^{1 \times 2} /\left(A^{1 \times 2} \widetilde{D}\right), \\
M_{1}=A^{1 \times 3} /\left(A^{1 \times 2}\left(D-N_{1}\right)\right) & \cong L_{1}=A^{1 \times 2} /\left(A \widetilde{D}_{2}\right),
\end{aligned}
$$

$\mathrm{RR} \mathrm{n}^{\circ} 7214$ 
where $N_{1}=\left(\begin{array}{ll}1 & 0\end{array}\right)^{T}$ and $\widetilde{D}_{2}=\left(\begin{array}{ll}-2 x_{2}+1 & -2 x_{1}+2 x_{2}+2\end{array}\right)$. Finally, since the matrix $N$ (resp., $\left.N_{2}=\left(x_{1}+x_{2}+1 \quad x_{2}^{2}+x_{1} x_{2}-x_{1}+x_{1}-1\right)^{T}\right)$ does not admit a left-inverse over $A$, we cannot conclude on the possible equivalence of the matrices $\left(D-N_{1}\right)$ and $\operatorname{diag}\left(1, \widetilde{D}_{2}\right)$.

Corollary 7 has particularly interesting applications to stabilization problems studied in control theory when $A=R H_{\infty}, H^{\infty}\left(\mathbb{C}_{+}\right), H^{\infty}(\mathbb{D}), \mathcal{A}, \hat{\mathcal{A}}, W_{+}, \ldots$ (see [30] and the references therein). They will be developed in a forthcoming publication. Finally, if $M_{s}$ is a torsion-free $A$-module, then the presentation matrix $R_{s}=\left(\begin{array}{ll}D & -N_{1}\end{array}\right)$ of the $A$-module $M_{s}$ corresponds to the transfer matrix $y=P u$ where the last $s$ components of the input $u$, namely, $u_{r+1-s}, \ldots, u_{r}$, are set to 0 . For more details, see [30].

\section{Conclusion}

In this paper, we have studied a constructive version of Serre's reduction ([39]) and explained its main interests in mathematical systems theory. Moreover, we have pointed out interesting connections between Serre's reduction and the problem of recognizing when a matrix over a commutative polynomial ring with coefficients in a field is equivalent to its Smith normal form. As we have shown on different explicit examples, Serre's reduction of rather complicated linear functional systems can sometimes be extremely simple and useful for the investigation of their structural properties, their closed-form integration as well as numerical analysis issues.

Serre's reduction of linear systems of partial differential equations with varying coefficients is studied in more details in [10] and particularly in the case where $\operatorname{ext}_{D}^{1}(M, D)$ is a so-called holonomic right $D$-module.

Finally, the different algorithms explained in this paper will be soon available in the package SERRE ([11]) and a large library of examples will illustrate its functionalities and interest of Serre's reduction (for more examples of Serre's reductions, see also [9]).

\section{Acknowledgment}

This paper is dedicated to the memory of our dear friend N. K. Bose. The authors would like to thank T. Cluzeau and D. Robertz for pointing out to us typos in the last version of the paper.

\section{Appendix: Worked examples using Maple}

We now demonstrate how Serre's reductions can be explicitly obtained on explicit examples using the package OreModules ([7]). These different steps of Serre's reduction will soon be implemented in the package SERRE ([11]).

We first need to call the package OreModules and the Maple library linalg:

$>$ with(OreModules):

$>$ with(linalg):

In the previous sections, we have used the notation $D$ for a ring of functional operators. Since this notation is protected in Maple, we shall now use the notation $A$ instead of $D$. 
Example 20. We consider the linear system of ordinary differential time-delay equations defining a wind tunnel model studied in [23]:

$$
\left\{\begin{array}{l}
\dot{x}_{1}(t)+a x_{1}(t)-k a x_{2}(t-h)=0 \\
\dot{x}_{2}(t)-x_{3}(t)=0 \\
\dot{x}_{3}(t)+\omega^{2} x_{2}(t)+2 \zeta \omega x_{3}(t)-\omega^{2} u(t)=0
\end{array}\right.
$$

The presentation matrix of (37) is defined by

$>\mathrm{R}:=\operatorname{matrix}(3,4,[\mathrm{~d}+\mathrm{a}, \mathrm{k} * \mathrm{a} * \operatorname{delta}, 0,0,0, \mathrm{~d},-1,0,0$, omega^2, $\mathrm{d}+2 * x i *$ omega,
$>\quad$-omega^2]);

$$
R:=\left[\begin{array}{cccc}
d+a & k a \delta & 0 & 0 \\
0 & d & -1 & 0 \\
0 & \omega^{2} & d+2 \zeta \omega & -\omega^{2}
\end{array}\right]
$$

where $a, k, \omega$ and $\zeta$ are constant parameters of the system. Let us now introduce the commutative polynomial $\operatorname{ring} A=\mathbb{Q}(a, k, \omega, \zeta)[d, \delta]$ of ordinary differential time-delay operators, i.e., $d y(t)=\dot{y}(t)$ and $\delta y(t)=y(t-h)$,

$>A:=$ DefineOreAlgebra $\left(\operatorname{diff}=[\mathrm{d}, \mathrm{t}]\right.$, dual_shift $^{\prime}=[\operatorname{delta}, \mathrm{s}]$, polynom $=[\mathrm{t}, \mathrm{s}]$,

$>\mathrm{comm}=[\mathrm{a}, \mathrm{k}$, omega, $\mathrm{xi}])$ :

and the finitely presented $A$-module $M=A^{1 \times 4} /\left(A^{1 \times 3} R\right)$. Let us show how to use Theorem 5 to find Serre's reduction of the corresponding differential time-delay system. As explained in Section 3 , the hypothesis of Theorem 5 can completely be checked when the $A$-module $\operatorname{ext}_{A}^{1}(M, A)=A^{3} /\left(R A^{4}\right)$ is 0 -dimensional, i.e., a finite-dimensional $\mathbb{Q}(a, k, \omega, \zeta)$-vector space (see also Remark 3). Let us check whether or not this hypothesis is fulfilled using the command DimensionRat of OREMODULES:

$$
>\text { DimensionRat (transpose }(R), A) \text {; }
$$

0

Now, we can compute a finite basis of the $\mathbb{Q}(a, k, \omega, \zeta)$-vector space $\operatorname{ext}_{A}^{1}(M, A)$ using the function KBasis of OreModules:

$>$ KBasis(transpose $(R), A)$;

\section{$\left[\lambda_{1}\right]$}

We obtain that $\operatorname{ext}_{A}^{1}(M, A)$ is a 1-dimensional $\mathbb{Q}(a, k, \omega, \zeta)$-vector space defined by the residue class $\tau(\Lambda)$ of the column vector $\Lambda=\left(\begin{array}{lll}1 & 0 & 0\end{array}\right)^{T}$ in $\operatorname{ext}_{A}^{1}(M, A)$. Hence, we can consider the column vector $\Lambda$ defined by:

$>\operatorname{Lambda}:=\operatorname{evalm}([[1],[0],[0]])$;

$$
\Lambda:=\left[\begin{array}{l}
1 \\
0 \\
0
\end{array}\right]
$$

Let us now define the new matrix $P=\left(\begin{array}{ll}R & -\Lambda\end{array}\right) \in D^{4 \times 5}$ :

$>\mathrm{L}:=\operatorname{augment}(\mathrm{R},-$ Lambda $)$; 


$$
P:=\left[\begin{array}{ccccc}
d+a & -k a \delta & 0 & 0 & -1 \\
0 & d & -1 & 0 & 0 \\
0 & \omega^{2} & d+2 \zeta \omega & -\omega^{2} & 0
\end{array}\right]
$$

Let us check whether or not the matrix $P$ admits a right-inverse over $A$ :

$>\mathrm{S}:=$ RightInverse $(\mathrm{P}, \mathrm{A})$;

$$
S:=\left[\begin{array}{ccc}
0 & 0 & 0 \\
0 & 0 & 0 \\
0 & -1 & 0 \\
0 & -\frac{d+2 \zeta \omega}{\omega^{2}} & -\omega^{-2} \\
-1 & 0 & 0
\end{array}\right]
$$

According to Theorem 4 and the Quillen-Suslin theorem (see 2 of Theorem 3), the $A$-module $E=A^{1 \times 5} /\left(A^{1 \times 3} P\right)$ is stably free, and thus, free of rank 2 . Let us compute an injective parametrization of the free $A$-module $E$ :

$>\mathrm{Q}:=$ MinimalParametrization $(\mathrm{P}, \mathrm{A})$;

$$
Q:=\left[\begin{array}{cc}
-1 & 0 \\
0 & \omega^{2} \\
0 & \omega^{2} d \\
0 & \omega^{2}+d^{2}+2 d \zeta \omega \\
-d-a & -\omega^{2} k a \delta
\end{array}\right]
$$

We get $\operatorname{ker}_{A}(. Q)=A^{1 \times 3} P$, i.e., $Q$ is a parametrization of $E$. Let us check whether or $Q$ admits a left-inverse over $A$ :

$>\quad \mathrm{T}:=\operatorname{LeftInverse}(\mathrm{Q}, \mathrm{A})$;

$$
T:=\left[\begin{array}{ccccc}
-1 & 0 & 0 & 0 & 0 \\
0 & \omega^{-2} & 0 & 0 & 0
\end{array}\right]
$$

The matrix $Q$ is then an injective parametrization of $E$ and the residue classes of the rows of $T$ define a basis of the free $A$-module $E$ of rank 2. Let us now write $Q=\left(\begin{array}{ll}Q_{1}^{T} \quad Q_{2}^{T}\end{array}\right)^{T}$, where $Q_{1} \in A^{4 \times 2}$ is the matrix defined by

$>Q_{-} 1:=\operatorname{submatrix}(Q, 1 \ldots 4,1 \ldots 2)$;

$$
Q_{1}:=\left[\begin{array}{cc}
-1 & 0 \\
0 & \omega^{2} \\
0 & \omega^{2} d \\
0 & \omega^{2}+d^{2}+2 d \zeta \omega
\end{array}\right]
$$

and $Q_{2} \in A^{1 \times 3}$ is given by:

$>$ Q2:=submatrix $(Q, 5 \ldots 5,1 \ldots 2)$;

$$
Q_{2}:=\left[\begin{array}{ll}
-d-a & -\omega^{2} k a \delta
\end{array}\right]
$$





$A$-module $\mathcal{F}$ (see Corollary 3 ).

Let us now check whether or not the matrix $R$ is equivalent to the block-diagonal matrix $\bar{R}=\operatorname{diag}\left(I_{2}, Q_{2}\right)$. The column vector $\Lambda$ admits the following left-inverse:

$>$ LeftInverse(Lambda, A);

$$
\left[\begin{array}{lll}
1 & 0 & 0
\end{array}\right]
$$

Let us check whether or not the $A$-module $\operatorname{ker}_{A}\left(. Q_{1}\right)$ is free of rank 2 :

$>\mathrm{K}:=\operatorname{SyzygyModule}\left(\mathrm{Q}_{-} 1, \mathrm{~A}\right)$;

$$
K:=\left[\begin{array}{cccc}
0 & \omega^{2} & d+2 \zeta \omega & -\omega^{2} \\
0 & d & -1 & 0
\end{array}\right]
$$

Since the matrix $K$ satisfies $\operatorname{ker}_{A}\left(. Q_{1}\right)=A^{1 \times 2} K$ and $K$ has full row rank

$>$ SyzygyModule $(\mathrm{K}, \mathrm{A})$;

$$
\operatorname{INJ}(2)
$$

we obtain that $\operatorname{ker}_{A}\left(. Q_{1}\right)=A^{1 \times 2} K \cong A^{1 \times 2}$ is a free $A$-module of rank 2 . Hence, the hypotheses of Corollary 5 are fulfilled. Let us now complete the matrix $Q_{1}$ to a unimodular matrix $W=$ $\left(\begin{array}{ll}Q_{3} & Q_{1}\end{array}\right) \in \mathrm{GL}_{4}(A)$. We can take:

$>$ Q_3:=RightInverse $(K, A)$;

$$
Q_{3}:=\left[\begin{array}{cc}
0 & 0 \\
0 & 0 \\
0 & -1 \\
-\omega^{-2} & -\frac{d+2 \zeta \omega}{\omega^{2}}
\end{array}\right]
$$

The matrix $W=\left(\begin{array}{ll}Q_{3} & Q_{1}\end{array}\right)$ is then defined by:

$>\mathrm{W}:=$ augment (Q_3, Q_1);

$$
W:=\left[\begin{array}{cccc}
0 & 0 & -1 & 0 \\
0 & 0 & 0 & \omega^{2} \\
0 & -1 & 0 & \omega^{2} d \\
-\omega^{-2} & -\frac{d+2 \zeta \omega}{\omega^{2}} & 0 & \omega^{2}+d^{2}+2 d \zeta \omega
\end{array}\right]
$$

Let us check again that the matrix $W$ is unimodular, i.e., $W \in \mathrm{GL}_{4}(A)$ :

$>$ W_inv:=inverse (W);

$$
W \_i n v:=\left[\begin{array}{cccc}
0 & \omega^{2} & d+2 \zeta \omega & -\omega^{2} \\
0 & d & -1 & 0 \\
-1 & 0 & 0 & 0 \\
0 & \omega^{-2} & 0 & 0
\end{array}\right]
$$

According to Corollary 5, the matrix $X=\left(\begin{array}{ll}R Q_{3} & \Lambda\end{array}\right)$ defined by 
$>\mathrm{X}:=\operatorname{augment}(\operatorname{Mult}(\mathrm{R}, \mathrm{Q} 3, \mathrm{~A})$, Lambda) ;

$$
X:=\left[\begin{array}{lll}
0 & 0 & 1 \\
0 & 1 & 0 \\
1 & 0 & 0
\end{array}\right]
$$

is unimodular, i.e., $X \in \mathrm{GL}_{3}(A), V=X^{-1}=X$ and the matrix $R$ is equivalent to the blockdiagonal matrix $V R W=\operatorname{diag}\left(I_{2}, Q_{2}\right)$ :

$>\operatorname{Mult}(\mathrm{X}, \mathrm{R}, \mathrm{W}, \mathrm{A})$;

$$
\left[\begin{array}{cccc}
1 & 0 & 0 & 0 \\
0 & 1 & 0 & 0 \\
0 & 0 & -d-a & -\omega^{2} k a \delta
\end{array}\right]
$$

The linear differential time-delay system (37) is then equivalent to the following single-input single-output differential time-delay system:

$$
\dot{z}(t)+a z(t)+\omega^{2} k a v(t-h)=0 .
$$

Then, we can then easily study the structural properties of the latter simple differential timedelay system and of its associated $A$-module (e.g., torsion-free, $\delta$-free), and thus those of (37).

Example 21. We consider the model (13) of a string with an interior mass studied in Examples 4,10 and 13 (see also [25]). We first introduce the commutative polynomial ring $A=\mathbb{Q}\left(\eta_{1}, \eta_{2}\right)\left[d, \sigma_{1}, \sigma_{2}\right]$ of ordinary differential incommensurable time-delay operators, where $d y(t)=\dot{y}(t)$ and $\sigma_{i} y(t)=y\left(t-h_{i}\right)$ for all $i=1,2$.

$>A:=$ DefineOreAlgebra (diff $=[d, t]$, dual_shift $=[\operatorname{sigma}[1], x[1]]$,

$>$ dual_shift $=[\operatorname{sigma}[2], x[2]]$, polynom $=[t, x[1], x[2]], \operatorname{comm}=[$ eta[1], eta[2] $]):$

The presentation matrix $R \in A^{4 \times 6}$ of $(13)$ is defined by:

$>\mathrm{R}:=\operatorname{matrix}(4,6,[1,1,-1,-1,0,0, \mathrm{~d}+\mathrm{eta}[1], \mathrm{d}-\mathrm{eta}[1],-\mathrm{eta}[2]$, eta $[2], 0,0$,

$\left.\left.>\operatorname{sigma}[1]^{\wedge} 2,1,0,0,-\operatorname{sigma}[1], 0,0,0,1, \operatorname{sigma}[2]^{\wedge} 2,0,-\operatorname{sigma}[2]\right]\right)$;

$$
R:=\left[\begin{array}{cccccc}
1 & 1 & -1 & -1 & 0 & 0 \\
d+\eta_{1} & d-\eta_{1} & -\eta_{2} & \eta_{2} & 0 & 0 \\
\sigma_{1}^{2} & 1 & 0 & 0 & -\sigma_{1} & 0 \\
0 & 0 & 1 & \sigma_{2}{ }^{2} & 0 & -\sigma_{2}
\end{array}\right]
$$

Let us illustrate Algorithm 1 with this example. As explained in Section 3 , the hypothesis of Theorem 5 can be completely checked when the $A$-module $\operatorname{ext}_{A}^{1}(M, A)=A^{3} /\left(R A^{4}\right)$ is 0 dimensional, i.e., is a finite-dimensional $\mathbb{Q}\left(\eta_{1}, \eta_{2}\right)$-vector space. Let us check whether or not this hypothesis is fulfilled using the command DimensionRat of OREMODULES:

$>$ DimensionRat (transpose $(R), A)$;

Now, we can compute a finite basis of the $\mathbb{Q}\left(\eta_{1}, \eta_{2}\right)$-vector space $\operatorname{ext}_{A}^{1}(M, A)$ using the command KBasis of OreMODULES:

$>\operatorname{KBasis}(\operatorname{transpose}(\mathrm{R}), \mathrm{A})$; 


\section{$\left[\lambda_{4}\right]$}

We obtain that the $A$-module $\operatorname{ext}_{A}^{1}(M, A)=A^{3} /\left(R A^{4}\right)$ is a 1 -dimensional $\mathbb{Q}\left(\eta_{1}, \eta_{2}\right)$-vector space of basis the residue class $\tau(\Lambda)$ of the column vector $\Lambda=\left(\begin{array}{lll}0 & 0 & 1\end{array}\right)^{T} \operatorname{in}_{\operatorname{ext}_{A}^{1}(M, A) \text {. Hence, let }}$ us consider the column vector $\Lambda=\left(\begin{array}{lll}0 & 0 & 1\end{array}\right)^{T}$

$>$ Lambda:=evalm([[0],[0],[0],[1]]);

$$
\Lambda:=\left[\begin{array}{l}
0 \\
0 \\
0 \\
1
\end{array}\right]
$$

the matrix $P=\left(\begin{array}{ll}R & -\Lambda\end{array}\right)$ defined by

$>P:=\operatorname{augment}(\mathrm{R},-\operatorname{evalm}([[0],[0],[0],[1]]))$;

$$
P:=\left[\begin{array}{ccccccc}
1 & 1 & -1 & -1 & 0 & 0 & 0 \\
d+\eta_{1} & d-\eta_{1} & -\eta_{2} & \eta_{2} & 0 & 0 & 0 \\
\sigma_{1}{ }^{2} & 1 & 0 & 0 & -\sigma_{1} & 0 & 0 \\
0 & 0 & 1 & \sigma_{2}{ }^{2} & 0 & -\sigma_{2} & -1
\end{array}\right]
$$

and the $A$-module $E=A^{1 \times 7} /\left(A^{1 \times 4} P\right)$. Let us now check whether or not the $A$-module $E$ is free. According to Theorem 4 , the full row rank matrix $P$ presents a stably free $A$-module $E$ iff $P$ admits a right-inverse over $A$. Let us check this point:

$>$ RightInverse $(\mathrm{P}, \mathrm{A})$;

$$
\left[\begin{array}{cccc}
0 & 0 & -1 & 0 \\
0 & 0 & 1 & 0 \\
-1 / 2 & -1 / 2 \eta_{2}^{-1} & -\frac{\eta_{1}}{\eta_{2}} & 0 \\
-1 / 2 & 1 / 2 \eta_{2}^{-1} & \frac{\eta_{1}}{\eta_{2}} & 0 \\
0 & 0 & -\sigma_{1} & 0 \\
-1 / 2 \sigma_{2} & 1 / 2 \frac{\sigma_{2}}{\eta_{2}} & \frac{\eta_{1} \sigma_{2}}{\eta_{2}} & 0 \\
-1 / 2 & -1 / 2 \eta_{2}^{-1} & -\frac{\eta_{1}}{\eta_{2}} & -1
\end{array}\right]
$$

We obtain that $E$ is a stably free $A$-module, and thus, a free of rank 2 by the Quillen-Suslin theorem (2 of Theorem 3). Let us compute a minimal parametrization of the $A$-module $E$ :

$>\mathrm{Q}:=$ MinimalParametrization $(\mathrm{P}, \mathrm{A})$; 


$$
Q:=\left[\begin{array}{ccc}
-2 \eta_{2} & \eta_{2} \sigma_{1} & 0 \\
0 & -\eta_{2} \sigma_{1} & 0 \\
-d-\eta_{1}-\eta_{2} & \sigma_{1} \eta_{1} & 0 \\
\eta_{1}-\eta_{2}+d & -\sigma_{1} \eta_{1} & 0 \\
-2 \eta_{2} \sigma_{1} & -\eta_{2}+\eta_{2} \sigma_{1}^{2} & 0 \\
\eta_{1} \sigma_{2}-\sigma_{2} \eta_{2}+\sigma_{2} d & -\sigma_{1} \eta_{1} \sigma_{2} & 1 \\
-d-\eta_{1}-\eta_{2} & \sigma_{1} \eta_{1} & -\sigma_{2}
\end{array}\right]
$$

Hence, we get $\operatorname{ker}_{A}(. Q)=A^{1 \times 4} P$ or equivalently $E \cong A^{1 \times 7} Q$. Let us check whether or not this parametrization is injective:

$>\mathrm{T}:=\operatorname{LeftInverse}(\mathrm{Q}, \mathrm{A})$;

$$
T:=\left[\begin{array}{ccccccc}
0 & 0 & -1 / 2 \eta_{2}{ }^{-1} & -1 / 2 \eta_{2}{ }^{-1} & 0 & 0 & 0 \\
0 & -\frac{\sigma_{1}}{\eta_{2}} & \frac{\sigma_{1}}{\eta_{2}} & \frac{\sigma_{1}}{\eta_{2}} & -\eta_{2}{ }^{-1} & 0 & 0 \\
0 & 0 & 0 & -\sigma_{2} & 0 & 1 & 0
\end{array}\right]
$$

We get $T Q=I_{3}$, i.e., $A^{1 \times 7} Q=A^{1 \times 3}$, which proves that $Q$ is an injective parametrization of $E$. Let us now write $Q=\left(\begin{array}{ll}Q_{1}^{T} & Q_{2}^{T}\end{array}\right)^{T}$, where the submatrix $Q_{1} \in A^{6 \times 3}$ is defined by

$>Q_{-} 1:=\operatorname{submatrix}(Q, 1 \ldots 6,1 \ldots 3)$;

$$
Q_{1}:=\left[\begin{array}{ccc}
-2 \eta_{2} & \eta_{2} \sigma_{1} & 0 \\
0 & -\eta_{2} \sigma_{1} & 0 \\
-d-\eta_{1}-\eta_{2} & \sigma_{1} \eta_{1} & 0 \\
\eta_{1}-\eta_{2}+d & -\sigma_{1} \eta_{1} & 0 \\
-2 \eta_{2} \sigma_{1} & -\eta_{2}+\eta_{2} \sigma_{1}^{2} & 0 \\
\eta_{1} \sigma_{2}-\sigma_{2} \eta_{2}+\sigma_{2} d & -\sigma_{1} \eta_{1} \sigma_{2} & 1
\end{array}\right]
$$

and the matrix $Q_{2} \in A^{1 \times 3}$ is defined by:

$>$ Q_2:=submatrix $(Q, 7 \ldots 7,1 \ldots 3)$;

$$
Q_{2}:=\left[-d-\eta_{1}-\eta_{2} \quad \sigma_{1} \eta_{1}-\sigma_{2}\right]
$$

According to Theorem 5 , we have $M \cong A^{1 \times 3} /\left(A Q_{2}\right)$, which, using Corollary 3 , proves again that the linear system $\operatorname{ker}_{\mathcal{F}}(R$. $)$ is equivalent to $\operatorname{ker}_{\mathcal{F}}\left(Q_{2}\right.$.), namely, 27].

Since the column vector $\Lambda$ admits a left-inverse over $A$ defined by

$>$ LeftInverse (Lambda, A);

$$
\left[\begin{array}{llll}
0 & 0 & 0 & 1
\end{array}\right]
$$

the Quillen-Suslin theorem (3 of Theorem 3 ) implies that there exist two matrices $V \in \mathrm{GL}_{4}(A)$ and $W \in \mathrm{GL}_{6}(A)$ such that $V R W=\operatorname{diag}\left(I_{3}, Q_{2}\right)$. For more details, see Corollary 5 . Let us compute such matrices $V$ and $W$ following Corollary 5 . We first need to check that $\operatorname{ker}_{A}\left(. Q_{1}\right)$ is a free $A$-module of rank 3 :

$>\mathrm{K}:=\operatorname{SyzygyModule}\left(\mathrm{Q}_{-} 1, \mathrm{~A}\right)$; 


$$
K:=\left[\begin{array}{cccccc}
1 & 1 & -1 & -1 & 0 & 0 \\
0 & -2 \eta_{1} & \eta_{1}-\eta_{2}+d & d+\eta_{2}+\eta_{1} & 0 & 0 \\
0 & -1+\sigma_{1}{ }^{2} & -\sigma_{1}{ }^{2} & -\sigma_{1}{ }^{2} & \sigma_{1} & 0
\end{array}\right]
$$

Then, we get $\operatorname{ker}_{A}\left(. Q_{1}\right)=A^{1 \times 3} K$. Moreover, $K$ has full row rank since:

$>$ SyzygyModule $(\mathrm{K}, \mathrm{A})$;

$$
\operatorname{INJ}(3)
$$

Hence, we get $A^{1 \times 3} K \cong A^{1 \times 3}$, a fact proving that $\operatorname{ker}_{A}\left(. Q_{1}\right)$ is a free $A$-module of rank 3 . Let us now compute a matrix $Q_{3} \in A^{6 \times 3}$ such that $W=\left(\begin{array}{ll}Q_{3} & Q_{1}\end{array}\right) \in \mathrm{GL}_{6}(A)$. We can take:

$>$ Q_3:=RightInverse $(\mathrm{K}, \mathrm{A})$;

$$
Q_{3}:=\left[\begin{array}{ccc}
1 & 0 & 1 \\
0 & 0 & -1 \\
0 & -1 / 2 \eta_{2}^{-1} & \frac{\eta_{1}}{\eta_{2}} \\
0 & 1 / 2 \eta_{2}^{-1} & -\frac{\eta_{1}}{\eta_{2}} \\
0 & 0 & \sigma_{1} \\
0 & 0 & 0
\end{array}\right]
$$

Then, the matrix $W=\left(\begin{array}{ll}Q_{3} & Q_{1}\end{array}\right)$ defined by

$>\mathrm{W}:=$ augment (Q_3, Q_1);

$$
W:=\left[\begin{array}{cccccc}
1 & 0 & 1 & -2 \eta_{2} & \eta_{2} \sigma_{1} & 0 \\
0 & 0 & -1 & 0 & -\eta_{2} \sigma_{1} & 0 \\
0 & -1 / 2 \eta_{2}^{-1} & \frac{\eta_{1}}{\eta_{2}} & -d-\eta_{1}-\eta_{2} & \sigma_{1} \eta_{1} & 0 \\
0 & 1 / 2 \eta_{2}^{-1} & -\frac{\eta_{1}}{\eta_{2}} & \eta_{1}-\eta_{2}+d & -\sigma_{1} \eta_{1} & 0 \\
0 & 0 & \sigma_{1} & -2 \eta_{2} \sigma_{1} & -\eta_{2}+\eta_{2} \sigma_{1}^{2} & 0 \\
0 & 0 & 0 & \eta_{1} \sigma_{2}-\sigma_{2} \eta_{2}+\sigma_{2} d & -\sigma_{1} \eta_{1} \sigma_{2} & 1
\end{array}\right]
$$

is invertible over $A$, i.e., $W \in \mathrm{GL}_{6}(A)$, and its inverse $W^{-1} \in A^{6 \times 6}$ is defined by:

$$
\begin{aligned}
& >\text { W_inv }:=\operatorname{inverse(W);} \\
& W \_i n v:=\left[\begin{array}{cccccc}
1 & 1 & -1 & -1 & 0 & 0 \\
0 & -2 \eta_{1} & \eta_{1}-\eta_{2}+d & d+\eta_{2}+\eta_{1} & 0 & 0 \\
0 & -1+\sigma_{1}{ }^{2} & -\sigma_{1}{ }^{2} & -\sigma_{1}{ }^{2} & \sigma_{1} & 0 \\
0 & 0 & -1 / 2 \eta_{2}{ }^{-1} & -1 / 2 \eta_{2}{ }^{-1} & 0 & 0 \\
0 & -\frac{\sigma_{1}}{\eta_{2}} & \frac{\sigma_{1}}{\eta_{2}} & \frac{\sigma_{1}}{\eta_{2}} & -\eta_{2}{ }^{-1} & 0 \\
0 & -\frac{\sigma_{1}{ }^{2} \eta_{1} \sigma_{2}}{\eta_{2}} & 1 / 2 \frac{\sigma_{2}\left(2 \sigma_{1}{ }^{2} \eta_{1}+\eta_{1}-\eta_{2}+d\right)}{\eta_{2}} & 1 / 2 \frac{\sigma_{2}\left(2 \sigma_{1}{ }^{2} \eta_{1}+\eta_{1}-\eta_{2}+d\right)}{\eta_{2}} & -\frac{\sigma_{1} \eta_{1} \sigma_{2}}{\eta_{2}} & 1
\end{array}\right]
\end{aligned}
$$

Finally, if we define the matrix $X=\left(\begin{array}{ll}R Q_{3} & \Lambda\end{array}\right)$, namely,

$$
>\quad X:=\operatorname{augment}\left(\operatorname{Mult}\left(R, Q_{-} 3, A\right),\right. \text { Lambda); }
$$




$$
X:=\left[\begin{array}{cccc}
1 & 0 & 0 & 0 \\
d+\eta_{1} & 1 & 0 & 0 \\
\sigma_{1}^{2} & 0 & -1 & 0 \\
0 & 1 / 2 \frac{-1+\sigma_{2}^{2}}{\eta_{2}} & -\frac{\eta_{1}\left(-1+\sigma_{2}^{2}\right)}{\eta_{2}} & 1
\end{array}\right]
$$

then $X$ is invertible over $A$, i.e., $V \in \mathrm{GL}_{4}(A)$, and its inverse $V=X^{-1} \in A^{4 \times 4}$ is defined by:

$>\mathrm{V}:=\operatorname{inverse}(\mathrm{X})$;

$$
V:=\left[\begin{array}{cccc}
1 & 0 & 0 & 0 \\
-d-\eta_{1} & 1 & 0 & 0 \\
\sigma_{1}{ }^{2} & 0 & -1 & 0 \\
1 / 2 \frac{\left(-1+\sigma_{2}{ }^{2}\right)\left(d+\eta_{1}+2 \sigma_{1}{ }^{2} \eta_{1}\right)}{\eta_{2}} & -1 / 2 \frac{-1+\sigma_{2}{ }^{2}}{\eta_{2}} & -\frac{\eta_{1}\left(-1+\sigma_{2}{ }^{2}\right)}{\eta_{2}} & 1
\end{array}\right]
$$

Finally, by Corollary 5, the matrix $R$ is then equivalent to the matrix $V R W=\operatorname{diag}\left(I_{3}, Q_{2}\right)$ :

$>\operatorname{Mult}(\mathrm{V}, \mathrm{R}, \mathrm{W}, \mathrm{A})$;

$$
\left[\begin{array}{cccccc}
1 & 0 & 0 & 0 & 0 & 0 \\
0 & 1 & 0 & 0 & 0 & 0 \\
0 & 0 & 1 & 0 & 0 & 0 \\
0 & 0 & 0 & -d-\eta_{1}-\eta_{2} & \sigma_{1} \eta_{1} & -\sigma_{2}
\end{array}\right]
$$

Example 22. We consider the general transmission line (14) studied in Examples 5,11 and 14 . Let $A=\mathbb{Q}(\alpha, \beta)[d x, d t]$ be the ring of partial differential operators in $d x$ and $d t$ with coefficients in the field $\mathbb{Q}(\alpha, \beta)$

$>A:=$ DefineOreAlgebra $(\operatorname{diff}=[\mathrm{dx}, \mathrm{x}]$, diff $=[\mathrm{dt}, \mathrm{t}]$, polynom $=[\mathrm{x}, \mathrm{t}]$,

$>\quad \mathrm{comm}=[\mathrm{L}, \mathrm{R}, \mathrm{C}, \mathrm{G}, \mathrm{al}$ pha, beta] $)$ :

and the presentation matrix $J \in A^{2 \times 2}$ defined by $(15)$, namely:

$>\mathrm{J}:=e v a l m([[\mathrm{dx}, \mathrm{L} * \mathrm{dt}+\mathrm{R}],[\mathrm{C} * \mathrm{dt}+\mathrm{G}, \mathrm{dx}]])$;

$$
J:=\left[\begin{array}{cc}
d x & L d t+R \\
C d t+G & d x
\end{array}\right]
$$

Let us introduce the column vector $\Lambda=\left(\begin{array}{ll}\alpha & \beta\end{array}\right)^{T}$, where $\alpha$ and $\beta$ are two arbitrary constants:

$>$ Lambda: $=\operatorname{evalm}([[\mathrm{alpha}],[$ beta $])$;

$$
\Lambda:=\left[\begin{array}{l}
\alpha \\
\beta
\end{array}\right]
$$

If we now consider the new matrix $P=\left(\begin{array}{ll}J \quad-\Lambda\end{array}\right)$, namely,

$>\mathrm{P}:=\operatorname{augment}(\mathrm{J},-$ Lambda $)$;

$$
P:=\left[\begin{array}{ccc}
d x & L d t+R & -\alpha \\
C d t+G & d x & -\beta
\end{array}\right]
$$


then we can check that $P$ does not admit a right-inverse over $A$ :

$>$ RightInverse $(\mathrm{P}, \mathrm{A})$;

Hence, the $A$-module $E=A^{1 \times 3} /\left(A^{1 \times 2} P\right)$ is generically not a stably free $A$-module. Let us determine the obstructions for $E$ to be a stably free $A$-module:

$$
\begin{aligned}
>\mathrm{pi} & :=\operatorname{map}(\text { collect, PiPolynomial (P, A), }\{\mathrm{dx}, \mathrm{dt}\}, \text { distributed); } \\
\pi & :=\left[R \beta^{2}-\alpha^{2} G+\left(-\alpha^{2} C+L \beta^{2}\right) d t, \alpha C R \beta-\alpha L \beta G+\left(-\alpha^{2} C+L \beta^{2}\right) d x\right]
\end{aligned}
$$

The obstructions are defined by the two polynomials $\pi_{1}$ and $\pi_{2}$. Let us now check whether or not there exist particular values for the arbitrary parameters $\alpha$ and $\beta$ so that $\pi_{1}$ or $\pi_{2}$ is reduced to a non-zero constant. The first entry $\pi_{1}$ of $\pi$ becomes $R \beta^{2}-G \alpha^{2}$ if we set the coefficient $-C \alpha^{2}+L \beta^{2}$ of $d t$ to 0 . The solutions of $-C \alpha^{2}+L \beta^{2}=0$ are then:

$>$ sols: $=$ solve $(\{-$ alpha^ $2 * C+L *$ beta^ 2$\},\{$ alpha, beta $\})$;

$$
\text { sols }:=\left\{\alpha=\frac{\sqrt{L C} \beta}{C}, \beta=\beta\right\},\left\{\alpha=-\frac{\sqrt{L C} \beta}{C}, \beta=\beta\right\}
$$

For the first solution of $-C \alpha^{2}+L \beta^{2}=0$, the value of $R \beta^{2}-G \alpha^{2}$ becomes:

$>$ factor $(\operatorname{subs}(\operatorname{sols}[1], R *$ beta^2-alpha^2*G));

$$
-\frac{\beta^{2}(-R C+L G)}{C}
$$

For the second solution of $-C \alpha^{2}+L \beta^{2}=0$, the value of $R \beta^{2}-G \alpha^{2}$ becomes:

$>$ factor (subs (sols[2],R*beta^2-alpha^2*G));

$$
-\frac{\beta^{2}(-R C+L G)}{C}
$$

Hence, for these two solutions, the values of $R \beta^{2}-G \alpha^{2}$ are the same and are non-zero whenever $-R C+L G \neq 0$. In other words, if we set $-C \alpha^{2}+L \beta^{2}=0$ and $-R C+L G \neq 0$, then the first obstruction $\pi_{1}$ is reduced to a non-zero constant. This particular choice is a good candidate for $P$ to admit a right-inverse.

Let us study the second obstruction $\pi_{2}$. Let us set the coefficient $-C \alpha^{2}+L \beta^{2}$ of $d x$ to 0 . Then, the parameters $\alpha$ and $\beta$ must satisfy:

$>$ sols2: $=$ solve $(\{-$ alpha^ $2 * C+L *$ beta^ 2$\},\{$ alpha, beta $\})$;

$$
\text { sols2 }:=\left\{\alpha=\frac{\sqrt{L C} \beta}{C}, \beta=\beta\right\},\left\{\alpha=-\frac{\sqrt{L C} \beta}{C}, \beta=\beta\right\}
$$

For the first solution of $-C \alpha^{2}+L \beta^{2}=0$, the constant term of $\pi_{2}$ becomes:

$>$ factor (subs (sols2[1], alpha*C*R*beta-alpha $* \mathrm{~L} *$ beta $* G))$;

$$
-\frac{\sqrt{L C} \beta^{2}(-R C+L G)}{C}
$$

For the second solution of $-C \alpha^{2}+L \beta^{2}=0$, the constant term of $\pi_{2}$ becomes:

$>$ factor (subs (sols2 [2], alpha*C*R*beta-alpha*L*beta*G)); 


$$
\frac{\sqrt{L C} \beta^{2}(-R C+L G)}{C}
$$

Therefore, if we choose the parameters $\alpha$ and $\beta$ to satisfy the conditions $-C \alpha^{2}+L \beta^{2}=0$ and $-R C+L G \neq 0$, then $\pi_{2}$ is reduced to a non-zero constant. This particular choice of conditions on $\alpha$ and $\beta$ is a good candidate for $P$ to admit a right-inverse.

Let us check whenever or not the first choice of conditions on $\alpha$ and $\beta$ is a good one (one can do the same for the second one). To simplify, we first fix $\beta=C$ and take $\alpha$ to satisfy the equation $\alpha^{2}=L C$. We then define the new ring $B=\left(\mathbb{Q}(\alpha, \beta)[\alpha] /\left(\alpha^{2}-L C\right)\right)[d x, d t]$ :

$>B:=$ DefineOreAlgebra $(\operatorname{diff}=[\mathrm{dx}, \mathrm{x}]$, diff $=[\mathrm{dt}, \mathrm{t}]$, polynom $=[\mathrm{x}, \mathrm{t}]$,

$>\quad \mathrm{comm}=[\mathrm{L}, \mathrm{R}, \mathrm{C}, \mathrm{G}, \mathrm{al}$ pha $]$, alg_relations $=[$ alpha^2-L*C] $)$ :

The column vector $\Lambda$ then becomes

$>$ Lambda_2:=subs $($ beta $=C$, evalm (Lambda));

$$
\Lambda_{2}:=\left[\begin{array}{l}
\alpha \\
C
\end{array}\right]
$$

and the matrix $P$ becomes the matrix $P_{2}$ defined by:

$>$ P_2: $=\operatorname{subs}($ beta $=C$, evalm $(P))$;

$$
P_{2}:=\left[\begin{array}{ccc}
d x & L d t+R & -\alpha \\
C d t+G & d x & -C
\end{array}\right]
$$

Let us check whether of this last matrix admits a right-inverse over $B$ :

$>\operatorname{simplify}\left(\operatorname{subs}\left(\right.\right.$ alpha^2 $2=L * C$, RightInverse $\left.\left.\left(P_{-} 2, B\right)\right)\right)$;

$$
\left[\begin{array}{cc}
-\frac{\alpha}{-R C+L G} & \frac{L}{-R C+L G} \\
-\frac{C}{-R C+L G} & \frac{\alpha}{-R C+L G} \\
-\frac{\alpha C d t+\alpha G+C d x}{C(-R C+L G)} & \frac{R C+L C d t+\alpha d x}{C(-R C+L G)}
\end{array}\right]
$$

Therefore, the $B$-module $E=B^{1 \times 3} /\left(B^{1 \times 2} P_{2}\right)$ finitely presented by the last matrix $P_{2}$ is stably free and thus free by the Quillen-Suslin theorem. Let us compute a minimal parametrization of the free $B$-module $E$ of rank 1:

$>$ Q:=MinimalParametrization(P_2,B);

$$
Q:=\left[\begin{array}{c}
-\alpha^{2} d t+\alpha d x-R C \\
-\alpha C d t-\alpha G+C d x \\
-\alpha^{2} d t^{2}-L G d t+d x^{2}-G R-C d t R
\end{array}\right]
$$

Therefore, $\operatorname{ker}_{B}(. Q)=B^{1 \times 2} P_{2}$ or, equivalently, $E \cong B^{1 \times 3} Q$. Let us check whether or not this parametrization $Q$ is injective:

$>\mathrm{T}:=$ LeftInverse $(\mathrm{Q}, \mathrm{B})$;

$$
T:=\left[\begin{array}{lll}
-\frac{C}{-\alpha^{2} G+R C^{2}} & \frac{\alpha}{-\alpha^{2} G+R C^{2}} & 0
\end{array}\right]
$$

We obtain $T Q=1$, which proves that $Q$ is an injective parametrization of the free $B$-module $E$, i.e., $E \cong D^{1 \times 3} Q=D$. In particular, the residue class $\tau(T)$ in $E$ defines a basis of the free 
$B$-module $E$ and the generators $\left\{V=\tau\left(h_{1}\right), I=\tau\left(h_{2}\right), H=\tau\left(h_{3}\right)\right\}$ of $E$, where $\left\{h_{i}\right\}_{i=1,2,3}$ is the standard basis of $A^{1 \times 3}$, satisfying the $B$-linear relation $P\left(\begin{array}{lll}V \quad I \quad H & T\end{array}\right)^{T}=0$, can be expressed as $\left(\begin{array}{lll}V & I & H\end{array}\right)^{T}=Q \rho(T)$. For more details, see [13, 33].

Let us denote by $Q_{1}$ the first two entries of $Q$

$>Q_{-} 1:=\operatorname{submatrix}(Q, 1 \ldots 2,1 \ldots 1)$;

$$
Q_{1}:=\left[\begin{array}{c}
-\alpha^{2} d t+\alpha d x-R C \\
-\alpha C d t-\alpha G+C d x
\end{array}\right]
$$

and $Q_{2}$ the last one:

$>$ Q_2:=collect (submatrix $(\mathrm{Q}, 3 \ldots 3,1 \ldots 1),\{\mathrm{dx}, \mathrm{dt}\}$, distributed);

$$
Q_{2}:=\left[-G R+(-R C-L G) d t+d x^{2}-\alpha^{2} d t^{2}\right]
$$

Theorem 5 then shows that $M \cong B /\left(B Q_{2}\right)$, i.e., $M$ is a cyclic $B$-module.

Since the column vector $\Lambda$ admits the following left-inverse over $B$

$>$ Gamma:=LeftInverse(Lambda_2,B);

$$
\Gamma:=\left[\begin{array}{ll}
0 & C^{-1}
\end{array}\right]
$$

Corollary 5 shows that the matrix $J$ is equivalent to $\operatorname{diag}\left(1, Q_{2}\right)$, i.e., $V J W=\operatorname{diag}\left(1, Q_{2}\right)$, where $V, W \in \mathrm{GL}_{2}(A)$. Let us compute the unimodular matrices $V$ and $W$. We first compute $\operatorname{ker}_{B}\left(. Q_{1}\right)$ :

$>\mathrm{K}:=\operatorname{SyzygyModule}\left(\mathrm{Q}_{-} 1, \mathrm{~B}\right)$;

$$
K:=\left[\begin{array}{ll}
-\alpha C d t-\alpha G+C d x \quad R C+\alpha^{2} d t-\alpha d x
\end{array}\right]
$$

Since $\operatorname{ker}_{B}\left(. Q_{1}\right)=B K$, the $B$-module $\operatorname{ker}_{B}\left(. Q_{1}\right)$ is free of rank 1 . In particular, if we compute a right-inverse $Q_{3}$ of the row vector $K$

$>$ Q_3:=RightInverse $(K, B)$;

$$
Q_{3}:=\left[\begin{array}{l}
\frac{\alpha}{-\alpha^{2} G+R C^{2}} \\
\frac{C}{-\alpha^{2} G+R C^{2}}
\end{array}\right]
$$

then we obtain the following matrix $W=\left(\begin{array}{ll}Q_{3} & Q_{1}\end{array}\right)$

$>\mathrm{W}:=\operatorname{simplify}\left(\operatorname{subs}\left(\operatorname{alpha}{ }^{\wedge} 2=\mathrm{L} * \mathrm{C}, \operatorname{augment}\left(\mathrm{Q}_{-} 3, \mathrm{Q}_{-} 1\right)\right)\right)$;

$$
W:=\left[\begin{array}{cc}
-\frac{\alpha}{C(-R C+L G)} & -L C d t+\alpha d x-R C \\
-(-R C+L G)^{-1} & -\alpha C d t-\alpha G+C d x
\end{array}\right]
$$

and $W \in \mathrm{GL}_{2}(B)$ since:

$$
>\mathrm{W}:=\operatorname{simplify}\left(\operatorname{subs}\left(\operatorname{alpha}^{\wedge} 2=\mathrm{L} * \mathrm{C}, \operatorname{det}(\mathrm{W})\right)\right) \text {; }
$$

Finally, if we define the matrix $X=\left(\begin{array}{ll}J Q_{3} & \Lambda\end{array}\right)$, namely,

$>\quad X:=\operatorname{simplify}\left(\operatorname{subs}\left(\operatorname{alpha}{ }^{\wedge} 2=L * C\right.\right.$, augment $\left(\operatorname{Mult}\left(J, Q_{-} 3, B\right)\right.$, Lambda_2 $\left.\left.)\right)\right)$;

$$
X:=\left[\begin{array}{cc}
-\frac{R C+L C d t+\alpha d x}{C(-R C+L G)} & \alpha \\
-\frac{\alpha C d t+\alpha G+C d x}{C(-R C+L G)} & C
\end{array}\right]
$$

$\mathrm{RR} \mathrm{n}^{\circ} 7214$ 
then its inverse $V$ is defined by

$>\quad \mathrm{V}:=\operatorname{simplify}\left(\operatorname{subs}\left(\operatorname{alpha}{ }^{\wedge} 2=\mathrm{L} * \mathrm{C}, \operatorname{Left} \operatorname{Inverse}(\mathrm{X}, \mathrm{B})\right)\right)$;

$$
V:=\left[\begin{array}{cc}
C & -\alpha \\
\frac{\alpha C d t+\alpha G+C d x}{C(-R C+L G)} & -\frac{R C+L C d t+\alpha d x}{C(-R C+L G)}
\end{array}\right]
$$

i.e., $V \in \mathrm{GL}_{2}(B)$, and we finally have $V J W=\operatorname{diag}\left(1, Q_{2}\right)$ :

$>\operatorname{map}\left(\operatorname{collect}, \operatorname{simplify}\left(\operatorname{subs}\left(\operatorname{alpha}{ }^{\wedge} 2=L * C, \operatorname{Mult}(\mathrm{V}, \mathrm{J}, \mathrm{W}, \mathrm{B})\right)\right),\{\mathrm{dx}, \mathrm{dt}\}\right.$,

$>$ distributed);

$$
\left[\begin{array}{cc}
1 & 0 \\
0 & -G R+(-R C-L G) d t-C d t^{2} L+d x^{2}
\end{array}\right]
$$

Example 23. We consider the conjugate Beltrami equations (16) studied in Examples 6, 12 and 15 . We first introduce the first Weyl algebra $A=A_{2}(\mathbb{Q})=\mathbb{Q}[x, y][d x, d y]$ of differential operators in $d x$ and $d y$ with coefficients in the commutative polynomial ring $\mathbb{Q}[x, y]$ :

$>A:=$ DefineOreAlgebra $(\operatorname{diff}=[\mathrm{dx}, \mathrm{x}], \operatorname{diff}=[\operatorname{dy}, \mathrm{y}], \operatorname{polynom}=[\mathrm{x}, \mathrm{y}], \operatorname{comm}=[\mathrm{a}, \mathrm{b}])$ :

The presentation matrix (16) is defined by:

$>\mathrm{R}:=\operatorname{evalm}([[\mathrm{dx},-\mathrm{x} * \mathrm{dy}],[\mathrm{dy}, \mathrm{x} * \mathrm{dx}]])$;

$$
R:=\left[\begin{array}{cc}
d x & -x d y \\
d y & x d x
\end{array}\right]
$$

Let us introduce the following column vector

$>$ Lambda:=evalm $([[\mathrm{a}],[\mathrm{b}]])$;

$$
\Lambda:=\left[\begin{array}{l}
a \\
b
\end{array}\right]
$$

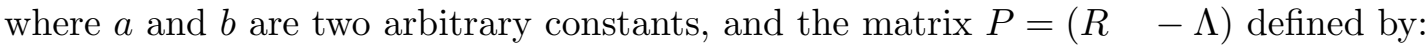

$>\mathrm{P}:=\operatorname{augment}(\mathrm{R},-$ Lambda $)$;

$$
P:=\left[\begin{array}{ccc}
d x & -x d y & -a \\
d y & x d x & -b
\end{array}\right]
$$

Let us check whether or not the matrix $P$ admits a right-inverse over $A$ :

$>$ RightInverse $(\mathrm{P}, \mathrm{A})$;

$$
\left[\begin{array}{cc}
\frac{x(a x d x+x d y b+a)}{a} & -\frac{x(a x d x+x d y b+a)}{b} \\
-\frac{a d y x-2 b-d x b x}{a} & \frac{a d y x-2 b-d x b x}{b} \\
\frac{x\left(x d x^{2}+3 d x+x d y^{2}\right)}{a} & -\frac{1+x^{2} d x^{2}+3 x d x+x^{2} d y^{2}}{b}
\end{array}\right]
$$

We obtain that $P$ admits the previous right-inverse whenever $a \neq 0$ and $b \neq 0$, which shows that $P$ generically admits a right-inverse over $A$. We shall suppose that $a \neq 0$ and $b \neq 0$ in what follows. Then, the left $A$-module $E=A^{1 \times 3} /\left(A^{1 \times 2} P\right)$ is a stably free of rank 1 .

Let us compute minimal parametrizations of $E$, namely, matrices $L_{i} \in A^{3}$ such that the left $A$-modules $N_{i}=A /\left(A^{1 \times 3} L_{i}\right)$ are torsion and $\operatorname{ker}_{A}\left(. L_{i}\right)=A^{1 \times 2} R$, i.e., $E \cong A^{1 \times 3} L_{i}$. 
$>\mathrm{L}:=\operatorname{map}(\operatorname{collect}, \operatorname{MinimalParametrizations}(\mathrm{P}, \mathrm{A}),\{\mathrm{x}, \mathrm{y}, \mathrm{dx}, \mathrm{dy}\}$, distributed):

$>\operatorname{nops}(\mathrm{L})$;

The Oremodules command MinimalParametrizations returns 2 minimal parametrizations. The first one is

$>$ L $[1]$;

$$
\left[\begin{array}{c}
a x d y^{2} b-a d x^{2} b x+a d x b+d y b^{2}+\left(a^{2}-b^{2}\right) d y x d x \\
-a^{2} d y^{2}+2 a d y d x b-d x^{2} b^{2} \\
a d x^{2} x d y+a d y d x+a d y^{3} x-d x^{3} b x+d y^{2} b-d x d y^{2} b x
\end{array}\right]
$$

and the second one is:

$$
\left.\begin{array}{l}
>\mathrm{L} \text { [2] ; } \\
-b a^{2}-x d y a^{3}+d x b a^{2} x-a\left(a^{2}+b^{2}\right) x^{2} d y d x-b\left(a^{2}+b^{2}\right) x^{2} d y^{2} \\
a\left(a^{2}+b^{2}\right) x d y^{2}+d x b^{2} a-b\left(3 a^{2}+2 b^{2}\right) d y-b\left(a^{2}+b^{2}\right) d y x d x \\
a x d y^{2} b+a d x^{2} b x-a^{2} d y-\left(a^{2}+b^{2}\right) d x^{2} x^{2} d y-\left(a^{2}+b^{2}\right) x^{2} d y^{3}-3\left(a^{2}+b^{2}\right) d y x d x
\end{array}\right]
$$

Let us check whether or not they are injective, i.e., whether or not they admit a left-inverse:

$>\operatorname{map}($ LeftInverse, $\mathrm{L}, \mathrm{A})$;

\section{[[], []]}

None of them is injective. The left $A$-module $N_{1}=A /\left(A^{1 \times 3} L_{1}\right)$ is then defined by

$>$ J_1:=map (collect, Exti (Involution(Min [1] , A) , A, 1), $\{\mathrm{dx}, \mathrm{dy}, \mathrm{x}, \mathrm{y}\}$, distributed);

$$
J_{1}:=\left[\left[\begin{array}{c}
d x^{2} b^{2}-2 a d y d x b+a^{2} d y^{2} \\
\left(-b^{2} a-a^{3}\right) x d y^{2}-d x b^{2} a-d y b^{3}+\left(b a^{2}+b^{3}\right) x d y d x
\end{array}\right],[1], S U R J(1)\right]
$$

i.e., the two entries of the first matrix $J_{1}[1]$ of $J_{1}$ annihilate the generator $\sigma_{1}(1)$ of $N_{1}$, where $\sigma_{1}(1)$ denotes the residue class of the standard basis 1 of $A$ in $N_{1}$.

$>$ J_2:=map (collect,Exti (Involution(Min [2], A), A, 1), $\{\mathrm{dx}, \mathrm{dy}, \mathrm{x}, \mathrm{y}\}$, distributed);

$J_{2}:=\left[\left[\begin{array}{c}-d x b^{2} a+\left(2 b^{3}+3 b a^{2}\right) d y+\left(b a^{2}+b^{3}\right) x d y d x+\left(-b^{2} a-a^{3}\right) x d y^{2} \\ a^{2} b^{2}+\left(-2 a^{3} b-2 a b^{3}\right) x d y+\left(2 a^{2} b^{2}+a^{4}+b^{4}\right) x^{2} d y^{2}\end{array}\right],[1]\right.$, SURJ (1)]

Similarly, the two entries of the first matrix $J_{2}[1]$ of $J_{2}$ annihilates the generator $\sigma_{2}(1)$ of $N_{2}$, where $\sigma_{2}(1)$ denotes the residue class of 1 in the left $A$-module $N_{2}=A /\left(A^{1 \times 3} L_{2}\right)$, i.e., $\sigma_{2}(1)$ satisfies $d_{i} \sigma_{2}(1)=0$, for $i=1,2$, where $d_{1} \in A$ is defined by

$>\mathrm{N} 2[1][1,1]$;

$$
-d x b^{2} a+\left(2 b^{3}+3 b a^{2}\right) d y+\left(b a^{2}+b^{3}\right) x d y d x+\left(-b^{2} a-a^{3}\right) x d y^{2}
$$

and $d_{2}$ is defined by:

$>\mathrm{N} 2[1][2,1]$;

$$
a^{2} b^{2}+\left(-2 a^{3} b-2 a b^{3}\right) x d y+\left(2 a^{2} b^{2}+a^{4}+b^{4}\right) x^{2} d y^{2}
$$


Since the two entries of $J_{1}[1]$ do not contain constant terms, they cannot be equal to nonzero constants for particular values of the constants $a$ and $b$. The same comment holds for $d_{1}$. However, the coefficients of $d_{2}$ in $d x$ and $d y$ are:

$$
\begin{gathered}
>1:=[\operatorname{coeffs}(\%,\{\mathrm{dx}, \operatorname{dy}\})]: \operatorname{coefs}:=\operatorname{map}(\text { factor, map }(\operatorname{coeffs}, 1, \mathrm{x})) ; \\
\text { coefs }:=\left[a^{2} b^{2},\left(a^{2}+b^{2}\right)^{2},-2 b a\left(a^{2}+b^{2}\right)\right]
\end{gathered}
$$

Let us find $a$ and $b$ such that $d_{2}$ becomes the non-zero constant -1 :

$$
\begin{aligned}
& >\text { Eqs }:=\{\operatorname{coefs}[1]=-1, \operatorname{seq}(\operatorname{coefs}[\mathrm{i}]=0, \mathrm{i}=2 \ldots \operatorname{nops}(\operatorname{coefs}))\} ; \\
& \text { Eqs }:=\left\{\left(a^{2}+b^{2}\right)^{2}=0, a^{2} b^{2}=-1,-2 b a\left(a^{2}+b^{2}\right)=0\right\} \\
& >\text { Sols }:=\operatorname{solve}(\text { Eqs },\{\mathrm{a}, \mathrm{b}\}) ; \\
& \text { Sols }:=\left\{a=\operatorname{RootOf}\left(-Z^{2}+1\right), b=1\right\},\left\{a=\operatorname{RootOf}\left(-Z^{2}+1\right), b=-1\right\}, \\
& \left\{a=1, b=\operatorname{RootOf}\left(Z^{2}+1\right)\right\},\left\{a=-1, b=\operatorname{RootOf}\left(Z^{2}+1\right)\right\}
\end{aligned}
$$

For instance, if we take $a=1$ and $b=i$, then the coefficients of $d_{2}$ become:

$>\operatorname{subs}(\{a=1, b=I\}, \operatorname{coefs}) ;$

$$
[-1,0,0]
$$

Hence, let us consider the new ring $B=\mathbb{Q}[i] /\left(i^{2}+1\right)[x, y][d x, d y]$ of differential operators in $d x$ and $d y$ with coefficients in the field $\mathbb{Q}(i)=\mathbb{Q}[i] /\left(i^{2}+1\right)$ :

$>B:=$ DefineOreAlgebra $(\operatorname{diff}=[\mathrm{dx}, \mathrm{x}], \operatorname{diff}=[\mathrm{dy}, \mathrm{y}], \operatorname{polynom}=[\mathrm{x}, \mathrm{y}], \mathrm{comm}=[\mathrm{i}, \mathrm{a}, \mathrm{b}]$,

$>$ alg_relations $\left.=\left[i^{\wedge} 2=-1\right]\right)$ :

The column vector $\Lambda$ is then

$>\operatorname{Lambda} \_2:=\operatorname{subs}(\{a=1, b=i\}, \operatorname{evalm}($ Lambda $))$;

$$
\Lambda_{2}:=\left[\begin{array}{l}
1 \\
i
\end{array}\right]
$$

and the matrix $P$ becomes:

$>$ P_2: $=\operatorname{simplify}\left(\operatorname{subs}\left(\left\{i^{\wedge} 2=-1, i^{\wedge} 3=-i\right\}, \operatorname{subs}(\{a=1, b=i\}, \operatorname{evalm}(P))\right)\right)$;

$$
P_{2}:=\left[\begin{array}{ccc}
d x & -x d y & -1 \\
d y & x d x & -i
\end{array}\right]
$$

Substituting $a=1$ and $b=i$ into $L_{2}$, we obtain the matrix $Q$ defined by:

$>Q:=\operatorname{simplify}\left(\operatorname{subs}\left(\left\{i^{\wedge} 2=-1, i^{\wedge} 3=-i\right\}, \operatorname{subs}(\{a=1, b=i\}, \operatorname{evalm}(L[2]))\right)\right)$;

$$
Q:=\left[\begin{array}{c}
-i-x d y+d x i x \\
-d x-d y i \\
x d y^{2} i+d x^{2} i x-d y
\end{array}\right]
$$

We can check that the last matrix defines a minimal parametrization of $B^{1 \times 3} /\left(B^{1 \times 2} P_{2}\right)$ :

$>$ MinimalParametrizations(P_2,B); 


$$
\left.\left[\begin{array}{c}
-d x i x+i+x d y \\
d x+d y i \\
-d x^{2} i x+d y-x d y^{2} i
\end{array}\right]\right]
$$

Moreover, the minimal parametrization $Q$ admits a left-inverse over $B$ defined by:

$>\mathrm{T}:=$ Left Inverse $(\mathrm{Q}, \mathrm{B})$;

$$
T:=\left[\begin{array}{lll}
-i^{-1} & -x & 0
\end{array}\right]
$$

Hence, the left $B$-module $F=B^{1 \times 3} /\left(B^{1 \times 2} P_{2}\right)$ is free of rank 1 and Theorem 5 shows that $F$ is isomorphic to the cyclic left $B$-module $B /\left(B Q_{2}\right)$, where $Q_{2}$ is defined by:

$>$ Q_2:=submatrix $(Q, 3 \ldots 3,1 \ldots 1)$;

$$
Q_{2}:=\left[x d y^{2} i+d x^{2} i x-d y\right]
$$

Moreover, the column vector $\Gamma$ admits the following left-inverse $\Gamma$ over $B$ :

$>$ Gamma:=LeftInverse(Lambda_2,B);

$$
\Gamma:=\left[\begin{array}{ll}
0 & i^{-1}
\end{array}\right]
$$

If we denote by $Q_{1} \in B^{2}$ defined by the first two components of $Q$

$>Q_{-} 1:=\operatorname{submatrix}(Q, 1 \ldots 2,1 \ldots 1)$;

$$
Q_{1}:=\left[\begin{array}{c}
-i-x d y+d x i x \\
-d x-d y i
\end{array}\right]
$$

then Corollary 5 shows that $\operatorname{ker}_{B}\left(. Q_{1}\right)$ is a stably free left $B$-module of rank 1 . Moreover, we have $\operatorname{ker}_{B}\left(. Q_{1}\right)=B K$, where the matrix $K$ is defined by

$>\mathrm{K}:=$ SyzygyModule $\left(\mathrm{Q}_{-} 1, \mathrm{~B}\right)$;

$$
K:=\left[\begin{array}{ll}
-d x i+d y & d y i x+x d x
\end{array}\right]
$$

i.e., $\operatorname{ker}_{B}\left(. Q_{1}\right)$ is a free left $B$-module of rank 1. Corollary 5 then shows that the matrices $R$ and $\operatorname{diag}\left(1, Q_{2}\right)$ are equivalent, where $Q_{2}=i x\left(d x^{2}+d y^{2}\right)-d y$. Let us compute two matrices $V, W \in \mathrm{GL}_{2}(B)$ such that $V R W=\operatorname{diag}\left(1, Q_{2}\right)$.

The right-inverse $Q_{3}$ of $K$ over $B$ defined

$>$ Q_3:=RightInverse (K,B);

$$
Q_{3}:=\left[\begin{array}{r}
-\frac{x}{i} \\
-1
\end{array}\right]
$$

is such that the following matrix $W=\left(\begin{array}{ll}Q_{3} & Q_{1}\end{array}\right)$ defined by

$>\mathrm{W}:=$ augment (Q_3, Q_1) ;

$$
W:=\left[\begin{array}{cc}
-\frac{x}{i} & -i-x d y+d x i x \\
-1 & -d x-d y i
\end{array}\right]
$$

is unimodular, i.e., $W \in \mathrm{GL}_{2}(B)$ :

$>$ W_inv: =LeftInverse (W, B); 


$$
W_{-i n v}:=\left[\begin{array}{cc}
-d x i+d y & d y i x+x d x \\
i & -x
\end{array}\right]
$$

Moreover, the matrix $X=\left(\begin{array}{ll}R Q_{3} & \Lambda\end{array}\right)$ defined by

$>\quad \mathrm{X}:=\operatorname{augment}\left(\operatorname{Mult}\left(\mathrm{R}, \mathrm{Q} \_3, \mathrm{~B}\right)\right.$, Lambda_2) ;

$$
X:=\left[\begin{array}{cc}
\frac{-x d x-1+d y i x}{i} & 1 \\
-\frac{x(d y+d x i)}{i} & i
\end{array}\right]
$$

i.e., after simplifications, by

$>\operatorname{map}(\operatorname{expand}, \operatorname{subs}(\mathrm{i}=\mathrm{I}, \operatorname{evalm}(\mathrm{X})))$;

$$
\left[\begin{array}{cc}
i x d x+i+x d y & 1 \\
i d y x-x d x & i
\end{array}\right]
$$

is also unimodular, i.e., $X \in \mathrm{GL}_{2}(B)$, and its inverse $V=X^{-1}$ is defined by

$>\quad \mathrm{V}:=$ LeftInverse $(\mathrm{X}, \mathrm{B})$;

$$
V:=\left[\begin{array}{cc}
i^{-1} & 1 \\
-x d x+d y i x & -i-x d y-d x i x
\end{array}\right]
$$

or, equivalently, after simplifications, by

$>\operatorname{map}(\operatorname{expand}, \operatorname{subs}(i=I, \operatorname{evalm}(V)))$;

$$
\left[\begin{array}{cc}
-i & 1 \\
i d y x-x d x & -i-x d y-i x d x
\end{array}\right]
$$

Finally, we obtain that $V R W=\operatorname{diag}\left(1, Q_{2}\right)$ :

$>\operatorname{map}(\operatorname{collect}, \operatorname{subs}(i=I, \operatorname{Mult}(V, R, W, B)), x)$;

$$
\left[\begin{array}{cc}
1 & 0 \\
0 & i x\left(d x^{2}+d y^{2}\right)-d y
\end{array}\right]
$$

Finally, we refer the reader to SERRE webpages ([1]) for a library of examples coming from mathematical systems theory, control theory, engineering sciences and mathematical physics.

\section{References}

[1] R. Baer, Erweiterung von Gruppen und ihren Isomorphismen. Mathematische Zeitschrift 38 (1934), 375-416.

[2] N. K. Bose, Multidimensional Systems Theory and Applications. $2^{\text {nd }}$ edition, Kluwer Academic Press, 2003.

[3] M. S. Boudellioua, Equivalence to Smith form over a multivariate polynomial ring. Proceedings of the $4^{\text {th }}$ International Workshop on Multidimensional Systems, 2005, 259-262.

[4] M. S. Boudellioua, A. Quadrat, Reduction of linear systems based on Serre's theorem. Proceedings of MTNS 2008, Virginia (USA) (28/07-01/08/08). 
[5] F. Chyzak, B. Salvy, "Non-commutative elimination in Ore algebras proves multivariate identities", Journal of Symbolic Computation, 26 (1998), 187-227.

[6] F. Chyzak, A. Quadrat, D. Robertz, Effective algorithms for parametrizing linear control systems over Ore algebras. Appl. Algebra Engrg. Comm. Comput. 16 (2005), 319-376.

[7] F. Chyzak, A. Quadrat, D. Robertz, OreModules: A symbolic package for the study of multidimensional linear systems. Applications of Time-Delay Systems, J. Chiasson and J. J. Loiseau (Eds.), LNCIS 352, Springer, 2007, 233-264, OreModules project: http:// wwwb.math.rwth-aachen.de/OreModules.

[8] T. Cluzeau, A. Quadrat, Factoring and decomposing a class of linear functional systems. Linear Algebra and Its Applications 428 (2008), 324-381.

[9] T. Cluzeau, A. Quadrat, OrEMorPhISMs: A homological algebraic package for factoring and decomposing linear functional systems. Topics in Time-Delay Systems: Analysis, Algorithms and Control, J.-J. Loiseau, W. Michiels, S.-I. Niculescu and R. Sipahi (Eds.), LNCIS 388, Springer, 2009, 179-196, OREMORPHISMS project: http://www-sop.inria.fr/members/ Alban.Quadrat/OreMorphisms/index.html.

[10] T. Cluzeau, A. Quadrat, Serre's reduction of linear systems of partial differential equations based on holonomy. Submitted to MTNS 2010, Budapest.

[11] T. Cluzeau, A. Quadrat, SeRRE project. In development.

[12] D. Eisenbud, Commutative Algebra with a View Toward Algebraic Geometry, Graduate Texts in Mathematics 150, Springer, 1995.

[13] A. Fabiańska, A. Quadrat, Applications of the Quillen-Suslin theorem in multidimensional systems theory. Gröbner Bases in Control Theory and Signal Processing, H. Park and G. Regensburger (Eds.), Radon Series on Computation and Applied Mathematics 3, de Gruyter publisher, 2007, 23-106, QuillenSuslin project: http://wwwb.math.rwth-aachen.de/ QuillenSuslin

[14] M. G. Frost, C. Storey, Equivalence of a matrix over $R[s, z]$ with its Smith form. Internat. J. Control 28 (1978), 665-671.

[15] M. G. Frost, C. Storey, Equivalence of a matrix over $R[s, z]$ : A counter-example. Internat. J. Control 34 (1981), 1225-1226.

[16] M. G. Frost, M. S. Boudellioua, Some further results concerning matrices with elements in a polynomial ring. Internat. J. Control 43 (1986), 1534-1555.

[17] T. Kailath, Linear Systems. Englewood Cliffs: Prentice-Hall, 1980.

[18] V. Kolmanovskii, V. Nosov, Stability of Functional Differential Equations. Academic Press, 1986.

[19] T. Y. Lam, Lectures on Modules and Rings. Graduate Texts in Mathematics, 189, Springer, 1999.

[20] E. B. Lee, S. H. Żak, Smith forms over $R\left[z_{1}, z_{2}\right]$. IEEE Trans. Automatic Control 28 (1983), $115-118$. 
[21] V. Levandovskyy, E. Zerz, Obstructions to genericity in the study of parametric problems in control theory. Gröbner Bases in Control Theory and Signal Processing, H. Park and G. Regensburger (Eds.), Radon Series on Computation and Applied Mathematics 3, de Gruyter publisher, 2007, 127-149, SINGULAR project: http://www.singular.uni-kl.de/.

[22] B. Malgrange, Systèmes à coefficients constants. Séminaire Bourbaki, 1962/63, 1-11.

[23] A. Manitius, Feedback controllers for a wind tunnel model involving a delay: analytical design and numerical simulations. IEEE Trans. Autom. Contr. 29 (1984), 1058-1068.

[24] J. C. McConnell, J. C. Robson, Noncommutative Noetherian Rings. American Mathematical Society, 2000.

[25] H. Mounier, J. Rudolph, M. Fliess, P. Rouchon, Tracking control of a vibrating string with an interior mass viewed as delay system. ESAIM Control Optim. Calc. Var. 3 (1998), 315321.

[26] H. Mounier, P. Rouchon, J. Rudolph, Some examples of linear systems with delays. European Journal of Automation 31 (1997), 911-925.

[27] U. Oberst, Multidimensional constant linear systems. Acta Appl. Math. 20 (1990), 1-175.

[28] J.-F. Pommaret, A. Quadrat, Algebraic analysis of linear multidimensional control systems. IMA J. Math. Control Inform. 16 (1999), 275-297.

[29] J.-F. Pommaret, A. Quadrat, Formal elimination for multidimensional systems and applications to control theory. Math. Control, Signal and Systems 13, (2000), 193-215.

[30] A. Quadrat, The fractional representation approach to synthesis problems: An algebraic analysis viewpoint. Part I: (Weakly) doubly coprime factorizations. SIAM J. Control \& Optimization, 42 (2003), 266-299.

[31] A. Quadrat, Purity filtration of 2-dimensional linear functional systems. Submitted to MTNS 2010, Budapest.

[32] A. Quadrat, Purity filtration of of linear systems of partial differential equations in two independent variables, INRIA report, to appear, 2010.

[33] A. Quadrat, D. Robertz, Computation of bases of free modules over the Weyl algebras. J. Symbolic Comput. 42 (2007), 1113-1141, STAFFord project: http://wwwb.math. rwth-aachen.de/OreModules.

[34] A. Quadrat, D. Robertz, On the Baer extension problem for multidimensional linear systems, INRIA report 6307, 2007, http://hal.inria.fr/inria-00175272.

[35] A. Quadrat, D. Robertz, Baer's extension problem for multidimensional linear systems. Proceedings of MTNS 2008, Virginia (USA), (28/07-01/08/08).

[36] A. Quadrat, D. Robertz, Controllability and differential flatness of linear analytic ordinary differential systems. Submitted to MTNS 2010, Budapest.

[37] H. H. Rosenbrock, State Space and Multivariable Theory. Wiley-Interscience, 1970.

[38] J. J. Rotman, An Introduction to Homological Algebra. $2^{\text {nd }}$ edition, Springer, 2009. 
[39] J.-P. Serre, Sur les modules projectifs. Séminaire Dubreil-Pisot, vol. 2, 1960/1961, in Oeuvres, Collected Papers, Vol. II 1960-1971, Springer, 1986, 23-34.

[40] E. Zerz, Topics in Multidimensional Linear Systems Theory, Lecture Notes in Control and Information Sciences 256, Springer, 2000. 
Centre de recherche INRIA Sophia Antipolis - Méditerranée 2004, route des Lucioles - BP 93 - 06902 Sophia Antipolis Cedex (France)

Centre de recherche INRIA Bordeaux - Sud Ouest : Domaine Universitaire - 351, cours de la Libération - 33405 Talence Cedex Centre de recherche INRIA Grenoble - Rhône-Alpes : 655, avenue de l'Europe - 38334 Montbonnot Saint-Ismier

Centre de recherche INRIA Lille - Nord Europe : Parc Scientifique de la Haute Borne - 40, avenue Halley - 59650 Villeneuve d'Ascq Centre de recherche INRIA Nancy - Grand Est : LORIA, Technopôle de Nancy-Brabois - Campus scientifique 615, rue du Jardin Botanique - BP 101 - 54602 Villers-lès-Nancy Cedex

Centre de recherche INRIA Paris - Rocquencourt : Domaine de Voluceau - Rocquencourt - BP 105 - 78153 Le Chesnay Cedex

Centre de recherche INRIA Rennes - Bretagne Atlantique : IRISA, Campus universitaire de Beaulieu - 35042 Rennes Cedex Centre de recherche INRIA Saclay - Île-de-France : Parc Orsay Université - ZAC des Vignes : 4, rue Jacques Monod - 91893 Orsay Cedex 